\title{
PENELLOPE: The ESO data legacy program to complement the Hubble UV Legacy Library of Young Stars (ULLYSES)
}

\section{Survey presentation and accretion properties of Orion OB1 and $\sigma$-Orionis ${ }^{\star}$}

\author{
C. F. Manara ${ }^{1}$, A. Frasca ${ }^{2}$, L. Venuti ${ }^{3}$, M. Siwak ${ }^{4}$, G. J. Herczeg ${ }^{5}$, N. Calvet $^{6}$, J. Hernandez ${ }^{7}$, Ł. Tychoniec ${ }^{1}$,
} M. Gangi ${ }^{8}$, J. M. Alcalá ${ }^{9}$, H. M. J. Boffin ${ }^{1}$, B. Nisini ${ }^{8}$, M. Robberto ${ }^{10}$, C. Briceno ${ }^{11}$, J. Campbell-White ${ }^{12}$, A. Sicilia-Aguilar ${ }^{12}$, P. McGinnis ${ }^{13}$, D. Fedele ${ }^{14,15}$, Á. Kóspál ${ }^{4,16,17}$, P. Ábrahám ${ }^{4,17}$, J. Alonso-Santiago ${ }^{2}$, S. Antoniucci ${ }^{8}$, N. Arulanantham ${ }^{10}, \mathrm{~F}$. Bacciotti ${ }^{15}$, A. Banzatti ${ }^{18}$, G. Beccari ${ }^{1}$, M. Benisty ${ }^{19}$, K. Biazzo $^{8}$, J. Bouvier ${ }^{19}$, S. Cabrit ${ }^{20}$, A. Caratti o Garatti ${ }^{13,21}$, D. Coffey ${ }^{21,13}$, E. Covino ${ }^{9}$, C. Dougados ${ }^{19}$, J. Eislöffel ${ }^{22}$, B. Ercolano ${ }^{23}$, C. C. Espaillat ${ }^{24}$, J. Erkal ${ }^{21,1}$, S. Facchini ${ }^{1}$, M. Fang ${ }^{25}$, E. Fiorellino ${ }^{8,4}$, W. J. Fischer ${ }^{10}$, K. France ${ }^{26}$, J. F. Gameiro ${ }^{27}$, R. Garcia Lopez ${ }^{21,13}$, T. Giannini ${ }^{8}$, C. Ginski ${ }^{28}$, K. Grankin ${ }^{29}$, H. M. Günther ${ }^{30}$, L. Hartmann ${ }^{6}$, L. A. Hillenbrand ${ }^{31}$,

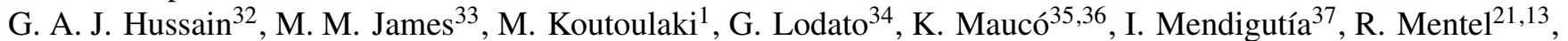
A. Miotello ${ }^{1}$, R. D. Oudmaijer ${ }^{38}$, E. Rigliaco ${ }^{39}$, G. P. Rosotti ${ }^{40,41}$, E. Sanchis ${ }^{1}$, P. C. Schneider ${ }^{42}$, L. Spina ${ }^{39}$, B. Stelzer ${ }^{43,44}$, L. Testi ${ }^{1,15}$, T. Thanathibodee ${ }^{6}$, J. S. Vink ${ }^{45}$, F. M. Walter ${ }^{46}$, J. P. Williams ${ }^{33}$, and G. Zsidi ${ }^{4,17}$

(Affiliations can be found after the references)

Received 23 February 2021 / Accepted 22 March 2021

\section{ABSTRACT}

\begin{abstract}
The evolution of young stars and disks is driven by the interplay of several processes, notably the accretion and ejection of material. These processes, critical to correctly describe the conditions of planet formation, are best probed spectroscopically. Between 2020 and 2022 , about 500 orbits of the Hubble Space Telescope (HST) are being devoted in to the ULLYSES public survey of about 70 low-mass $\left(M_{\star} \leq 2 M_{\odot}\right)$ young (age $<10 \mathrm{Myr}$ ) stars at UV wavelengths. Here, we present the PENELLOPE Large Program carried out with the ESO Very Large Telescope (VLT) with the aim of acquiring, contemporaneously to the HST, optical ESPRESSO/UVES high-resolution spectra for the purpose of investigating the kinematics of the emitting gas, along with UV-to-NIR X-shooter medium-resolution flux-calibrated spectra to provide the fundamental parameters that HST data alone cannot provide, such as extinction and stellar properties. The data obtained by PENELLOPE have no proprietary time and the fully reduced spectra are being made available to the whole community. Here, we describe the data and the first scientific analysis of the accretion properties for the sample of 13 targets located in the Orion OB1 association and in the $\sigma$-Orionis cluster, observed in November-December 2020. We find that the accretion rates are in line with those observed previously in similarly young star-forming regions, with a variability on a timescale of days $(\lesssim 3)$. The comparison of the fits to the continuum excess emission obtained with a slab model on the X-shooter spectra and the HST/STIS spectra shows a shortcoming in the X-shooter estimates of $\lesssim 10 \%$, which is well within the assumed uncertainty. Its origin can be either due to an erroneous UV extinction curve or to the simplicity of the modeling and, thus, this question will form the basis of the investigation undertaken over the course of the PENELLOPE program. The combined ULLYSES and PENELLOPE data will be key in attaining a better understanding of the accretion and ejection mechanisms in young stars.
\end{abstract}

Key words. accretion, accretion disks - protoplanetary disks - stars: pre-main sequence - stars: variables: T Tauri, Herbig Ae/Be

\section{Introduction}

The formation and evolution of stars and planets are processes that are intimately connected and our knowledge of the properties of young stars and their circumstellar disks are key to understanding planet formation (e.g., Morbidelli \& Raymond 2016). The early evolution of stars and disks is regulated by the interplay of several mechanisms, in particular, accretion onto the star (see reviews by Bouvier et al. 2007; Hartmann et al. 2016) and ejection of matter from the disks through winds and outflows (see reviews by e.g., Frank et al. 2014; Ercolano \& Pascucci 2017), as well as processes that are attributed to the effects of the local environment (e.g., Fischer et al. 2017; Winter et al. 2018). These processes are driven by the transfer of angular momen-

* Based on observations collected at the European Southern Observatory under ESO programme 106.20Z8. tum in the disk (e.g., Lynden-Bell \& Pringle 1974; Pringle 1981; Bai 2016; Pudritz \& Ray 2019). Thus, the study of accretion and ejection it is fundamental in order to consistently explain how the initial angular momentum is distributed and how disks are dispersed (e.g., Alexander et al. 2014). Both of these characteristics are necessary for the explanation of why planetary systems are formed so differently from each other.

The task, however, is not a simple one. It is only by observing large samples of young stars and probing wide ranges of age and mass that we can gain access to a sufficient set of statistics to aid in the understanding of both processes. Spectroscopic surveys of young stars in different star-forming regions have shown that the mass accretion rates onto the central star $\left(\dot{M}_{\text {acc }}\right)$ slowly decrease with isochronal ages, possibly in accordance with viscous evolution models (e.g., Hartmann et al. 1998; Sicilia-Aguilar et al. 2010; Antoniucci et al. 2014), although high accretion rates are still observed at ages of $>5-10 \mathrm{Myr}$ 
(e.g., Ingleby et al. 2014; Frasca et al. 2015; Rugel et al. 2018; Venuti et al. 2019; Manara et al. 2020). The empirical measurement of the steep dependence of $\dot{M}_{\text {acc }}$ on the stellar mass $\left(M_{\star}\right)$ (Hillenbrand et al. 1992; Muzerolle et al. 2003; Calvet et al. 2004; Mohanty et al. 2005; Natta et al. 2006; Manara et al. 2012, 2016a, 2017a; Alcalá et al. 2014, 2017; Venuti et al. 2014) may be the consequence of a single dominant phenomenon or a mix of many, such as, initial conditions followed by viscous evolution (e.g., Alexander et al. 2006; Dullemond et al. 2006), an imprint of internal photoevaporation (e.g., Clarke \& Pringle 2006; Ercolano et al. 2014), different accretion regimes at different stellar masses (e.g., Mohanty et al. 2005; Hartmann et al. 2006), environmental effects (e.g., Padoan et al. 2005), or self-gravity in the disk (e.g., Vorobyov \& Basu 2009; DeSouza \& Basu 2017). Recently, the connection of measurements of $\dot{M}_{\text {acc }}$ from spectroscopy and of disk masses with millimeter interferometry, mainly with the Atacama Large Millimeter/submillimeter Array (ALMA), has also revealed the presence of a correlation between these two quantities (e.g., Manara et al. 2016b; Mulders et al. 2017), which is possibly in line with expectations drawn from viscous evolution (e.g., Jones et al. 2012; Lodato et al. 2017; Rosotti et al. 2017), but this also reveals tensions with respect to this simplified description of disk evolution (e.g., Mulders et al. 2017; Manara et al. 2020). These tensions may be relieved by introducing complications to a simplistic model, including external or internal photoevaporation (e.g., Rosotti et al. 2017; Sellek et al. 2020a; Somigliana et al. 2020), or dust evolution (Sellek et al. 2020b).

Winds from protoplanetary disks affect the disk evolution by carrying away angular momentum and perhaps even driving accretion (Ferreira et al. 2006; Bai 2016). Studies of forbidden emission lines emitted from high-velocity jets and outflows have shown that typically the mass loss rate is a factor of $\sim 0.1-0.3$ of $\dot{M}_{\text {acc }}$ (e.g., Hartigan et al. 1995; Nisini et al. 2018). On the other hand, thanks to the study of large samples of young stellar objects, it is becoming more evident that the low-velocity component of forbidden lines, tracing slow disk winds (e.g., Natta et al. 2014) is composed of multiple sub-components tracing either internal photoevaporative winds (e.g., Ercolano \& Owen 2016; Ercolano \& Pascucci 2017; Ballabio et al. 2020) or, most probably, magneto-hydrodynamical winds (e.g., Rigliaco et al. 2013; Simon et al. 2016; Ercolano \& Pascucci 2017; McGinnis et al. 2018; Banzatti et al. 2019; Weber et al. 2020). The combination of multiple emission lines observed at high spectral resolution $\left(\Delta v \lesssim 5-10 \mathrm{~km} \mathrm{~s}^{-1}\right)$ allows us to determine the physical conditions in the winds and in the jets (e.g., Giannini et al. 2019) and to constrain the mass loss rates in these winds (e.g., Fang et al. 2018).

A complete view of the accretion and ejection properties is, nonetheless, achievable solely by combining data at different wavelengths from the ultraviolet (UV) to the optical and to the infrared (IR), since each of these trace different processes and regions of the young stars (e.g., Arulanantham et al. 2018; Banzatti et al. 2019).

Finally, this rich phenomenology is characterized by time variability over timescales that can be as short as a few minutes (e.g., Stauffer et al. 2014; Siwak et al. 2018). The variability of the accretion process has been studied both with spectroscopy (e.g., Basri \& Batalha 1990; Johns \& Basri 1995a; Johns \& Basri 1995b; Jayawardhana et al. 2006; Biazzo et al. 2012; Costigan et al. 2014; Sousa et al. 2016) and with photometry (e.g., Cody \& Hillenbrand 2010; Cody et al. 2014; Venuti et al. 2014). The variability of emission lines is observed both in their intensity and morphology, which can be understood via adequate modeling (e.g., Muzerolle et al. 1998, 2001; Kurosawa et al. 2006).

About 500 orbits of the Hubble Space Telescope (HST) are being devoted to the study of about 70 young low-mass stars in the Director's Discretionary Time ULLYSES program (Roman-Duval et al. 2020). This program is aimed at obtaining low- and medium-resolution spectra of young stars covering the wavelength range from $\sim 140 \mathrm{~nm}$ to $\sim 1 \mu \mathrm{m}$, hence providing an unprecedented view of accretion and ejection tracers at ultraviolet wavelengths. Inspired by this initiative, we proposed (and were granted) a $\sim 250 \mathrm{~h}$ Large Program on the ESO VLT that is aimed at obtaining complementary data to all the targets of the ULLYSES program. This program, named PENELLOPE in order to underline the complementarity to ULLYSES and to match its spelling flaw, is a public (no proprietary time) community-driven effort. PENELLOPE uniquely provides optical high-resolution (even at $R>100000$ ) and medium-resolution flux-calibrated optical and infrared spectra (up to $2.5 \mu \mathrm{m}$ ) at $R>10000$, providing access to the following information that is otherwise not obtainable with the spectra provided by ULLYSES: main stellar properties (luminosity, temperature, gravity, rotational velocity, mass, age), accurate interstellar extinction, and veiling; kinematics and geometry of the accretion process; kinematics and physical properties of disk winds and jets; properties of the hot molecular content of the disk. In this way, the marriage of PENELLOPE and ULLYSES will provide the whole community with a unique and contemporaneous UV, optical, and near-infrared dataset to study the stellar, accretion, and wind and outflow properties of a significant sample of objects spanning a wide range of disk ages $(\sim 1-10 \mathrm{Myr})$ and evolutionary stages, including full and transitional disks, and Class III disk-less targets.

In this work, we present the observing strategy and data reduction and analysis process of the VLT/PENELLOPE program (Sect. 2) and use the sample of targets in the Orion region (see Sect. 3) as a first application. We derive the stellar and accretion properties, as well as the photospheric parameters, the observed variability in key emission lines, and compare the VLT and HST spectra in Sect. 4. A detailed description of the emission line profiles, including the possibility to use this information to study outflows and ejection processes is deferred to future works. We then discuss the accretion properties for these targets in the context of current surveys of accretion properties in Sect. 5. Finally, we outline the conclusions of this work in Sect. 6.

\section{PENELLOPE survey strategy}

\subsection{HST ULLYSES program}

HST is dedicating 1000 orbits of Director's Discretionary Time to the ULLYSES program ${ }^{1}$ (PI Roman-Duval; Roman-Duval et al. 2020), which will generate a spectral library of high- and low-mass young stars. In HST cycles 28 and 29 (2020-2022), about half of these orbits are being used to obtain spectra of about 70 low-mass $\left(\sim 0.1-2 M_{\odot}\right)$ young stars with ages from $\sim 1$ to $10 \mathrm{Myr}$.

Four of the low-mass stars are observed multiple times: twelve times over three consecutive rotation periods and again with the same cadence about a year later. Cosmic Origins Spectrograph (COS) spectra will be obtained for these targets from

https://ullyses.stsci.edu/ 
$140 \mathrm{~nm}$ to $180 \mathrm{~nm}$ with medium resolution $(R \sim 17000)$ and from $250 \mathrm{~nm}$ to $310 \mathrm{~nm}$ with low resolution $(R \sim 3000)$. The others, including all the stars discussed in this paper, are observed once, with COS medium-resolution $(R \sim 15000)$ spectroscopy in the far-ultraviolet $(120 \mathrm{~nm}<\lambda<180 \mathrm{~nm})$ and Space Telescope Imaging Spectrograph (STIS) low-resolution $(R<1000)$ spectroscopy in the near-ultraviolet $(160 \mathrm{~nm}<\lambda<320 \mathrm{~nm})$ and optical $(\lambda<1 \mu \mathrm{m})$, all obtained within $\sim 24 \mathrm{~h}$ of each other.

The targets are located in nine nearby star-forming regions, namely Chamaeleon I, Corona Australis, $\epsilon$ Cha, $\eta$ Cha, Lupus, Orion OB1, $\sigma$-Orionis, TW Hydrae, and Taurus. All these starforming regions are accessible by telescopes located in the Southern Hemisphere. This choice was made given that only very few targets in this part of the sky had been observed with COS and STIS to date (e.g., Arulanantham et al. 2018, 2020) and also for the purpose of enabling synergies between HST and the major ground-based optical to near-infrared (e.g., Very Large Telescope, VLT) and (sub-)mm (e.g., Atacama Large Millimetre Array, ALMA) observatories. The HST COS and STIS observations have been coordinated, when possible, with photometric observations by the NASA Transiting Exoplanet Survey Satellite (TESS, Ricker et al. 2015). In addition, a number of groundbased astronomical observatories are ready to monitor the targets during the HST observations with simultaneous multiband photometry. These data, which are to be made publicly available as soon as they are taken, will serve as a benchmark for future studies of UV radiation from young stars.

\subsection{Observing strategy at VLT}

The observational strategy of the VLT/PENELLOPE program is such that the HST/ULLYSES targets are observed contemporaneously to HST with two major observing modes, that is, highresolution spectroscopy $(R>70000)$ with UVES or ESPRESSO (Sect. 2.2.1), and medium-resolution $(R \sim 10000-20000)$ spectroscopy with X-shooter to obtain broad-wavelength, fluxcalibrated spectra (Sect. 2.2.2). Observations are taken in service mode with tight absolute time constraints of about three days on the X-shooter observation, and with a time-linked concatenation of observations for the high-resolution data, where the first epochs has a two-day absolute time window, followed by the next with a relative time window of a minimum time separation of one night and a maximum of two nights. The exact intervals of the observations are updated about two weeks before the observations once the HST time windows have been finalized.

\subsubsection{High-resolution spectra}

High-resolution spectroscopy is performed either with the Ultraviolet and Visual Echelle Spectrograph (UVES, Dekker et al. 2000) or the Echelle SPectrograph for Rocky Exoplanets and Stable Spectroscopic Observations (ESPRESSO, Pepe et al. 2021), depending on the brightness of the targets. The brighter targets, $V<16.5 \mathrm{mag}$, are observed with the instrument ESPRESSO, a fibre-fed (1.0" wide) spectrograph which allows for $R \sim 140000\left(\Delta v \sim 2 \mathrm{~km} \mathrm{~s}^{-1}\right)$ spectra between $380 \mathrm{~nm}$ and $788 \mathrm{~nm}$ to be obtained. ESPRESSO offers great scheduling flexibility, as it can be operated from any of the four Unit Telescopes (UT) of the VLT. Fainter targets, as well as targets that are to be probed when ESPRESSO is not available (see Sect. 3), are observed with the UVES spectrograph, which provides twice lower $R \sim 70000\left(\Delta v \sim 4 \mathrm{~km} \mathrm{~s}^{-1}\right)$ spectral resolution. This instrument, fed through a long slit, has two arms,
$\operatorname{Red}(\lambda \gtrsim 480 \mathrm{~nm})$ and Blue $(\lambda \lesssim 450 \mathrm{~nm})$. The resolution and wavelength range depend on the specific setting. PENELLOPE uses 0.6" wide slits in both arms, leading to a resolving power $R \sim 70000$, centering the dichroics at $580 \mathrm{~nm}$ (red arm) and $390 \mathrm{~nm}$ (blue arm), respectively. This allows us to simultaneously cover the wavelength ranges $\lambda \sim 330-450 \mathrm{~nm}$, $480-680 \mathrm{~nm}$, with a small gap between $575 \mathrm{~nm}$ and $585 \mathrm{~nm}$. The exposure times are set so that the signal-to-noise ratio $(\mathrm{S} / \mathrm{N})$ at $630 \mathrm{~nm}$ is larger than $\sim 50$ in each observation. The UVES slits are typically oriented at position angles $0^{\circ}, 120^{\circ}$, and $180^{\circ}$ in the three epochs (see later), apart from known cases where the target is a visual binary with separation in the range of $\sim 1^{\prime \prime}-8^{\prime \prime}$. In those cases, the slit is aligned to include both components of the system.

\subsubsection{Medium-resolution flux-calibrated spectra}

The medium-resolution broad-wavelength coverage fluxcalibrated spectroscopy is obtained using the X-shooter instrument (Vernet et al. 2011). This long-slit (11") spectrograph provides simultaneous coverage of the region between $\sim 300 \mathrm{~nm}$ and $\sim 2500 \mathrm{~nm}$, divided into three arms, UVB

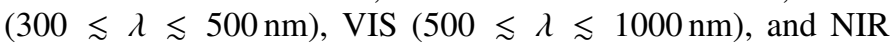
$(1000 \lesssim \lambda \lesssim 2500 \mathrm{~nm})$. Each target is observed first using a set of 5.0"-wide slits in the three arms, leading to a low resolution observation with no slit losses, key to obtain absolute flux calibration of the spectra. Then, using $1.0^{\prime \prime} / 0.4^{\prime \prime} / 0.4^{\prime \prime}$-wide slits for the UVB, VIS, and NIR arms, respectively, high S/N spectra with $R \sim 5400,18400$, and 11600 are obtained in the three arms. The exposure times are set such that the $\mathrm{S} / \mathrm{N}$ at $400 \mathrm{~nm}$ is $23-5$, resulting in a $\mathrm{S} / \mathrm{N}$ in the VIS and NIR arms always $>100$. To mitigate the effects of differential atmospheric dispersion, the slits are always oriented at parallactic angle, apart from known cases where the target is a visual binary with separation $\sim 1^{\prime \prime}-8^{\prime \prime}$. In those cases the slit is also aligned to include both components of the system.

\subsubsection{Sequence of the observations}

In order to measure all the stellar, accretion, and outflow properties while reducing the uncertainties due to the variability of these processes, the timing and number of observations must be closely coordinated with HST. Knowing that each HST observation with the COS and STIS instruments spans $\sim 1-2$ days, per each HST epoch we aim at obtaining:

First, three high-resolution spectra (ESPRESSO or UVES), each one taken on the night before, on the night of the HST observations, and on the following night. These are needed to have a detailed understanding of the line profiles, especially to study accretion and ejection processes, and their short-term variability in order to estimate and correct for any variability of the accretion and ejection processes occurring between the time of the HST and VLT observations.

Second, a combination of narrow-slit (for the resolution) plus wide-slit (for the flux-calibration) medium-resolution $\mathrm{X}$-shooter spectra possibly in the same night, in any case, within \pm 2 days from the HST observation, to provide an accurate measurement across the spectrum of the target brightness at the time of the observation and to study several spectral features from the Balmer continuum to the $K$-band in the infrared.

Any lack of availability of instruments or poor atmospheric conditions may hinder the possibility of executing this strategy. Therefore, these points represent a guideline to be followed as 
much as possible, but which could be altered if needed. In any case, the goal is to obtain three high-resolution spectra and one $\mathrm{X}$-shooter spectrum per each HST target and observation.

\subsection{Contemporaneous photometry}

Photometric data that are close in time to the ULLYSES and PENELLOPE observations presented in this paper were collected from several ground-based observatories with the aim of studying the variability and to provide a reference for absolute flux calibration.

In this work, we make use of data taken at the M. G. Fracastoro station (Serra La Nave, Mt. Etna, $1750 \mathrm{~m}$ a.s.l.) of the Osservatorio Astrofisico di Catania (OACT, Italy) from 25 November to 16 December 2020 . We used the facility imaging camera at the $0.91 \mathrm{~m}$ telescope with a set of broadband Bessel filters $(B, V, R, I, Z)$ as well as two narrow-band filters, $\mathrm{H} \alpha_{9}$ and $\mathrm{H} \alpha_{18}$, centered at $656.8 \mathrm{~nm}$ and at $676.4 \mathrm{~nm}$, whose full widths at half-maximum are 9 and $18 \mathrm{~nm}$, respectively. The index $\mathrm{H} \alpha_{18}-\mathrm{H} \alpha_{9}$ has a weak dependence on the photospheric parameters and is basically a measure of intensity of the $\mathrm{H} \alpha$ emission in units of the continuum that can be converted into $\mathrm{H} \alpha$ equivalent width (Frasca et al. 2018). The $Z$ filter has a transmittance that produces a passband very similar to that of the Sloan $z^{\prime}$ filter when it is multiplied by the responsive quantum efficiency of the adopted CCD. The CCD camera adopts a Kodak KAF $1001 \mathrm{E}^{2} 1 \mathrm{k} \times 1 \mathrm{k}$ chip that, with a focal reducer, covers a field of view of about $11.5 \times 11.5$ arcmin.

The broadband $B V R I$ photometry was calibrated using the following procedure. The stars in the standard areas GD 71 and SA 98 (Stetson 2000; Landolt 2009) were observed on the nights with the best photometric conditions. These observations were used to calculate, for a number of non-variable stars in the same fields of the targets, the zero points and transformation coefficients to the Johnson-Cousins system. The transformations to the Johnson-Cousins system were then used to derive the standard magnitudes of our targets. The magnitudes of the local comparison stars retrieved from SDSS (Alam et al. 2015) and Pan-STARRS (Chambers et al. 2016) survey catalogs were used to get $z^{\prime}$ magnitudes of our targets. For details about the OACT data and their reduction and the narrow-band $\mathrm{H} \alpha$ photometry, we refer to Frasca et al. (2018).

We also used BVri photometry collected by AAVSOnet ${ }^{3}$, which is a set of robotic telescopes operated by volunteers for the American Association of Variable Star Observers (AAVSO). Stars in the AAVSO Photometric All-Sky Survey (APASS, Henden et al. 2018) were used to calibrate the AAVSO photometric data for our targets. The $r, i$ magnitudes were converted to $R_{\mathrm{C}}, I_{\mathrm{C}}$ using the prescription given by $\mathrm{SDSS}^{4}$.

Some additional photometry was obtained at the Crimean Astrophysical Observatory (CrAO) on the AZT-11 $1.25 \mathrm{~m}$ telescope equipped with a CCD camera (with the ProLine PL23042 detector) and a set of broadband Bessel filters $(B, V, R, I)$. For each object, from 5 to 9 photometric points per band were obtained for the time interval between October 16 and December 16,2020 .

\footnotetext{
2 http://sln.oact.inaf.it/sln_old/dmdocuments/ ccd91rappint2-07.pdf

3 https://WWW . aavso.org/aavsonet

4 http://www.sdss3.org/dr8/algorithms/

sdssUBVRITransform. php\#Lupton2005
}

\subsection{Spectroscopic data reduction process}

Spectroscopic data reduction was carried out using the ESO Reflex workflow v2.8.5 (Freudling et al. 2013), specifically, the ESPRESSO v2.2.1 pipeline (Pepe et al. 2021), the UVES v6.1.3 pipeline (Ballester et al. 2000), and the X-shooter v3.5.0 pipeline (Modigliani et al. 2010). The pipelines carry out the standard steps of flat, bias, and dark correction, wavelength calibration, spectral rectification and extraction of the 1D spectra, and flux calibration using a standard star obtained in the same night. The UVES pipeline also allows extraction of individual spectra when two targets are present in the slit.

Additional steps are then performed as follows. The 1D extraction of the X-shooter spectra is carried out with IRAF $^{5}$ from the rectified flux-calibrated $2 \mathrm{D}$ spectrum in cases where the $\mathrm{S} / \mathrm{N}$ of the UVB arm is low, and for resolved binaries. For the latter cases, particular attention must be paid to the selection of the parameters for apertures definition and trace of the spectrum profile to maximize the signal of the extracted flux, avoiding to mix the spectra of two close stars.

Telluric correction is performed using the molecfit (Smette et al. 2015; Kausch et al. 2015) tool v3.0.3 for the VIS and NIR arms of X-shooter, and for the high-resolution ESPRESSO spectra. The latter is done by adapting the current molecfit workflow to the ESPRESSO data. The correction is always performed fitting the atmospheric model directly on the science spectra, since the $\mathrm{S} / \mathrm{N}$ on the continuum is always high enough to ensure a better correction with respect to using the telluric standard star observed in the same night to compute the model. For the UVES spectra, the telluric correction is performed using a standard telluric star spectrum obtained with the same instrument and same configuration. This is possible since only a few $\mathrm{O}_{2}$ telluric lines around the [O I] $\lambda 6300 \AA$ and $\mathrm{H}_{2} \mathrm{O}$ lines around $\mathrm{H} \alpha$ and $\mathrm{Na} \mathrm{I}_{2}$ are present; they can be easily identified and removed thanks to an IDL $^{6}$ procedure that allows us to align the telluric features by cross-correlation and rescaling of their intensity (see, e.g., Frasca et al. 2000)

Finally, the X-shooter spectra obtained with the narrow slits are scaled to the wide-slit ones to correct for slit losses. This procedure has already been tested in previous works, for example, Mendigutía et al. (2013), Alcalá et al. (2017), Manara et al. (2016a, 2017b), Rugel et al. (2018), and Kóspál et al. (2020), and leads to a typical absolute flux calibration accuracy of $\sim 10 \%$. The reduced, flux-calibrated, and telluric-corrected spectra are available on Zenodo ${ }^{7}$ in the ODYSSEUS data community ${ }^{8}$, and will also be made available also on the ESO Archive Phase 3 service.

\subsection{Data analysis}

The data are first analyzed with two tools, named here as "fitter" and ROTFIT, as described below. The analysis is aimed at

\footnotetext{
5 IRAF is distributed by the National Optical Astronomy Observatories, which are operated by the Association of Universities for Research in Astronomy, Inc., under the cooperative agreement with the National Science Foundation. NOAO stopped supporting IRAF, see https: //iraf-community.github.io/

6 IDL (Interactive Data Language) is a registered trademark of Harris Corporation.

7 For the Orion sample, X-shooter data: https://zenodo.org/ record/4477091\#. YBMOdpNKjlx; UVES data: https://zenodo. org/record/4478360\#. YBPQRJNKhTY; ESPRESSO data: https: // zenodo.org/record/4478376\#. YBPQWpNKhTY

8 https://sites.bu.edu/odysseus/
} 
deriving the stellar, accretion, and photospheric properties of the targets.

\subsubsection{Fitter of broadband spectra to obtain stellar and accretion properties}

The analysis of the X-shooter spectra to derive stellar and accretion properties is carried out with the method originally described in Manara et al. (2013a) and later applied to a number of X-shooter studies of young stars (e.g., Alcalá et al. 2014, 2017; Manara et al. 2016a, 2017a; Venuti et al. 2019). In short, the observed spectrum is dereddened and fit with the sum of a photospheric template spectrum and a hydrogen slab model with uniform density and temperature gas to reproduce the continuum excess emission due to accretion. The grid of models used to find the best fit comprises Class III photospheric templates with spectral types (SpT) from G- to late M-type taken from Manara et al. (2013b, 2017b), different slab models, and a series of extinction values $\left(A_{V}\right)$ all assuming the reddening law by Cardelli et al. (1989) and $R_{V}=3.1$. The integrated flux of the best fit slab models gives an estimate of the excess luminosity due to accretion $\left(L_{\mathrm{acc}}\right)$, and the best fit normalization of the Class III templates gives an estimate of the stellar luminosity $\left(L_{\star}\right)$. By converting the $\mathrm{SpT}$ to effective temperature $\left(T_{\text {eff }}\right)$ using the relation by Luhman et al. (2003), together with Kenyon \& Hartmann (1995), as described by Manara et al. (2013a), we are able to position the targets on the HR diagram. By comparing the position on the HR diagram with an interpolated set of evolutionary models by Baraffe et al. (2015) or, if $M_{\star}>1.4 M_{\odot}$, by Siess et al. (2000), it is possible to infer the stellar mass $\left(M_{\star}\right)$ of the targets. The choice of evolutionary models is done in line with previous works (e.g., Alcalá et al. 2017; Manara et al. 2017a). Finally, the mass accretion rate $\left(\dot{M}_{\text {acc }}\right)$ is obtained from the classic relation $\dot{M}_{\text {acc }}=1.25 \cdot L_{\text {acc }} R_{\star} /\left(G M_{\star}\right)$ (e.g., Hartmann et al. 1998).

As several emission lines are present in the X-shooter spectra, we measure their luminosity ( $\left.L_{\text {line }}\right)$ and convert them to $L_{\text {acc }}$ using the relations by Alcalá et al. (2017). Typically, the values of $L_{\text {acc }}$ obtained from the fitter described above and the mean value of $L_{\text {acc }}$ derived from the emission line fluxes are similar within the uncertainties (e.g., Herczeg \& Hillenbrand 2008; Alcalá et al. 2014, 2017). The presence of multiple accretion tracers along the wide wavelength coverage of the $\mathrm{X}$-shooter spectra provides an additional check on the $A_{V}$ estimates.

\subsubsection{Photospheric properties from ROTFIT}

The estimate of the photospheric properties $T_{\text {eff }}$ and $\log g$, radial (RV) and projected rotational velocity $(v \sin i)$, and veiling is performed on both the medium-resolution and the highresolution spectra using the ROTFIT code, already tested both on X-shooter data and on the higher resolution UVES spectra from the Gaia ESO Survey (Frasca et al. 2015, 2017).

ROTFIT uses a grid of template spectra to perform a $\chi^{2}$ minimization of the difference between the observed and template parameters in selected spectral regions. To construct the grid of templates, we collected different photospheric templates with spectral types similar to our targets. We built two grids of templates of high-resolution spectra of real slowly rotating stars with a low activity level: the first one includes spectra retrieved from the ELODIE archive $(R \simeq 42000$, Moultaka et al. 2004), which are the same used for the analysis of young stars within the Gaia-ESO survey by the OACT node (Frasca et al. 2015); the second is composed of spectra of KM-type stars retrieved from the HARPS archive $(R \simeq$ $115000)^{9}$. The main parameters of the HARPS templates are reported in Table C.1 along with references for the quoted stellar parameters.

To perform the $\chi^{2}$ minimization, when using the grid of ELODIE templates, the UVES and ESPRESSO spectra are convolved with a Gaussian kernel and resampled to match the resolution of ELODIE $(R=42000)$. For the analysis with the HARPS grid we have degraded the ESPRESSO spectra to $R=$ 115000 , while the reverse was done for the UVES ones, that is, the HARPS templates were brought to the UVES resolution $(R=70000)$ and resampled on the points of the target spectra. For the determination of photospheric parameters, $v \sin i$, and veiling from high-resolution spectra, we prefer real over synthetic spectra, because generally the former better reproduce the unknown photospheric spectrum. Some photospheric lines may be missing in the synthetic spectra, or the depths and widths of some of them may be poorly reproduced due to uncertain intensity values, Landé factors and broadening effects. However, as the non-active templates are mostly main-sequence stars, we need synthetic spectra for a safer determination of $\log g$. To this aim, BT-Settl synthetic spectra (Allard et al. 2012) are used to fit specific spectral regions containing gravity-sensitive features.

For the X-shooter spectra, which span a much wider wavelength range, we use a grid of BT-Settl synthetic spectra with a solar iron abundance (Allard et al. 2012). The radial velocity is calculated by means of the cross-correlation of template and target spectrum in specific spectral regions free from broad features and emission lines. The $v$ sin $i$ and veiling values are treated as free parameters in the fitting procedure and the best values are found by the $\chi^{2}$ minimum.

The photospheric lithium-poor templates with no sign of accretion and absent (or negligible) chromospheric emission in the cores of strong lines fitted by ROTFIT (including rotational broadening and veiling) can be subtracted to remove the photospheric lines. This has the great advantage of emphasizing emission features against underlying photospheric absorption lines, but it is also very important to clean the Li I $16707.8 \AA$ line from blended nearby lines, which, unlike Li I $\lambda 6707.8 \AA$, are present in the templates. Finally, this enables us to remove strong photospheric lines around emission lines tracing outflows and winds, such as the [OI] $\lambda 6300 \AA$ line (see Figs. 1, H.1, and H.2 for a few examples). The line profiles corrected for telluric absorption and for photospheric absorption lines will be provided to the community through Zenodo and the ESO Phase 3 Archive, and will serve as a great dataset to study the accretion and ejection mechanisms (e.g., Banzatti et al. 2019; McGinnis et al. 2018; Simon et al. 2016; Rigliaco et al. 2013).

\section{First PENELLOPE data: Young stars in Orion}

The first observations of the ULLYSES and PENELLOPE programs have focused on targets located in the Orion region. In particular, ten targets are part of the Orion OB1 association, and three of the $\sigma$-Orionis cluster. In the following, we briefly introduce the two regions, and report the properties from the literature, including the distances from the recent Gaia EDR3 release (Gaia Collaboration 2016, 2021), for the 13 targets analyzed here (see Table 1).

9 http://archive.eso.org/wdb/wdb/adp/phase3_main/form 


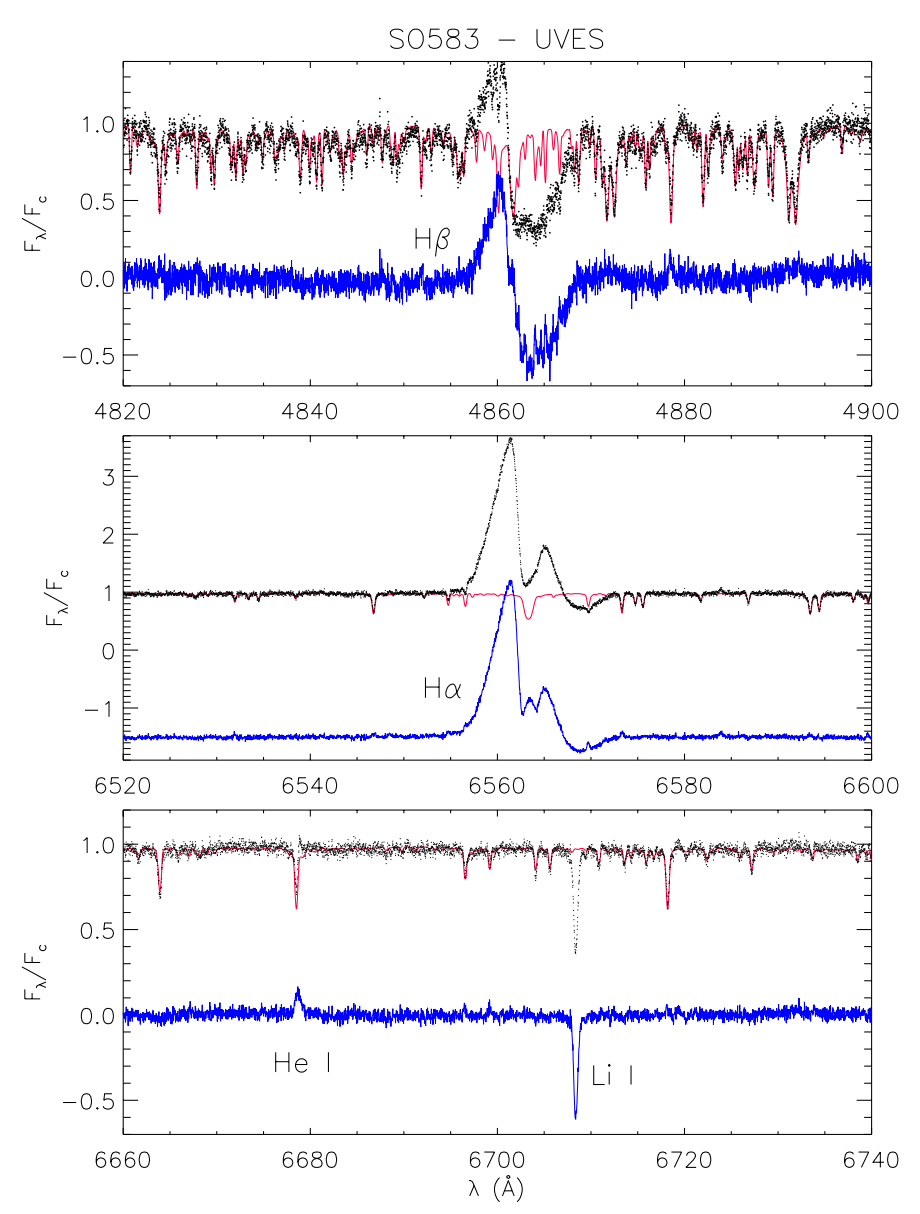

Fig. 1. Subtraction of the non-active, lithium-poor template (red line) from the spectrum of SO 583 (black dots), which reveals the complex structure of the $\mathrm{H} \beta$ and $\mathrm{H} \alpha$ lines (blue lines in the upper and central panel, respectively) and emphasizes the Li I $26707.8 \AA$ absorption line, removing the nearby blended lines (bottom panel). The He I $\lambda 6678.2 \AA$ emission line clearly emerges in the subtracted spectrum.

\subsection{Information on the Orion sample}

The Orion OB1 association is one of the closest and most populous $\mathrm{OB}$ associations, encompassing subassociations that range in age from $\sim 1$ Myr to $\sim 10$ Myr (Blaauw 1964). The low-mass counterparts to the OBA-type stars characterizing the association were first identified in the CIDA Variability Survey (CVSO, Briceño et al. 2001, 2005, 2019), carried out using the Quest camera in the $J$. Stock telescope in Venezuela and recently confirmed spectroscopically (cf. Briceño et al. 2019). Circumstellar disks were discovered and characterized with Spitzer (Hernández et al. 2007a) and Herschel (Maucó et al. 2018). The X-ray luminosities and accretion properties of some of the CVSO objects, including HST observations, have been discussed in Ingleby et al. $(2009,2011,2014)$ and Thanathibodee et al. (2018, 2019).

The HST/ULLYSES team selected ten targets in the Orion OB1a and OB1b subassociations. Their spectral types are between K6 and M3, and most of them have low interstellar extinction $\left(A_{V}<0.3 \mathrm{mag}\right.$, Briceño et al. 2019). Eight targets with signatures of accretion are located in Orion OB1b, which has an age of $\sim 5 \mathrm{Myr}$, while the two non-accreting targets, CVSO 17 and CVSO 36, are located in the 25 Ori group within the Orion OB1a subassociation, with ages of $\sim 10 \mathrm{Myr}$ (Briceño et al. 2019).
In addition to distributed populations, the Orion OB1 association includes several stellar clusters such as the $\sigma$ Ori cluster. Low-mass members of $\sigma$ Ori were first reported by Walter et al. (1997), who found over 80 X-ray sources and spectroscopically identified more than 100 low-mass, pre-main-sequence (PMS) members lying within $1^{\circ}$ from the star $\sigma$ Ori, which is in fact a massive quintuplet system of $\mathrm{O}$ and early $\mathrm{B}$ stars (Caballero 2014). With an estimated age of $\sim 3-5 \mathrm{Myr}$ (Caballero 2018), the $\sigma$ Ori cluster is of interest because of its intermediate age and for being an excellent laboratory to study protoplanetary disks in dense environments containing OB stars, where far-ultraviolet radiation fields from massive stars can externally illuminate the disks, producing photoevaporating winds.

Extensive studies of membership to the $\sigma$ Orionis cluster were carried out by Hernández et al. (2014) and Caballero et al. (2019). Its protoplanetary disks have been followed with Spitzer (Hernández et al. 2007b), Herschel PACS (Maucó et al. 2016), and ALMA (Ansdell et al. 2017). Estimates of accretion rates for members of this cluster were obtained with $U$-band photometry by Rigliaco et al. (2011), and, for a small sub-sample, with X-shooter spectroscopy (Rigliaco et al. 2012). The HST/ULLYSES team selected three targets in $\sigma$ Ori, with low extinction and clear signs of accretion (Maucó et al. 2016). Information on the targets taken from the literature is presented in Appendix A and summarized in Tables A.1 and A.2.

\subsubsection{Distances from Gaia parallaxes}

The Gaia EDR3 astrometric solutions for the ten targets are generally quite good, showing, for example, low renormalized unit weight errors (RUWEs). Only the astrometric solution for CVSO 17 has RUWE $=1.8$ and thus slightly exceeds the value of $\operatorname{RUWE}=1.4$, which is considered an appropriate nominal limit for Gaia EDR3. Stars with RUWE > 1.4 could have an ill-behaved astrometric solution (Gaia Collaboration 2021). For these, we compare the distance obtained by inverting the parallax (arithmetic distances), after correcting for the zero points estimated from the analytical functions of Lindegren et al. (2021), with the geometric Bayesian distance estimated by BailerJones et al. (2021). The differences between the arithmetic and Bayesian distance are smaller than $1 \mathrm{pc}$. Stars with reliable astrometric solutions have uncertainties in parallaxes around $1 \%$. Thus, we assumed the arithmetic distances for these targets (Table 1). Bailer-Jones (2015) suggests that we cannot apply the inverse relation between parallaxes and distances to stars with fractional parallax errors larger than $20 \%$, where the estimation of distances becomes an inference problem in which the use of prior assumptions is necessary.

Different stellar groups in the Orion star-forming complex can be detected as kinematically distinct populations with characteristic proper motions, radial velocities, and distances (Kounkel et al. 2018). In our case, the distances obtained for the 10 targets with reliable astrometric solutions, together with the radial velocities from Kounkel et al. (2018) and measured here (Sect. 4.2), are consistent with the expected values for the Orion OB $1 \mathrm{a} / \mathrm{OB} 1 \mathrm{~b}$ sub association, and the $\sigma$-Ori cluster, respectively. Even CVSO 17, which has a value of RUWE $=1.8$, has a distance consistent with the upper boundary of the distance distribution of the 25 Ori cluster (Briceño et al. 2019), different from the distance obtained for the other star in the cluster (CVSO 36; $345 \mathrm{pc}$ ). Regardless of the relative large fractional parallax error of CVSO 17 (22\%), the arithmetic distance reported in Table 1 agrees with the geometric Bayesian distance, $415.3_{-10.1}^{+9.7} \mathrm{pc}$, estimated by Bailer-Jones et al. (2021). The assumed distance for CVSO 17 has the caveat that the value of RUWE is just above the usual limit. For CVSO 109 and CVSO 165, for which no reliable 
Table 1. Stellar and accretion properties from this work for the targets in the Orion OB1 Association and $\sigma$-Orionis cluster.

\begin{tabular}{lllllccccccc}
\hline \hline Target & Other name & $\begin{array}{c}\mathrm{RA}_{2000} \\
\text { hh:mm:ss.s }\end{array}$ & $\begin{array}{c}\text { Dec 2000 } \\
\text { dd:mm:ss.s }\end{array}$ & $\begin{array}{c}\text { Parallax } \\
{[\mathrm{mas}]}\end{array}$ & $\begin{array}{c}\text { Dist. } \\
{[\mathrm{pc}]}\end{array}$ & $\mathrm{SpT}$ & $\begin{array}{c}A_{V} \\
{[\mathrm{mag}]}\end{array}$ & $\begin{array}{c}L_{\star} \\
{\left[L_{\odot}\right]}\end{array}$ & $\begin{array}{c}\log L_{\mathrm{acc}} \\
{\left[L_{\odot}\right]}\end{array}$ & $\begin{array}{c}M_{\star} \\
{\left[M_{\odot}\right]}\end{array}$ & $\begin{array}{c}\log \left(\dot{M}_{\mathrm{acc}}\right) \\
{\left[M_{\odot} \mathrm{yr}^{-1}\right]}\end{array}$ \\
\hline CVSO 17 & $\ldots$ & $05: 23: 04.72$ & $01: 37: 15.3$ & $2.4144 \pm 0.0531$ & $414.2_{-8.9}^{+9.3}$ & $\mathrm{M} 2$ & 0.0 & 0.30 & $-4.62^{(*)}$ & 0.37 & $-11.43^{(*)}$ \\
CVSO 36 & $\ldots$ & $05: 25: 50.37$ & $01: 49: 37.3$ & $2.9327 \pm 0.0265$ & $335.5 \pm 3.0$ & $\mathrm{M} 2$ & 0.1 & 0.22 & $-3.42^{(*)}$ & 0.39 & $-10.31^{(*)}$ \\
CVSO 58 & $\ldots$ & $05: 29: 23.26$ & $-01: 25: 15.5$ & $2.8229 \pm 0.0231$ & $349.0 \pm 2.8$ & $\mathrm{~K} 7$ & 0.8 & 0.32 & -1.12 & 0.81 & -8.37 \\
CVSO 90 & $\ldots$ & $05: 31: 20.63$ & $-00: 49: 19.8$ & $2.9107 \pm 0.033$ & $338.7_{-3.7}^{3.8}$ & $\mathrm{M} 0.5$ & 0.1 & 0.13 & -1.34 & 0.62 & -8.61 \\
CVSO 104 & Haro 5-64 & $05: 32: 06.49$ & $-01: 11: 00.8$ & $2.7296 \pm 0.0298$ & $360.7_{-3.8}^{+3.9}$ & $\mathrm{M} 2$ & 0.2 & 0.37 & -1.73 & 0.37 & -8.49 \\
CVSO 107 & $\ldots$ & $05: 32: 25.79$ & $-00: 36: 53.4$ & $2.9843 \pm 0.0226$ & $330.4 \pm 2.5$ & $\mathrm{M} 0.5$ & 0.3 & 0.32 & -1.30 & 0.53 & -7.30 \\
CVSO 109 & V462 Ori & $05: 32: 32.66$ & $-01: 13: 46.1$ & $\ldots$ & 400 & $\mathrm{M} 0.5$ & 0.1 & 0.92 & -0.77 & 0.46 & -7.49 \\
CVSO 146 & V499 Ori & $05: 35: 46.01$ & $-00: 57: 52.2$ & $2.9701 \pm 0.0152$ & $332.0 \pm 1.7$ & $\mathrm{~K} 6$ & 0.6 & 0.80 & -1.46 & 0.86 & -8.57 \\
CVSO 165 & $\ldots$ & $05: 39: 02.57$ & $-01: 20: 32.3$ & $\ldots$ & 400 & $\mathrm{~K} 6$ & 0.2 & 0.98 & -2.05 & 0.84 & -9.10 \\
CVSO 176 & V609 Ori & $05: 40: 24.15$ & $-00: 31: 21.3$ & $3.2593 \pm 0.0314$ & $302.4_{-2.8}^{+2.9}$ & $\mathrm{M} 3.5$ & 1.0 & 0.34 & -1.27 & 0.25 & -7.84 \\
\hline SO 518 & V505 Ori & $05: 38: 27.26$ & $-02: 45: 09.7$ & $2.5064 \pm 0.025$ & $392.3_{-3.8}^{+3.9}$ & $\mathrm{~K} 7$ & 1.0 & 0.24 & -1.22 & 0.81 & -8.53 \\
SO 583 & TX Ori & $05: 38: 33.69$ & $-02: 44: 14.1$ & $\ldots$ & 385 & $\mathrm{~K} 5$ & 0.4 & 3.61 & -0.30 & 1.09 & -7.21 \\
SO 1153 & V510 Ori & $05: 39: 39.83$ & $-02: 31: 21.9$ & $2.5212 \pm 0.0268$ & $390.3_{-4.0}^{+4.1}$ & $\mathrm{~K} 7$ & 0.1 & 0.17 & -0.88 & 0.76 & -8.24 \\
\hline
\end{tabular}

Notes. Values obtained fitting the X-shooter spectra. ${ }^{(*)}$ These values should be considered as upper limits since the targets are not-accreting.

astrometric solution is available, we assume the mean distance of the Orion OB1b sub-association, 400 pc (Briceño et al. 2019), and for SO 583, we assume the mean distance to the $\sigma$-Orionis cluster, 385 pc (Maucó et al. 2016).

\subsubsection{Multiplicity of the targets}

Multiplicity for several of the targets in the Orion OB1 association was recently studied by Tokovinin et al. (2020), who reported that the following targets are physical binaries: CVSO 17 (separation $\sim 8.1^{\prime \prime}$, same companion present also in Gaia EDR3), CVSO 36 (sep 3.3"), CVSO 109 (sep 0.7").

In two other cases, a visual companion is a background object: CVSO 104 (sep 2.35", same nearby object present also in Gaia EDR3, with parallax $=1.49$ mas, thus background), CVSO 165 (sep 5.4", same nearby object present also in Gaia EDR3, also background). For CVSO 165, the observations of the HST/ULLYSES program reveal that the primary component is actually a $\sim 0.3^{\prime \prime}$ binary (Proffitt et al. 2021), which we do not resolve in our VLT observations. The same observations also confirm the binarity of CVSO 109 (Proffitt et al. 2021). No binary components were found for CVSO 58, CVSO 90, CVSO 107, and CVSO 146, and no observations for CVSO 176 were carried out by Tokovinin et al. (2020).

In our VLT observations, we cannot resolve the close $\left(<1^{\prime \prime}\right)$ binary systems CVSO 109 and CVSO 165, whereas the other binaries are resolved. Moreover, we observe from our multiepoch high-resolution spectra that the primary component of CVSO 104 is a spectroscopic binary.

\subsection{Observation and data reduction for the first PENELLOPE dataset}

The first observing run of our PENELLOPE Large Program happened soon after VLT was brought back into operations after the shutdown due to the COVID-19 pandemic. During the first two weeks of observations, only X-shooter and UVES, among the instruments selected for our program, were operational. We thus obtained observations of our bright targets with UVES instead of ESPRESSO in this period. The first observation with UVES was on the target CVSO 104 and happened on November 25, 2020, while the first X-shooter observation was performed on November 27, 2020. It was only on December
8, 2020 that ESPRESSO came back into operation and could be used to observe SO 1153. The last spectra of this run were obtained on December 15, 2020. A technical issue on UT3 made X-shooter unavailable for three nights, and a technical problem on ESPRESSO made it unavailable for a few days after December 15, 2020. This has caused a larger than desired time separation between the HST and X-shooter observations for CVSO 176, and resulted in having only one epoch of highresolution spectra for CVSO 90.

Figure B.1 shows the time of the observations with VLT and HST. In summary, for our 13 targets, we typically obtained two high-resolution spectra in advance of the HST observations, and one during or after the HST observations. It is only in the case of SO 1153 that the three spectra were obtained after the HST observations and that is because of the initial unavailability of ESPRESSO. The three epochs with high-resolution spectroscopy were all obtained in three consecutive nights. In most cases the X-shooter observations were obtained on the night of the HST/STIS observations or the night before, with the exception of CVSO 176, observed with X-shooter two nights after the HST/STIS observations, and of SO 518 and SO 583, both observed one night after HST/STIS due to the unavailability of X-shooter on the preceding nights. With those few exceptions, the plan of our observations (as described in Sect. 2.2.3) was successfully implemented. The observations log and additional information is presented in Tables B.1-B.3. Observations were carried out under excellent weather conditions and typically resulted in an image quality of $\sim 1^{\prime \prime}$.

Reduction of the spectroscopic data is carried out as described in Sect. 2.4. Here we briefly mention the peculiarities relative to this dataset. The $1 \mathrm{D}$ spectra have been obtained using the standard extraction provided by the pipelines, with the only exception of the UVB arms of the X-shooter spectra of SO 518 and SO 583, which were extracted with IRAF from the 2D flux calibrated spectra produced by the pipeline. This was needed since the image quality parameter at the time of the observations $\left(\sim 1.1^{\prime \prime}-1.2^{\prime \prime}\right)$ was much larger than the slit width $\left(0.5^{\prime \prime}\right)$ used only for these targets in this arm, and the manual extraction with IRAF improved the $\mathrm{S} / \mathrm{N}$ of the spectra. Also, the X-shooter spectra of CVSO 104, and of its wide visual companion observed in the slit, were extracted manually with IRAF. The spectrum of the companion (located to the east of the target) is not analyzed here 

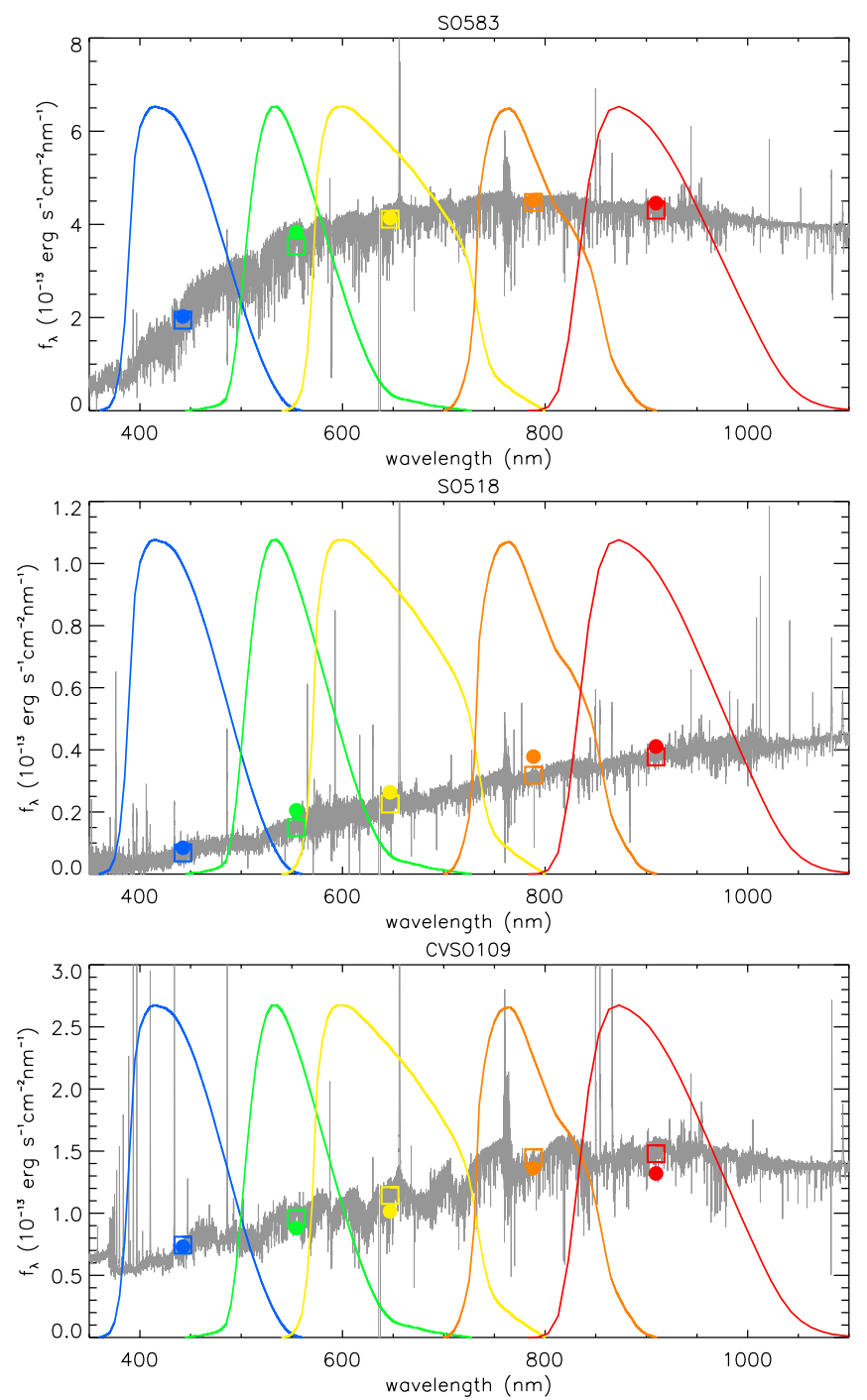

Fig. 2. X-shooter flux-calibrated spectrum of three of our targets (grey), as labelled, with quasi-simultaneous photometry for the same target obtained from OACT in the $B V R_{\mathrm{C}} I_{\mathrm{C}} z^{\prime}$ bands (dots). The filter bandpasses and the synthetic photometry (open squares) obtained integrating the spectrum over these bandpasses are overlaid.

since this is a background star. The UVES spectra of this same target and its visual companion were instead extracted with the pipeline, adapting the extraction window to the position of the two traces in the $2 \mathrm{D}$ spectra.

The rescaling of the narrow slit X-shooter spectra to those obtained with a wide slit are done using a single scaling factor in the UVB and a linearly wavelength dependent scaling factor in the VIS and NIR arms. An example of the achieved flux calibration of the X-shooter spectra is shown in Fig. 2. The overall agreement between the photometry obtained within less than between two and four hours from the X-shooter spectrum is typically within $10 \%$; in the worst case, which is our assumed (and possibly overestimated) uncertainty on the absolute flux calibration of the X-shooter spectra. In the case of SO 518, the photometric fluxes are systematically higher, at all wavelengths, while for CVSO 109 they are all lower than those measured on the X-shooter spectrum. This can be understood by looking at the light curves (Fig. D.1). Indeed, OACT photometric data are taken about two hours before the X-shooter spectrum for SO 518, whose brightness was dimming, and four hours before the X-shooter spectrum for CVSO 109, which was brightening.
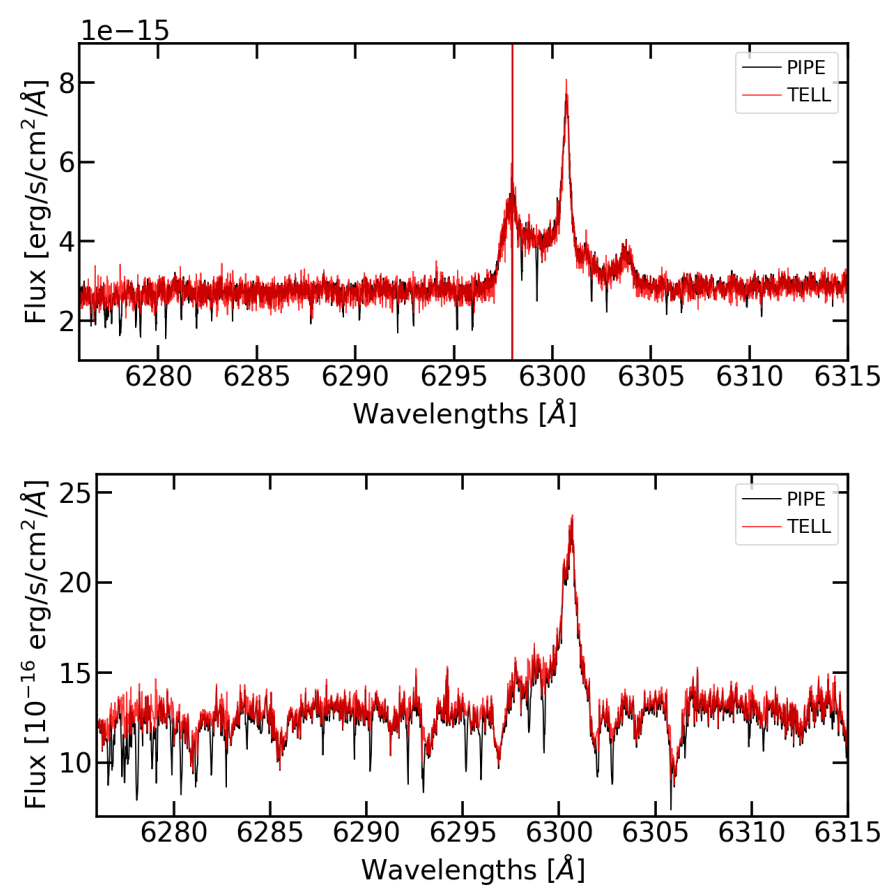

Fig. 3. Example of telluric correction in the region of the $[\mathrm{OI}] 6300 \AA$ line for the ESPRESSO spectrum of CVSO 90 performed with molecfit (top) and for the UVES spectrum of CVSO 58 carried out with a standard telluric (bottom). The black spectrum is obtained from the pipeline reduction, and the red one shows the telluric corrected spectrum.

Telluric correction leads to good results in most of the VIS spectra and for the ESPRESSO spectra. Only the regions with very deep $\mathrm{O}_{2}$ telluric absorption lines at around $\sim 760 \mathrm{~nm}$, and sometimes the one around $\sim 690 \mathrm{~nm}$, are poorly corrected. In particular, these absorption lines are saturated in the ESPRESSO data, and are therefore not properly corrected. In the X-shooter spectra, the correction of the $\mathrm{H}_{2} \mathrm{O}$ bands at around $\sim 950 \mathrm{~nm}$ and $\sim 1130 \mathrm{~nm}$ leaves in some cases strong residuals. The correction from telluric lines around the [OI]6300 $\AA$ line is always good, as shown in Fig. 3. Similarly, the telluric correction of the UVES spectra using a telluric standard star observed with the same instrument configuration allows us to properly remove the telluric absorption features around the $[\mathrm{OI}] 6300 \AA$ line, as shown in Fig. 3.

\section{Analysis of the Orion data}

Here, we present the analysis of the X-shooter, UVES, and ESPRESSO data obtained in the first run of PENELLOPE, focusing on the stellar, photospheric, and accretion properties of the whole sample. Detailed analysis of the individual spectra, focusing on the study of the ejections from the emission line profiles, on the kinematic properties of the accretion process, and of the binarity of the targets and more, are deferred to forthcoming works.

\subsection{Stellar and accretion parameters from the $X$-shooter spectra}

Figure 4 shows the best fit of the X-shooter spectrum of the CVSO 176 source obtained with the method described in Sect. 2.5.1, while best fits for the entire sample are shown in Figs. E.1-E.4. In general, the fits reproduce well the Balmer 

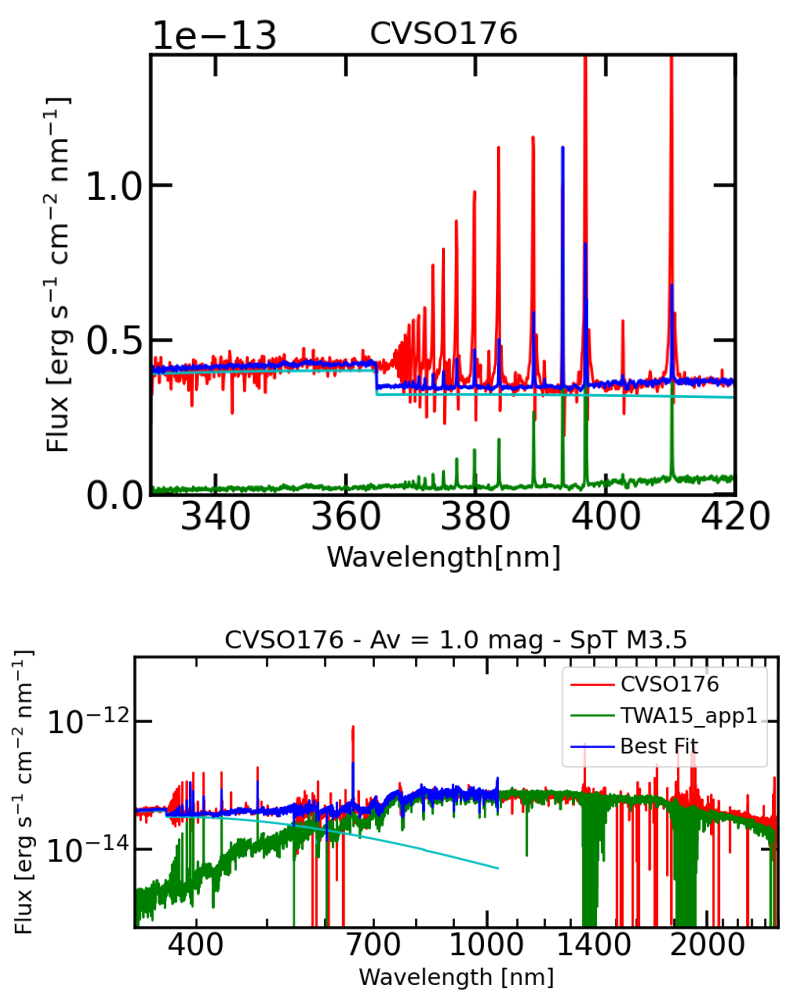

Fig. 4. Example of a best fit for the X-shooter spectrum of CVSO 176 obtained with the fitter described in Sect. 2.5.1. The spectrum of the target is shown with a red line, the best fit photospheric template with a green line, the slab model with a cyan line, and the best-fit sum of the template and slab model with a blue line. The best fit is only shown up to $1000 \mathrm{~nm}$, since the contribution from the disk emission can be significant at longer wavelengths.

jump and the continuum of the targets up to $\sim 1000 \mathrm{~nm}$. In the NIR, a region of the spectrum that is not considered when running the fitter, the best fit is in most cases fainter than the data, which is expected since the flux contribution from the disk emission at NIR wavelengths is not considered in the modeling. The values of spectral type, $A_{V}, L_{\star}, M_{\star}, L_{\text {acc }}$, and $\dot{M}_{\text {acc }}$ derived from the fit are listed in Table 1. Typical uncertainties on those parameters are $0.1 \mathrm{mag}, 0.2 \mathrm{dex}, 0.1 \mathrm{dex}, 0.25 \mathrm{dex}$, and $0.45 \mathrm{dex}$, as described by Alcalá et al. $(2014,2017)$ and Manara et al. (2013b).

Our analysis of the X-shooter spectra of CVSO 17 and CVSO 36 shows that these are non-accreting stars. A similar conclusion can be reached in considering the HST spectra (Pittman, in prep.). We can consider the estimated values of $L_{\text {acc }}$ as upper limits to the accretion rates, which thus fall well below the typically assumed detection limits for accretion rates due to chromospheric activity discussed by Manara et al. (2013b, 2017b).

As expected, the best fits are generally obtained with low values of $A_{V} \lesssim 0.5 \mathrm{mag}$. It is only in the case of CVSO 176 and SO 518 that we obtain $A_{V}=1 \mathrm{mag}$, along with $A_{V}=0.8 \mathrm{mag}$ for CVSO 58. These values are in line with previous literature estimates (see Table A.1). They are also consistent with the values that minimize the spread of $L_{\text {acc }}$ obtained converting the line luminosity of several lines in the X-shooter spectrum using the relations by Alcalá et al. (2017).

The target CVSO 104 was reported to be a K7 star in the literature, which may be due to its binarity. Looking at our spectra, the fit improves assuming a later spectral type. A more detailed

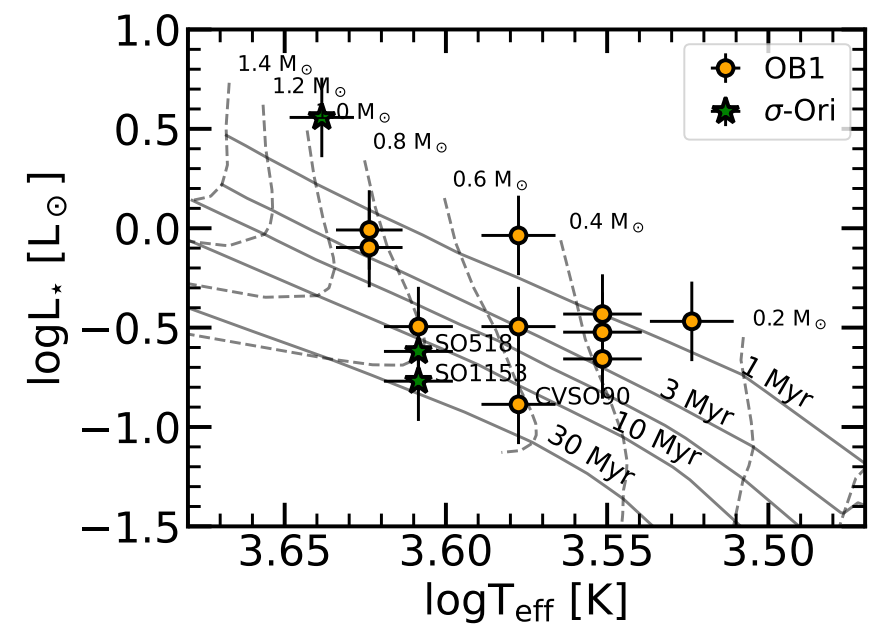

Fig. 5. Hertzprung-Russel diagram (HRD) of the Orion targets, with evolutionary tracks from Baraffe et al. (2015). The isochrones shown here are for ages of $1,3,5,10$, and $30 \mathrm{Myr}$.

analysis of this target that carefully takes its binary status into account will be performed in a forthcoming paper.

The Hertzprung-Russel diagram (HRD) of the data analyzed here is shown in Fig. 5. Several targets lie between the 1 and $10 \mathrm{Myr}$ isochrones of Baraffe et al. (2015), with only SO 518, SO 1153, and CVSO 90 lying between the $10 \mathrm{Myr}$ and 30 Myr isochrones. Cody \& Hillenbrand (2010) reported SO 518 and SO 1153 to be aperiodic type, with typical $\Delta I$ of 0.8 and $0.5 \mathrm{mag}$, respectively. At the time of our observations, SO 518 and CVSO 90 are affected by some significant $(\Delta V>0.5 \mathrm{mag})$ photometric variability, in particular by dimming events. We can see from Figs. 2 and D.1 that SO 518 was observed during a dimming event of more than $1 \mathrm{mag}$ in the $V$-band. The value of $L_{\star}$ measured on the spectrum is therefore lower than the one we would measure at its peak brightness, as the extinction we calculate here does not account for grey-extinction effects. We note that such a dimming event would affect also the measured accretion luminosity, as described in the extreme case of edge-on disks in Alcalá et al. (2014). Another aspect to consider is that a different relation between $T_{\text {eff }}$ and SpT (e.g., Pecaut \& Mamajek 2013; Herczeg \& Hillenbrand 2014) would affect the position of the target on the HRD, moving the star to a colder temperature, and thus an apparently younger isochronal age. This is also the case for SO 1153.

\subsection{Photospheric parameters and veiling from high-resolution spectra}

Both the high-resolution UVES and ESPRESSO spectra and the $\mathrm{X}$-shooter spectra are analyzed with the ROTFIT tool, described in Sect. 2.5.2 and in Frasca et al. (2015, 2017). The results obtained on the UVES spectra using the HARPS templates are reported in Table 2, those on the ESPRESSO data in Table 3, and those on the X-shooter spectra in Table 4. Strongly veiled objects or those with too many emission lines in their spectra, in particular CVSO 90 and SO 1153, are challenging for the analysis with ROTFIT. The results for the former are not reported, while for the latter, they are added here but they are more uncertain than for the other targets. For all the accreting objects, the values of $\log g$ are also quite uncertain and will be re-examined in a future work. 
Table 2. Photospheric properties from the UVES spectra fit with ROTFIT for the targets in the Orion OB1 Association and $\sigma$-Orionis cluster.

\begin{tabular}{|c|c|c|c|c|c|c|c|c|c|c|c|}
\hline Name & och & $\begin{array}{c}\text { HJD } \\
2459100+\end{array}$ & $\begin{array}{l}T_{\text {eff }} \\
{[\mathrm{K}]}\end{array}$ & $\log g$ & $\mathrm{SpT}$ & $\begin{array}{r}v \sin i \\
{\left[\mathrm{~km} \mathrm{~s}^{-1}\right]} \\
\end{array}$ & $\begin{array}{c}\mathrm{RV} \\
{\left[\mathrm{km} \mathrm{s}^{-1}\right]}\end{array}$ & r500 & r550 & r600 & r650 \\
\hline & ep1 & & $3721 \pm 72$ & & & 8 & & & & & \\
\hline & & & & & & & & & & & \\
\hline 7 & & & & & & & & & & & \\
\hline$\overline{\mathrm{VS}}$ & & & & & & & & & & & \\
\hline & & & & & & & & & & & \\
\hline CVSO 36 & & & & & V & & & & & & \\
\hline $\mathrm{CVS}$ & & & & & & & & & & & \\
\hline & & & & & V & & & & & & \\
\hline 8 & & & & 4.6 & $7 \mathrm{~V}$ & & & & & & \\
\hline CVS & & & & & & & & & & & \\
\hline & & & & & & & & & & & \\
\hline 7 & ep3 & & & & V & & & & & & \\
\hline & & & & & & & 0.40 & & & & $=0.08$ \\
\hline & & & & & & & & & & & \\
\hline & ер3 & & & 4.6 & MOV & & 38 & & & 0.6 & 0.14 \\
\hline & & & & & & & & & & $0.90 \pm 0.55$ & $0.93 \pm 0.60$ \\
\hline & & & & & & & & & & & \\
\hline & 4 & & & & M3V & & & & & & 0.6 \\
\hline & & & & & $\mathrm{K} 7$ & & & & & & 1.2 \\
\hline & & & & & $\mathrm{K}$ & & & & & & \\
\hline & ep3 & & & & K6 & & & & & & 0.8 \\
\hline & & & & & $\mathrm{K} 3$ & & & & & & \\
\hline & & & & & $\mathrm{K} 3$ & & & & & & \\
\hline SO 583 & ep3 & 63973 & $4725 \pm 117$ & $4.59 \pm 0.10$ & K4V & $10.8 \pm 1.5$ & $29.61 \pm 0.34$ & $0.49 \pm 0.21$ & $0.95 \pm 0.07$ & $0.84 \pm 0.05$ & $0.80 \pm 0.07$ \\
\hline
\end{tabular}

Notes. Values obtained fitting the UVES spectra with the HARPS templates. The measured veiling at different wavelengths is reported in the column labelled ' $r$ ' followed by the wavelength in $\mathrm{nm}$.

Table 3. Photospheric properties from the ESPRESSO spectra fit with ROTFIT for the targets in the Orion OB1 Association and $\sigma$-Orionis cluster.

\begin{tabular}{|c|c|c|c|c|c|c|c|c|c|c|c|}
\hline Name & Epoch & $\begin{array}{c}\text { HJD } \\
2459100+\end{array}$ & $\begin{array}{l}T_{\text {eff }} \\
{[\mathrm{K}]}\end{array}$ & $\log g$ & SpT & $\begin{array}{r}v \sin i \\
{\left[\mathrm{~km} \mathrm{~s}^{-1}\right]}\end{array}$ & $\begin{array}{c}\mathrm{RV} \\
{\left[\mathrm{km} \mathrm{s}^{-1}\right]}\end{array}$ & $\mathrm{r} 500$ & $\mathrm{r} 550$ & r600 & r650 \\
\hline CVSO 146 & ep1 & 92.62465 & $4303 \pm 97$ & $4.66 \pm 0.11$ & K6V & $4.4 \pm 0.8$ & $20.70 \pm 0.29$ & $0.29 \pm 0.11$ & $0.38 \pm 0.07$ & $0.25 \pm 0.11$ & $0.28 \pm 0.04$ \\
\hline CVSO 146 & ер2 & 93.60337 & $4372 \pm 101$ & $4.63 \pm 0.10$ & K6V & $5.0 \pm 0.8$ & $20.40 \pm 0.30$ & $0.40 \pm 0.10$ & $0.44 \pm 0.10$ & $0.31 \pm 0.10$ & $0.34 \pm 0.09$ \\
\hline CVSO 146 & ep3 & 94.59294 & $4272 \pm 113$ & $4.70 \pm 0.10$ & K6V & $6.4 \pm 0.9$ & $19.82 \pm 0.31$ & $0.56 \pm 0.08$ & $0.60 \pm 0.02$ & $0.53 \pm 0.10$ & $0.42 \pm 0.08$ \\
\hline CVSO 165 & ep1 & 96.57902 & $4591 \pm 167$ & $4.60 \pm 0.10$ & K4V & $15.5 \pm 0.9$ & $27.94 \pm 0.59$ & $0.10 \pm 0.07$ & $0.28 \pm 0.04$ & $0.24 \pm 0.12$ & $0.25 \pm 0.05$ \\
\hline CVSO 165 & ep2 & 97.60896 & $4591 \pm 169$ & $4.60 \pm 0.10$ & $\mathrm{~K} 4 \mathrm{~V}$ & $15.4 \pm 0.9$ & $28.00 \pm 0.38$ & $0.13 \pm 0.05$ & $0.32 \pm 0.04$ & $0.28 \pm 0.07$ & $0.32 \pm 0.04$ \\
\hline CVSO 165 & ep3 & 98.61706 & $4585 \pm 167$ & $4.60 \pm 0.10$ & K4V & $15.2 \pm 0.8$ & $27.77 \pm 0.39$ & $0.21 \pm 0.05$ & $0.36 \pm 0.05$ & $0.29 \pm 0.11$ & $0.36 \pm 0.05$ \\
\hline SO 1153 & ep1 & 91.83202 & $4152 \pm 158$ & $4.67 \pm 0.10$ & K7V & $9.9 \pm 1.6$ & $34.43 \pm 0.57$ & $5.52 \pm 0.54$ & $5.54 \pm 0.85$ & $4.08 \pm 0.66$ & $4.81 \pm 0.62$ \\
\hline SO 1153 & ep2 & 92.59172 & $4119 \pm 181$ & $4.68 \pm 0.11$ & K7V & $9.1 \pm 1.2$ & $36.82 \pm 0.48$ & $6.28 \pm 0.71$ & $6.13 \pm 0.78$ & $5.08 \pm 0.71$ & $5.23 \pm 0.56$ \\
\hline SO 1153 & ep3 & 93.63656 & $4065 \pm 146$ & $4.69 \pm 0.11$ & K7V & $11.6 \pm 1.9$ & $34.66 \pm 0.57$ & $6.52 \pm 0.44$ & $6.83 \pm 0.85$ & $5.22 \pm 0.65$ & $5.71 \pm 0.80$ \\
\hline
\end{tabular}

Notes. Values obtained fitting the ESPRESSO spectra with the HARPS templates. The measured veiling at different wavelengths is reported in the column labelled ' $r$ ' followed by the wavelength in $\mathrm{nm}$.

The values of $T_{\text {eff }}$ and the spectral types obtained with ROTFIT are in good agreement with the ones derived by fitting the X-shooter spectrum with the method by Manara et al. (2013a). The comparison is shown in Figs. 6 and 7 for the UVES spectra. The spectral types obtained with the two methods are in good agreement and the differences in $T_{\text {eff }}$ are due to a different spectral type- $T_{\text {eff }}$ scale used for the HARPS templates with respect to the one used for the X-shooter fit. Similarly, the results obtained with ROTFIT on the X-shooter spectra are in good agreement with the values obtained on the same $\mathrm{X}$-shooter spectra with the fitter discussed in Sect. 2.5.1. This is shown in Fig. 8, and is very much in line with previous results (e.g., Manara et al. 2020; Frasca et al. 2017).

A comparison of the veiling values measured with ROTFIT with the equivalent width (EW) of the $\mathrm{H} \alpha$ lines shows a general correlated increase of the veiling with the strength of the
$\mathrm{H} \alpha$ emission line. This is shown in Fig. 9. The Spearman's rank correlation coefficient for the veiling at $650 \mathrm{~nm}, \rho=0.83$ with a significance $\sigma=2.6 \times 10^{-9}$ (Press et al. 1992) supports the high degree of correlation.

On the other hand, the comparison of the veiling values measured with ROTFIT on the UVES or ESPRESSO spectra with $\dot{M}_{\text {acc }}$ derived from X-shooter data, which are not exactly simultaneous, shows in general a larger value of $\dot{M}_{\text {acc }}$ at higher values of veiling, but with no clear correlation $(\rho=0.62, \sigma=$ $1.09 \times 10^{-4}$ ), possibly due to the narrow range of (high) $\dot{M}_{\text {acc }}$ covered by the observations. This is shown in Fig. 10. A similar degree of correlation ( $\rho=0.45, \sigma=0.27$ ) is found when comparing the veiling measurements from the X-shooter spectra to $\dot{M}_{\text {acc }}$ from the same spectra, as shown in Fig. 11. A further investigation of these correlations should be carried out with 
C. F. Manara et al.: PENELLOPE. I. Survey presentation and Orion

Table 4. Photospheric properties from the X-shooter spectra fit with ROTFIT for the targets in the Orion OB1 Association and $\sigma$-Orionis cluster.

\begin{tabular}{lccclcccc}
\hline \hline Name & $\begin{array}{c}\text { HJD } \\
2459100+\end{array}$ & $\begin{array}{c}T_{\mathrm{eff}} \\
{[\mathrm{K}]}\end{array}$ & $\log g$ & $\begin{array}{l}v \sin i \\
{\left[\mathrm{~km} \mathrm{~s}^{-1}\right]}\end{array}$ & $\begin{array}{c}\mathrm{RV} \\
{\left[\mathrm{km} \mathrm{s}^{-1}\right]}\end{array}$ & $\mathrm{r620}$ & $\mathrm{r} 710$ & $\mathrm{r} 970$ \\
\hline CVSO 17 & 88.62128 & $3588 \pm 22$ & $4.78 \pm 0.24$ & $<6.0$ & $27.4 \pm 1.9$ & 0.4 & 0.2 & 0.1 \\
CVSO 36 & 86.62703 & $3515 \pm 24$ & $4.58 \pm 0.14$ & $<6.0$ & $18.6 \pm 1.7$ & 0.4 & 0.4 & 0.1 \\
CVSO 58 & 85.76194 & $3886 \pm 45$ & $4.81 \pm 0.21$ & $19.3 \pm 1.0$ & $23.2 \pm 2.2$ & 1.0 & 0.2 & 0.5 \\
CVSO 90 & 98.56264 & $3489 \pm 32$ & $4.26 \pm 0.30$ & $13.0 \pm 16.0$ & $23.8 \pm 2.7$ & $\ldots$ & 1.8 & 1.4 \\
CVSO 107 & 87.58635 & $3766 \pm 37$ & $4.50 \pm 0.11$ & $9.4 \pm 2.0$ & $16.1 \pm 2.0$ & 0.9 & 0.2 & 0.3 \\
CVSO 109 & 81.65721 & $3799 \pm 53$ & $4.42 \pm 0.12$ & $<6.0$ & $17.8 \pm 1.7$ & 0.9 & 0.3 & 0.3 \\
CVSO 146 & 92.58387 & $4079 \pm 80$ & $4.70 \pm 0.33$ & $<6.0$ & $16.9 \pm 1.7$ & 0.5 & 0.7 & 0.2 \\
CVSO 165 & 97.58340 & $4040 \pm 73$ & $4.09 \pm 0.24$ & $12.4 \pm 1.0$ & $26.5 \pm 1.8$ & 0.5 & 0.6 & 0.3 \\
CVSO 176 & 85.68282 & $3490 \pm 62$ & $4.21 \pm 0.22$ & $17.0 \pm 7.0$ & $11.1 \pm 1.8$ & 1.4 & 0.5 & 0.6 \\
\hline SO 518 & 85.64623 & $3978 \pm 68$ & $4.42 \pm 0.46$ & $7.0 \pm 12.0$ & $32.3 \pm 2.4$ & 1.0 & 0.9 & 1.3 \\
SO 583 & 85.66222 & $4478 \pm 157$ & $4.26 \pm 0.33$ & $7.0 \pm 8.0$ & $30.9 \pm 1.8$ & 1.0 & 1.3 & 1.3 \\
SO 1153 & 90.70681 & $4086 \pm 63$ & $3.92 \pm 1.02$ & $13.0 \pm 6.0$ & $35.0 \pm 2.2$ & 4.2 & 2.4 & 2.1 \\
\hline
\end{tabular}

Notes. The measured veiling at different wavelengths is reported in the column labelled ' $\mathrm{r}$ ' followed by the wavelength in nm. We estimate uncertainties of $0.1-0.2$ for $r<1.5$ and $0.3-0.4$ for larger values.
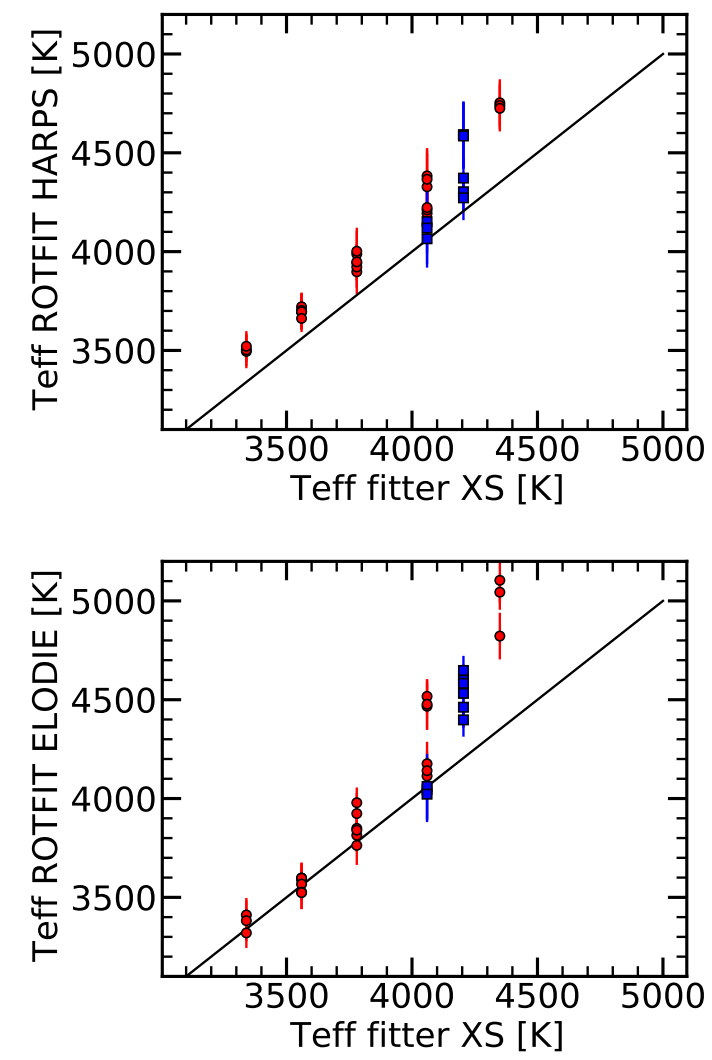

Fig. 6. Effective temperature $\left(T_{\text {eff }}\right)$ derived with ROTFIT on the UVES (red) and ESPRESSO (blue) spectra vs the temperature obtained converting the $\mathrm{SpT}$ from the fit of the X-shooter spectra. Top: ROTFIT results using the HARPS templates, bottom: ROTFIT results using the ELODIE templates.

larger statistical sample during the course of the PENELLOPE program.

Among the values that can be derived with ROTFIT on these spectra, we have a first estimate of metallicity, which is in the range $[\mathrm{Fe} / \mathrm{H}]=[-0.20,+0.05]$ with average values of -0.07 and -0.01 for Ori OB1 and $\sigma$ Ori, respectively. A more detailed analysis of the spectra to determine the values of metallicity and photospheric element abundances is deferred to a future work.
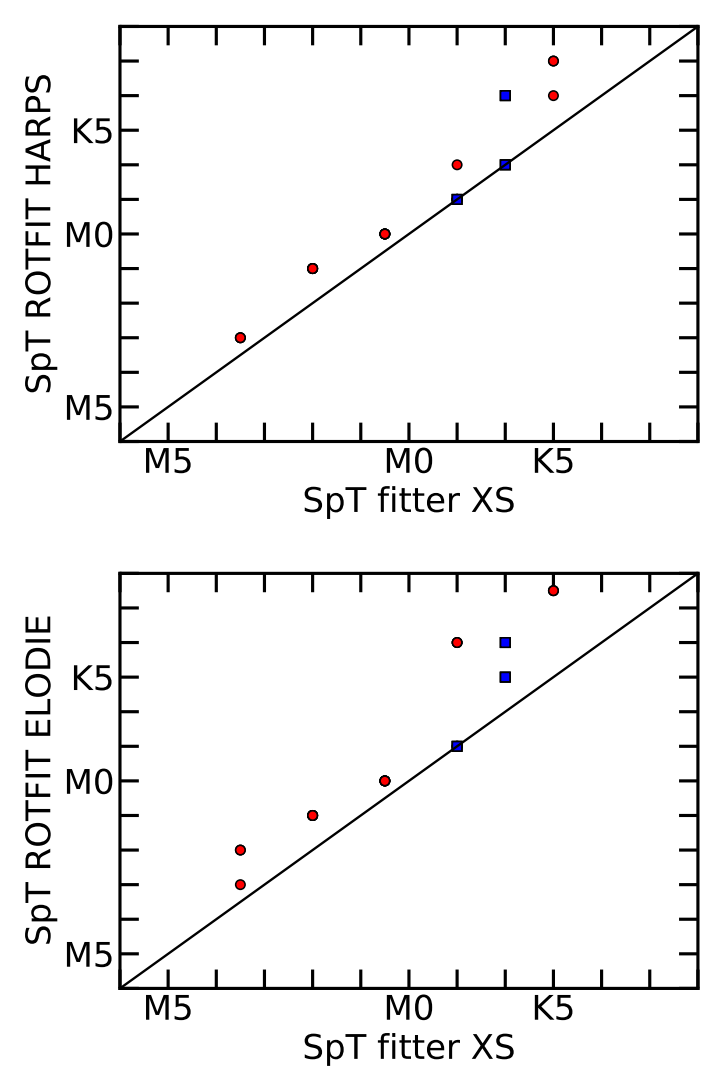

Fig. 7. Spectral type (SpT) derived with ROTFIT on the UVES (red) and ESPRESSO (blue) spectra vs SpT from the fit of the X-shooter spectra. Top: ROTFIT results using the HARPS templates, bottom: ROTFIT results using the ELODIE templates.

\subsection{Variability from high-resolution spectra}

Multiple emission lines are present in our high-resolution spectra (see as an example Figs. G.1-G.13). These lines trace several processes, from accretion (e.g., $\mathrm{H} \alpha, \mathrm{H} \beta, \mathrm{CaIRT}$ ) to winds and outflows (e.g., [OI] $\lambda 6300 \AA$ ). Here, we focus only on the accretion aspect and, in particular, we consider only the $\mathrm{H} \alpha$ line for this analysis. Analyses of the other emission lines are underway and will be presented in companion papers. In addition, we only 


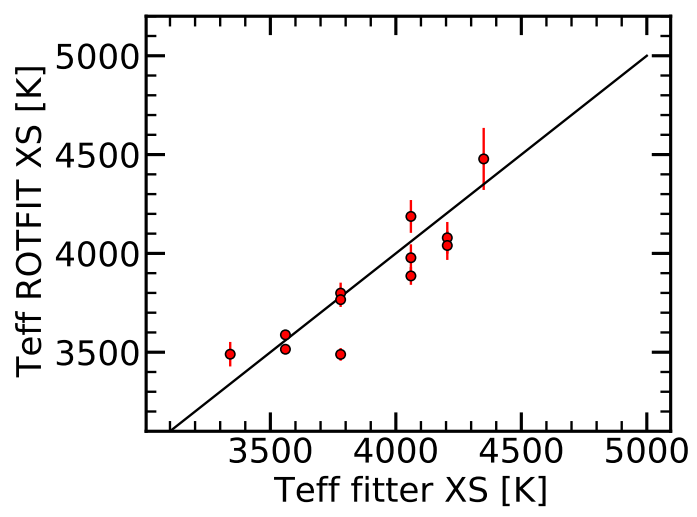

Fig. 8. Effective temperature $\left(T_{\text {eff }}\right)$ derived with ROTFIT on the Xshooter spectra versus the temperature obtained converting the $\mathrm{SpT}$ from the fit of the $\mathrm{X}$-shooter spectra.

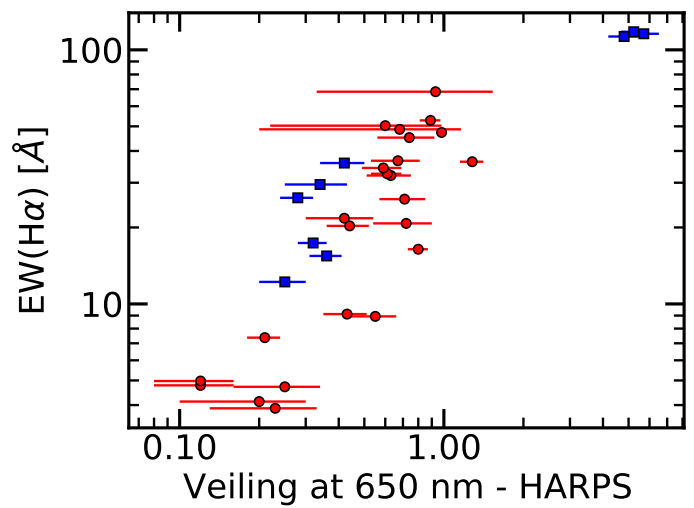

Fig. 9. Veiling measured on the UVES (red) and ESPRESSO (blue) spectra using the HARPS template versus the EW of the $\mathrm{H} \alpha$ line measured on the same spectra. Each target was observed three times.

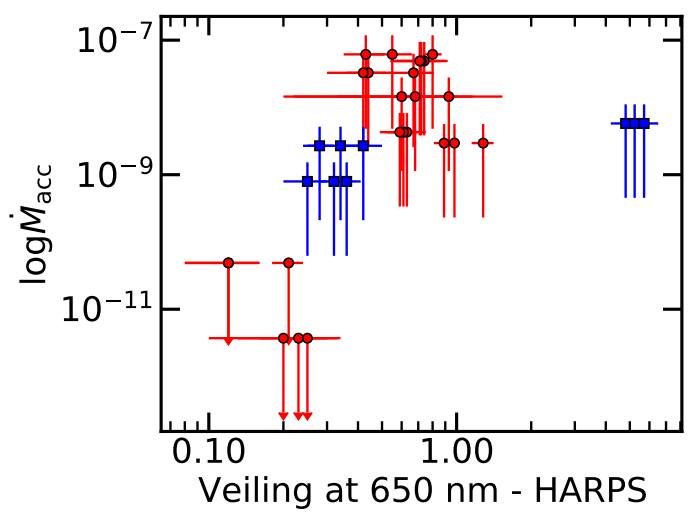

Fig. 10. Veiling measured on the UVES (red) and ESPRESSO (blue) spectra using the HARPS template versus the $\dot{M}_{\text {acc }}$ measured for each target from the X-shooter spectra. Each target was observed three times with UVES or ESPRESSO, and only once with X-shooter.

use the EW of the $\mathrm{H} \alpha$ line in the analysis, as this is sufficient for our purposes, but we note that future works may make use of the wealth of simultaneous photometry to convert these values into line luminosity.

The EW of the $\mathrm{H} \alpha$ line, measured in both the X-shooter and the high-resolution spectra, is shown as a function of time of observations in Figs. B.1 and B.2. The EW values for the two non-accreting targets, CVSO 17 and CVSO 36, are always

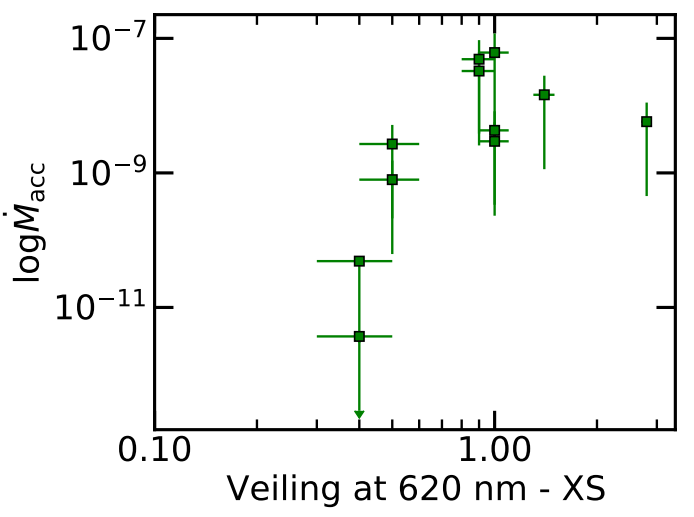

Fig. 11. Veiling measured on the X-shooter spectra vs the $\dot{M}_{\text {acc }}$ measured for each target from the same spectra.

smaller than $10 \AA$, and constant within $\sim 1-2 \AA$ during our observations. Their emission is entirely ascribable to chromospheric emission (e.g., Manara et al. 2013b; White \& Basri 2003). For the other targets, variability of up to a factor $\lesssim 2$ in the EW of the $\mathrm{H} \alpha$ line is observed within the three to four days of observations. Although the EW cannot always be linked to a line flux (e.g., Mendigutía et al. 2013), the relatively small variations in the photometry observed for our targets during the observations imply that the variability in the EW can be related to variations of $L_{\text {acc }}$, and thus $\dot{M}_{\text {acc }}$, by less than a factor of $\sim 3$. This is in line with the typical variability observed on timescales of $\sim$ a week, or less, by other works (e.g., Costigan et al. 2012, 2014; Venuti et al. 2014).

The line profiles, however, can vary substantially, as we can see from the example shown in Fig. 12, and for all the targets in Figs. G.3-G.13. Some of this variability in the line profile is similar to what has been observed by other authors (e.g., Jayawardhana et al. 2006; Biazzo et al. 2012; Fang et al. 2013; Sousa et al. 2016; Bonito et al. 2020). A detailed analysis of the kinematic variations traced by the shape of the emission lines, as well as by the profiles of the forbidden lines, will be subject of future works. Hereafter, we only highlight a few features. The variations observed in the line profiles of CVSO 17 and CVSO 36 (Figs. G.1 and G.2) are modest and consistent with our previous assessment that these lines are almost totally of chromospheric origin.

For CVSO 176, a different line profile shape is present in the $\mathrm{X}$-shooter spectrum, taken two days after the end of the UVES and HST observations, with respect to the profiles of the emission lines in the UVES spectra. In the X-shooter spectra, most lines show a strong red-shifted absorption feature, possibly due to an enhanced accretion event or to a more favorable viewing geometry of the accretion column(s).

For CVSO 109, we observe clear variability in the spectral features across the four epochs. Out of 20 emission lines identified above a $3 \sigma$ detection threshold and common to the $\mathrm{X}$-shooter and UVES coverage, we detect a defined redshifted emission wing in nine $\mathrm{H}$ lines and in the $\mathrm{Ca}$ II $\mathrm{K}$ line. The feature is also prominent in the $\mathrm{Ca}$ II $\mathrm{H}$ line, although unresolved due to proximity with the adjacent $\mathrm{H} 7$ line, and in the Ca II IR triplet, although the latter is not covered by UVES. The redshifted component, detected in the X-shooter and final UVES epoch taken with $\sim 0.5 \mathrm{~h}$ difference, is highly variable, being absent in the first two UVES epochs taken one and two days earlier. Such a temporal feature is indicative of a non-axisymmetric structure in the inner disk, similar to that identified from the spectra of 

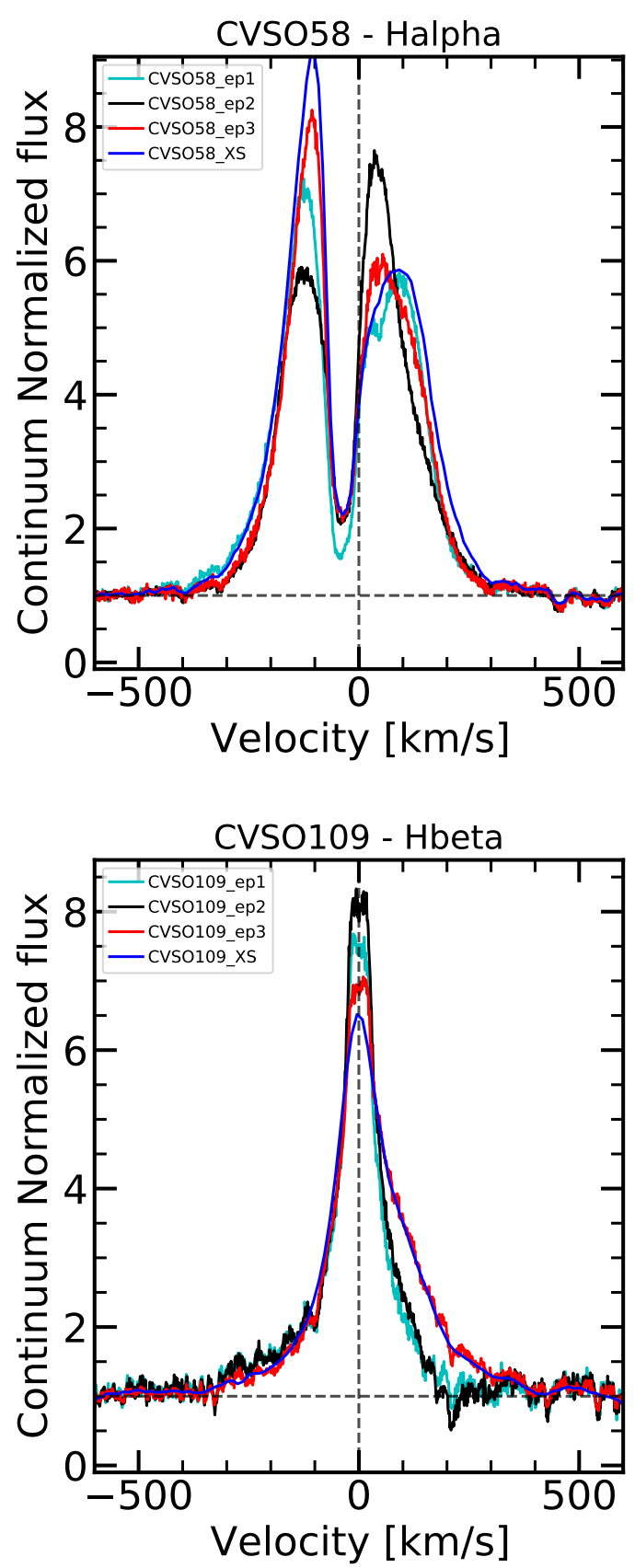

Fig. 12. Example of emission line profiles variability observed with UVES and X-shooter for two targets, as labelled. The time difference between the UVES epochs is always one day, whereas the X-shooter spectrum is obtained on the same night as the last UVES observation.

EX Lupi (Sicilia-Aguilar et al. 2012, 2015). Preliminary analysis of the $\mathrm{H}$ lines reveal that the central velocities of the wings are correlated with the energy of the upper level (Ek) and the transition probability (Aki) of the corresponding emission lines. This, together with the range in velocities, suggests that we are tracing rotating material at different temperatures and densities with varying radii. A more detailed investigation of this target is being carried out using the STAR-MELT analysis package, and will be presented in Campbell-White et al. (in prep.).

CVSO 104 exhibits a peculiar HeI profile with central absorption, as well as unusual variability in the $\mathrm{H} \alpha$ and $\mathrm{H} \beta$ profiles, where the blueshifted emission wings fully disappear at times. This is most probably due to the presence of two unre- solved components of a spectroscopic binary, but could also be related to intermittent ejection of material in either or both of the components.

Finally, variations in the (continuum normalized) line intensity and profile of the [OI] $\lambda 6300 \AA$ and in other forbidden lines is observed in a couple of targets, such as CVSO 107, CVSO 109, CVSO 176, SO 518, and SO 1153. All these targets have been observed with UVES using multiple position angle to orient the slit (see Sect. 2). It might be possible that the variations are related to the presence of (micro-)jets, or to an intrinsic variation of the ejection process. Future detailed analysis of these line profiles is needed, and will be the subject of future works.

\subsection{Variability from photometry}

As described in Sect. 2.3, a ground-based multiband photometric monitoring of our targets was performed from different sites contemporaneously with our observations. As an example, we show in Fig. D. 1 the light curves from OACT, CrAO and AAVSO from mid-November to mid-December for CVSO 109, SO 518, and SO 583. The synthetic photometry made on the flux-calibrated $\mathrm{X}$-shooter spectra is overlaid with purple asterisks. The index $\mathrm{H} \alpha_{18}-\mathrm{H} \alpha_{9}$, which measures the intensity of the $\mathrm{H} \alpha$ emission, has been derived from OACT narrow-band photometry as well as from UVES and X-shooter spectra and is displayed for the two sources in $\sigma$ Ori. The agreement between observed and synthetic photometry is apparent.

Erratic or quasi-periodic variations with an amplitude decreasing with the increasing central wavelength of the band are visible. The largest variation amplitudes $(\approx 1.5-2 \mathrm{mag})$ were observed for CVSO 109 and SO 518 in the $B$ band. Smaller variations are observed for SO 583. We also note, for all these sources, the nearly anticorrelated behaviour of the $\mathrm{H} \alpha$ intensity, which is stronger when the star is fainter and redder, maybe in relation to the location of the accretion funnel with respect to the line of sight. The variability of accretion from photometry and line fluxes will be the subject of a future work.

\section{Discussion}

\subsection{Comparison of $X$-shooter and HST Balmer continua}

One of the main goals of our program is to compare the overall flux calibration and shape of the spectra obtained with VLT/Xshooter and HST/STIS. Figure F.1 show the spectra obtained with the two instruments. Overall, a good agreement is observed, especially in the Balmer continuum slope. The COS and STIS observations of SO 1153 were repeated on 12 February 2021, as the first observations suffered from difficulties in acquiring guide stars and therefore have issues with their wavelength and flux calibrations.

The observations with the smallest time difference between HST/STIS and VLT/X-shooter are for CVSO $58(\Delta t \sim 6 \mathrm{~h})$ and CVSO $109(\Delta t \sim 1.5 \mathrm{~h})$. In these cases, indeed, the difference between the observed flux in the HST/STIS and VLT/Xshooter spectra in the Balmer continuum region is only a factor $\sim 1.3$ and 1 , respectively. However, the slopes of the HST and X-shooter spectra are different at wavelengths longer than $500 \mathrm{~nm}$ for CVSO 109, suggesting either a slightly different extinction toward the object, although probably too large for such a small time difference, or a significant contamination of the X-shooter spectrum by the redder unresolved companion. Despite the larger time difference, other targets also show a good 

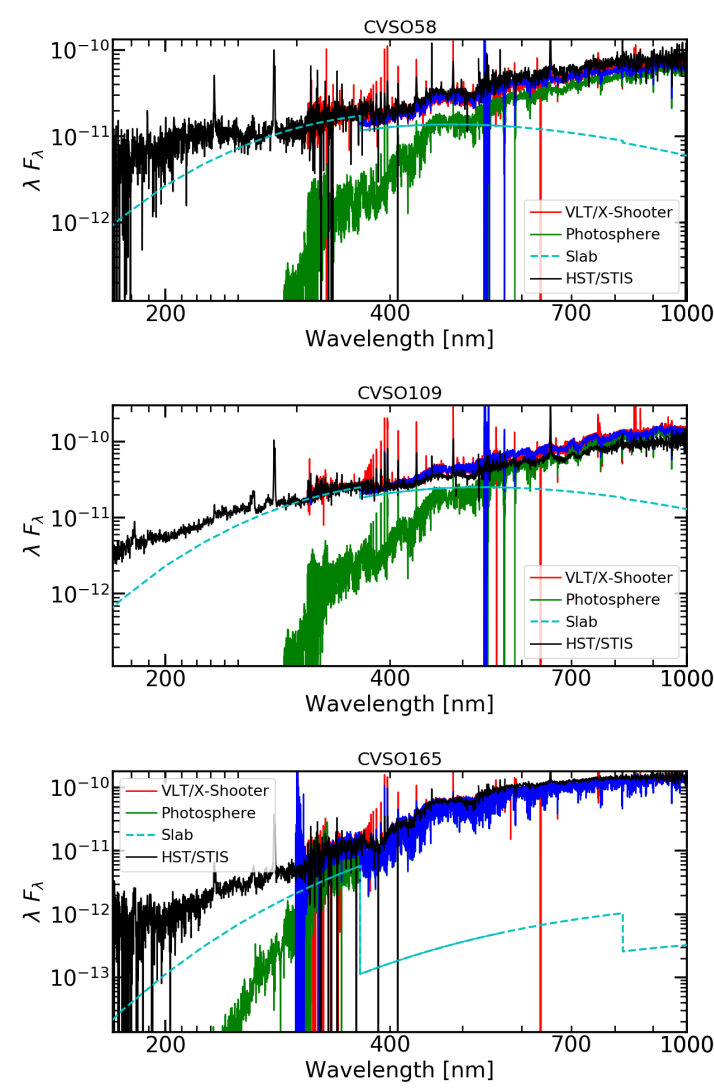

Fig. 13. Comparison of the extrapolation of the results obtained fitting the VLT/X-shooter spectrum (best fit in blue) with the HST spectrum in the UV.

agreement between the X-shooter and HST/STIS spectra, in particular CVSO 107, CVSO 146, CVSO 165, and SO 518.

We focus, in particular, on three targets, CVSO 58, CVSO 109, and CVSO 165, for which the VLT/X-shooter and HST/STIS spectra are observed close in time and have similar shapes, in order to test whether our slab model leading to the best fit of the X-shooter spectra (see Sect. 2.5.1) reproduces the emission from HST at wavelengths shorter than $300 \mathrm{~nm}$. The comparison, performed by scaling the de-reddened HST/STIS spectrum to that of the VLT/X-shooter, is shown in Fig. 13. In general, the HST spectra at $\lambda<300 \mathrm{~nm}$ are brighter than the extrapolation of the slab model in this wavelength range.

To quantify this discrepancy, we compute the excess emission in the HST/STIS spectra relative to the total flux of the slab model, the latter being proportional to $L_{\text {acc }}$. To extract the excess we subtract the photosphere from the X-shooter spectra extended to shorter wavelengths using a BT-Settl synthetic spectrum (Allard et al. 2012) of the same temperature. We also remove the flux of bright emission lines in the HST spectrum. In the cases of CVSO 58 and CVSO 109, the excess emission in the HST spectra with respect to the slab model is $\sim 20 \%$ and $10 \%$ of the total slab model flux, respectively. In both cases, the contribution of the photospheric emission subtracted from the total flux is negligible. This means that the accretion luminosity derived from the slab for these two objects could be underestimated by up to a factor $20 \%$, which is well within the uncertainty of the $L_{\text {acc }}$ values from the fit of the X-shooter spectra (typically 0.2 dex). For CVSO165, instead, a larger factor $\sim 0.5$ is obtained. This target is the one with the lowest value of $L_{\mathrm{acc}} / L_{\star}$ among the accreting targets in our sample. Similarly to Ingleby et al.
(2011) and Alcalá et al. (2019), we see that the excess emission in the UV range could be underestimated more substantially for objects accreting at lower rates and with spectral type earlier than about K3. The latter is a consequence of a lower contrast between the continuum excess emission and the photospheric+chromospheric emission. However, we ought to consider that this target is actually a binary that is unresolved in the VLT observations, but resolved by the HST.

A comparison with the other spectra leads to a relative additional flux in the HST spectra of typically less than $10 \%$ to $\sim 20 \%$, with a maximum of $\sim 25 \%$. With this small sample statistics it is not possible to constrain the origin of this discrepancy. Several factors are at play, including the assumed reddening law (Cardelli et al. 1989, $R_{V}=3.1$ ) and a single temperature to describe the excess emission due to accretion with a slab model. The former affects the shape of the slab model in the UV range more than at optical wavelengths; thus, future works should investigate the kind of reddening law that could reproduce the observed emission at all wavelengths (see also discussion in Alcalá et al. 2019). The single temperature and density assumption is also a simplification of the physical conditions in the accretion region (e.g., Hartmann et al. 2016) and multiple components should be used to better reproduce the shape of the emission at all wavelengths (e.g., Robinson \& Espaillat 2019). However, the small underestimate in the UV of the slab model shown here also indicates that the value of $L_{\text {acc }}$ derived with this procedure is a good estimate of the accretion luminosity of the target.

This kind of analysis, carried out on a larger sample provided by the spectra obtained by the PENELLOPE and ULLYSES programs, will allow us to firmly quantify whether a different reddening law is needed to describe the extinction in the UV part of the spectrum or if the single temperature slab models systemically underestimate the UV flux produced by the accretion process and by how much.

\subsection{Accretion properties of young low-mass stars}

The first set of data obtained by the PENELLOPE program related to young stars in the Orion star-forming region has allowed us to derive stellar and accretion parameters for the 13 targets, two of which were found to be non-accreting. For the eleven accretors, we show that the short-time $(<1$ week) variability of the accretion rate at the time of the observation is small, accounting for a factor $\lesssim 3$ in $L_{\text {acc}}$. This factor is obtained by analyzing the variability of the equivalent width of the $\mathrm{H} \alpha$ line, and is well in line with previous results (e.g., Biazzo et al. 2012; Costigan et al. 2014; Venuti et al. 2014). However, the variability in the kinematics of the accretion-related emission lines is substantial in multiple objects, suggesting that the structure of the accretion flows onto the star is complex and variable, but the overall energy released by the process is relatively constant, at least on these short timescales probed by our observations.

Based on this result, we are confident that the accretion rates measured with our fitting procedure from the broadband $\mathrm{X}$-shooter spectra are a good estimate of the typical accretion rate of the targets at around the time of the observations. Moreover, we have shown that the fit of the X-shooter spectra with a slab model falls short in reproducing the excess emission seen in the UV spectra by $\sim 10 \%$ in most cases. All in all, the typically assumed uncertainties on the values of $L_{\text {acc }}$ of 0.2 dex and on $\dot{M}_{\text {acc }}$ of 0.4 dex from our fitter are in line with both the observed short-term variability and the missing UV flux by the model. 

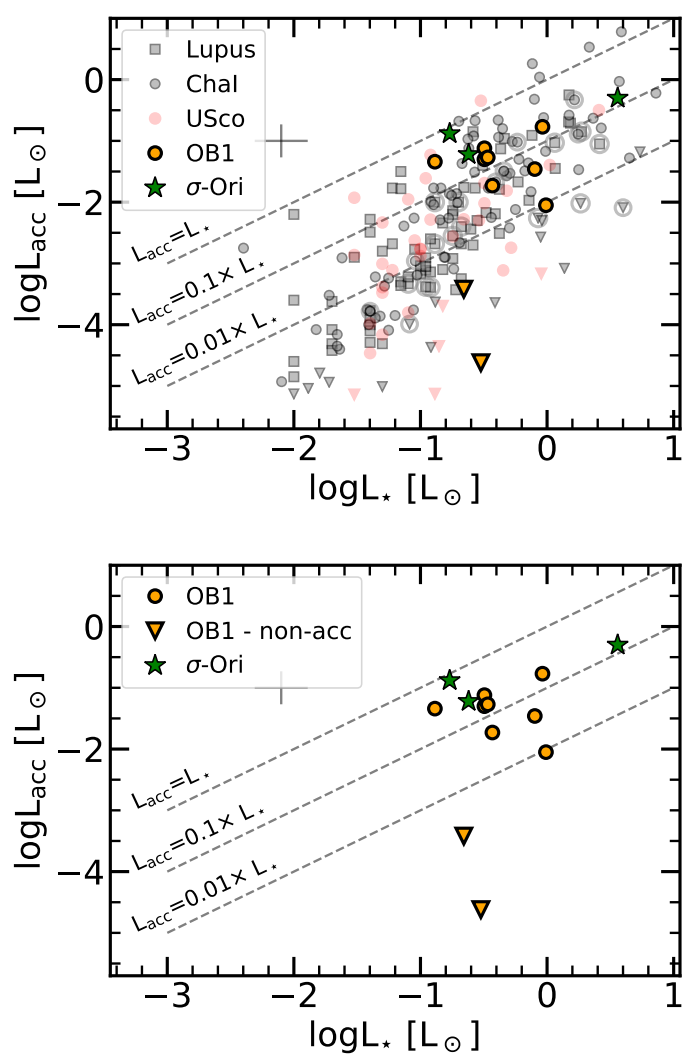

Fig. 14. Relation between the accretion luminosity and stellar luminosity obtained from the X-shooter for the PENELLOPE targets in the Orion region. Top panel: comparison with data obtained with the same technique in the literature, bottom panel: highlights solely the new results from this work.

With the values of $L_{\text {acc }}$ and $\dot{M}_{\text {acc }}$ derived here, we can compare the accretion rates of this population of young stars with previous estimates of accretion rates with the same method in other young star forming regions, in particular, Lupus ( 1-3 Myr old, Alcalá et al. 2014, 2017) rescaled to the Gaia DR2 distances in Alcalá et al. (2019), Chamaeleon I ( 1-3 Myr old, Manara et al. 2016a, 2017b) rescaled to the Gaia DR2 distances in Manara et al. (2019), and Upper Scorpius ( 5-10 Myr old, Manara et al. 2020). The relation between $L_{\mathrm{acc}}$ and $L_{\star}$ for the targets in these regions is shown in Fig. 14. In line with previous results, the data of accreting targets, including those presented here, follow the same $L_{\text {acc }}-L_{\star}$ relation as target in other star-forming regions with younger and older ages. No dependence on age or location is observed. The upper limits on $L_{\text {acc }}$ derived for the two non-accreting targets are found at values of $L_{\text {acc }} / L_{\star}<0.01$, in line with other non-accreting systems observed in young regions.

The values of $\dot{M}_{\text {acc }}$ vs. $M_{\star}$ for the targets in Orion and in the other young regions is shown in Fig. 15. Also in this case, the data presented here on the Orion targets populate the same part of the parameter space as targets observed in other star-forming regions. The observed scatter of $\dot{M}_{\text {acc }}$ at a given value of $M_{\star}$ is large even in this small sample, $\sim 2$ dex when considering only the accreting objects. This scatter is much larger than the variability we observe on timescales of a few days, which is thought to be the maximum observed variability on timescales of years (Costigan et al. 2012, 2014). The origin of the scatter should be then ascribed to real differences in the accretion properties of targets in a single star-forming region, possibly due to dif-
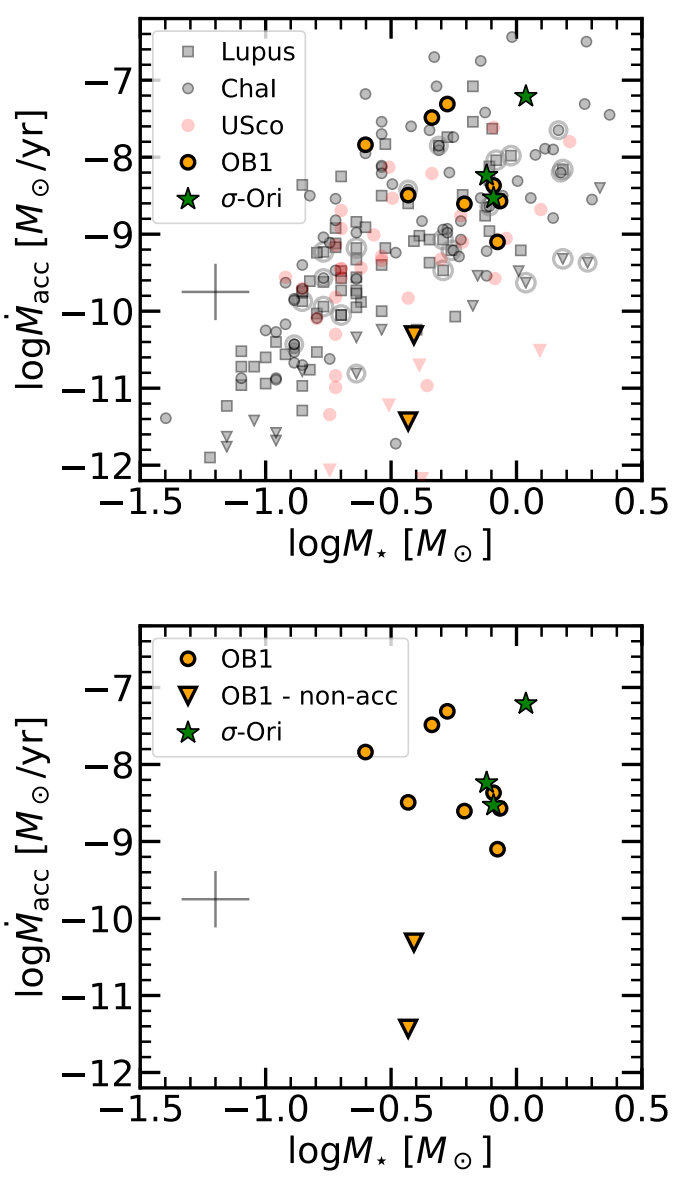

Fig. 15. Relation between the mass accretion rates and stellar mass obtained from the X-shooter for the PENELLOPE targets in the Orion region. Top panel: comparison with data obtained with the same technique in the literature, bottom panel: highlights solely the new results from this work.

ferent disk evolutionary stages (e.g., Najita et al. 2007, 2015; Manara et al. 2014) or properties of the magnetic field (e.g., Gregory et al. 2006) or of the details of the star-disk interaction, or even of the surroundings, such as the presence of companions (e.g., Rosotti \& Clarke 2018). A detailed analysis of the observed emission lines for these targets will help to understand the origin of these differences. The non-accreting targets observed here have upper limits on $\dot{M}_{\text {acc }}$ much smaller than the typical $\dot{M}_{\text {acc }}$ observed for stars of the same stellar mass. This is in line with what is observed in other star-forming regions and may also indicate rapid dispersal of the (inner) disk as processes such as photoevaporation take over (e.g., Ercolano \& Pascucci 2017).

Overall, we do not see noticeable differences between the accretion rates measured in the 13 targets in the Orion OB 1 and $\sigma$-Ori regions with respect to what is observed in other young star-forming regions with similar techniques. This result is in agreement with the findings of Manara et al. (2020), Venuti et al. (2019), Rugel et al. (2018), and Ingleby et al. (2014), among others, who found that the accretion rates of still accreting objects at ages of $>3-5 \mathrm{Myr}$ are still comparable to the accretion rates of younger stars. However, this result is still puzzling as these high accretion rates are difficult to reconcile with the current framework to describe disk evolution (e.g., Ingleby et al. 2014; Manara et al. 2020). Larger samples can help to statistically study the differences between the populations of various regions 
and provide the necessary data for constraining models based on observations.

\section{Conclusions}

In this paper, we present the survey strategy and a set of initial results from the PENELLOPE Large Program aimed at obtaining observations with VLT/X-shooter, and VLT/ESPRESSO or VLT/UVES, contemporaneously with the HST STIS and COS observations of the public ULLYSES survey. Complemented with contemporaneous photometry, these programs will provide an unprecedented view of the accretion and ejection processes in young stars.

We showcase the observing strategy, data reduction procedure, and data analysis tools on a sample of ten targets in the Orion OB1 association and further three targets in the $\sigma$-Orionis region, observed with HST and VLT in November and December 2020. We have presented the contemporaneous multi-band photometry obtained at OACT and show the good agreement between the absolute flux-calibrated X-shooter spectra and this photometric data.

We show that the agreement between the temperature and spectral type estimates obtained with the two methods used here on the X-shooter spectra and on the high-resolution spectra is consistent within a sub-class. We then found that the veiling measured on the high-resolution spectra with ROTFIT correlates with the $\mathrm{EW}$ of the $\mathrm{H} \alpha$ line measured on the same spectra, whereas no strong correlation is found between the veiling and $\dot{M}_{\text {acc }}$.

We were able to constrain the short-term $(<1$ week) variability of the accretion luminosity for these targets to be a factor $\$ 3$, in line with previous works (e.g., Cody et al. 2014, 2017; Venuti et al. 2014; Costigan et al. 2012, 2014). Future works on the dataset from this program will also allow for the long-term (a few years) variability of these targets to be constrained thanks to the availability of previous spectra obtained with the same or with different instruments. On the other hand, the profiles of accretion and ejection tracing lines vary substantially between the various epochs, suggesting that the structure of the accretion flow is complex and variabile, whereas the energy released is relatively constant. The data from this program will allow for detailed analyses of the structure of the accretion and ejection emitting regions for all the targets to further understand the accretion and ejection processes as well as their interplay.

The first comparison of the HST/STIS and X-shooter spectra on a limited number of targets suggests that the slab models used to fit the X-shooter spectra underestimate the UV emission seen in the HST spectra by $\sim 10 \%$, and usually less than $\sim 20 \%$, in the targets observed here. Several causes can be ascribed for this discrepancy, such as an inappropriate extinction curve in the UV part of the spectrum, or a real shortcoming of a single temperature model to reproduce the excess emission in the UV. These hypotheses will be investigated in future works with a larger sample of contemporaneous HST/STIS and X-shooter spectra. Nevertheless, these findings demonstrate the general validity of the accretion rates measured with $\mathrm{X}$-shooter on these spectra, and that the reported uncertainties of $\sim 0.2 \mathrm{dex}$ on $L_{\text {acc }}$ and $\sim 0.4$ dex on $\dot{M}_{\text {acc }}$ are reasonable. Finally, we compared the relation between the accretion rate and stellar mass, showing similar values of $\dot{M}_{\text {acc }}$ and similar spread of values at any $M_{\star}$ as in previous surveys.

The VLT/PENELLOPE program will continue to obtain data alongside the HST/ULLYSES program for the next $\lesssim 2$ years, providing a public dataset that will be key to creating a coherent and comprehensive view of the accretion process and its impact on the evolution of disks in nearby star-forming regions. This dataset will also be key to constraining other processes not showcased in this work, such as the ejection of matter in young stellar objects, probed by the high-resolution multi-epoch spectra available from this program. We invite the community to make use of these public datasets, acknowledging the effort of the group of people who initiated this effort, and with an open desire to enhance our knowledge.

Acknowledgements. We thank the referee for the constructive report. We thank the ESO support staff for the help in the preparation of the observations, in the scheduling, and for carrying out the observations at Paranal. We also thank the ESO staff, in particular John Pritchard and Lodovico Coccato, for implementing the Molecfit workflow for ESPRESSO and making it available to us. We thank Antonella Natta for insightful discussions. Based on observations obtained with the NASA/ESA Hubble Space Telescope, retrieved from the Mikulski Archive for Space Telescopes (MAST) at the Space Telescope Science Institute (STScI). STScI is operated by the Association of Universities for Research in Astronomy, Inc. under NASA contract NAS 5-26555. This work is supported in part by the ODYSSEUS team (HST AR-16129), https://sites.bu.edu/odysseus/. This project has received funding from the European Union's Horizon 2020 research and innovation programme under the Marie Sklodowska-Curie grant agreement No. 823823 (DUSTBUSTERS). This research received financial support from the project PRIN-INAF-MAINSTREAM "Protoplanetary disks seen through the eyes of new-generation instruments" (CUPC54I19000600005) and PRIN-INAF 2019 "Spectroscopically Tracing the Disk Dispersal Evolution". This work was partly supported by the Deutsche Forschungs-Gemeinschaft (DFG, German Research Foundation) - Ref no. FOR 2634/1 TE 1024/1-1. This work has made use of data from the European Space Agency (ESA) mission Gaia (https://www.cosmos.esa.int/gaia), processed by the Gaia Data Processing and Analysis Consortium (DPAC, https://www.cosmos.esa.int/ web/gaia/dpac/consortium). Funding for the DPAC has been provided by national institutions, in particular the institutions participating in the Gaia Multilateral Agreement. JCW and ASA are supported by the STFC grant number ST/S000399/1 ("The Planet-Disk Connection: Accretion, Disk Structure, and Planet Formation"). ACG and PMG acknowledge funding from the European Research Council under Advanced Grant No. 743029, Ejection, Accretion Structures in YSOs (EASY). BE acknowledges support from the DFG Research Unit "Transition Disks" (FOR 2634/1, ER 685/8-1) and from the DFG cluster of excellence Origin and Structure of the Universe (http://www . universe-cluster.de/). R.G.L. acknowledges support by Science Foundation Ireland under Grant No. 18/SIRG/5597. This project has received funding from the European Research Council (ERC) under the European Union's Horizon 2020 research and innovation programme under grant agreement No. 716155 (SACCRED). L.V. acknowledges support by an appointment to the NASA Postdoctoral Program at the NASA Ames Research Center, administered by Universities Space Research Association under contract with NASA. G.R. acknowledges support from the Netherlands Organisation for Scientific Research (NWO, program number 016.Veni.192.233) and from an STFC Ernest Rutherford Fellowship (grant number ST/T003855/1). J.H. acknowledges support from the National Research Council of México (CONACyT) project No. 86372 and the PAPIIT UNAM project IA102921.

\section{References}

Alam, S., Albareti, F. D., Allende Prieto, C., et al. 2015, ApJS, 219, 12 Alcalá, J. M., Natta, A., Manara, C. F., et al. 2014, A\&A, 561, A2 Alcalá, J. M., Manara, C. F., Natta, A., et al. 2017, A\&A, 600, A20 Alcalá, J. M., Manara, C. F., France, K., et al. 2019, A\&A, 629, A108 Alexander, R. D., Clarke, C. J., \& Pringle, J. E. 2006, MNRAS, 369, 229 Alexander, R., Pascucci, I., Andrews, S., Armitage, P., \& Cieza, L. 2014, Protostars and Planets VI (Tucson: University of Arizona Press), 475 Allard, F., Homeier, D., \& Freytag, B. 2012, Phil. Trans. R. Soc. Lond. Ser. A, 370,2765

Ansdell, M., Williams, J. P., Manara, C. F., et al. 2017, AJ, 153, 240 Antoniucci, S., García López, R., Nisini, B., et al. 2014, A\&A, 572, A62 Arulanantham, N., France, K., Hoadley, K., et al. 2018, ApJ, 855, 98 Arulanantham, N., France, K., Cazzoletti, P., et al. 2020, AJ, 159, 168 Bai, X.-N. 2016, ApJ, 821, 80

Bailer-Jones, C. A. L. 2015, PASP, 127, 994

Bailer-Jones, C. A. L., Rybizki, J., Fouesneau, M., Demleitner, M., \& Andrae, R. 2021, AJ, 161, 147

Ballabio, G., Alexander, R. D., \& Clarke, C. J. 2020, MNRAS, 496, 2932 Ballester, P., Modigliani, A., Boitquin, O., et al. 2000, The Messenger, 101, 31 Banzatti, A., Pascucci, I., Edwards, S., et al. 2019, ApJ, 870, 76 
Baraffe, I., Homeier, D., Allard, F., \& Chabrier, G. 2015, A\&A, 577, A42 Basri, G., \& Batalha, C. 1990, ApJ, 363, 654

Biazzo, K., Alcalá, J. M., Covino, E., et al. 2012, A\&A, 547, A104

Blaauw, A. 1964, ARA\&A, 2, 213

Bonito, R., Prisinzano, L., Venuti, L., et al. 2020, A\&A, 642, A56

Bouvier, J., Alencar, S. H. P., Harries, T. J., Johns-Krull, C. M., \& Romanova, M. M. 2007, in Protostars and Planets V, eds. B. Reipurth, D. Jewitt, \& K Keil, 479

Brewer, J. M., Fischer, D. A., Valenti, J. A., \& Piskunov, N. 2016, ApJS, 225, 32

Briceño, C., Vivas, A. K., Calvet, N., et al. 2001, Science, 291, 93

Briceño, C., Calvet, N., Hernández, J., et al. 2005, AJ, 129, 907

Briceño, C., Calvet, N., Hernández, J., et al. 2019, AJ, 157, 85

Caballero, J. A. 2014, The Observatory, 134, 273

Caballero, J. A. 2018, Res. Notes Am. Astron. Soc., 2, 25

Caballero, J. A., de Burgos, A., Alonso-Floriano, F. J., et al. 2019, A\&A, 629, A114

Calvet, N., Muzerolle, J., Briceño, C., et al. 2004, AJ, 128, 1294

Cardelli, J. A., Clayton, G. C., \& Mathis, J. S. 1989, ApJ, 345, 245

Chambers, K. C., Magnier, E. A., Metcalfe, N., et al. 2016, ArXiv e-prints [arXiv:1612.05560]

Clarke, C. J., \& Pringle, J. E. 2006, MNRAS, 370, L10

Cody, A. M., \& Hillenbrand, L. A. 2010, ApJS, 191, 389

Cody, A. M., Stauffer, J., Baglin, A., et al. 2014, AJ, 147, 82

Cody, A. M., Hillenbrand, L. A., David, T. J., et al. 2017, ApJ, 836, 41

Costigan, G., Scholz, A., Stelzer, B., et al. 2012, MNRAS, 427, 1344

Costigan, G., Vink, J. S., Scholz, A., Ray, T., \& Testi, L. 2014, MNRAS, 440, 3444

Dekker, H., D’Odorico, S., Kaufer, A., Delabre, B., \& Kotzlowski, H. 2000, in Optical and IR Telescope Instrumentation and Detectors, eds. M. Iye, \& A. F Moorwood, SPIE Conf. Ser., 4008, 534

DeSouza, A. L., \& Basu, S. 2017, New Astron., 51, 113

Dullemond, C. P., Natta, A., \& Testi, L. 2006, ApJ, 645, L69

Ercolano, B., \& Owen, J. E. 2016, MNRAS, 460, 3472

Ercolano, B., \& Pascucci, I. 2017, R. Soc. Open Sci., 4, 170114

Ercolano, B., Mayr, D., Owen, J. E., Rosotti, G., \& Manara, C. F. 2014, MNRAS 439, 256

Fang, M., Kim, J. S., van Boekel, R., et al. 2013, ApJS, 207, 5

Fang, M., Pascucci, I., Edwards, S., et al. 2018, ApJ, 868, 28

Ferreira, J., Dougados, C., \& Cabrit, S. 2006, A\&A, 453, 785

Fischer, W. J., Megeath, S. T., Furlan, E., et al. 2017, ApJ, 840, 69

Frank, A., Ray, T. P., Cabrit, S., et al. 2014, Protostars and Planets VI (Tucson: University of Arizona Press), 451

Frasca, A., Freire Ferrero, R., Marilli, E., \& Catalano, S. 2000, A\&A, 364, 179

Frasca, A., Biazzo, K., Lanzafame, A. C., et al. 2015, A\&A, 575, A4

Frasca, A., Biazzo, K., Alcalá, J. M., et al. 2017, A\&A, 602, A33

Frasca, A., Montes, D., Alcalà, J. M., Klutsch, A., \& Guillout, P. 2018, Acta Astron., 68, 403

Freudling, W., Romaniello, M., Bramich, D. M., et al. 2013, A\&A, 559, A96

Gagné, J., Plavchan, P., Gao, P., et al. 2016, ApJ, 822, 40

Gaia Collaboration (Prusti, T., et al.) 2016, A\&A, 595, A1

Gaia Collaboration (Brown, A. G. A., et al.) 2021, A\&A, 649, A1

García Pérez, A. E., Allende Prieto, C., Holtzman, J. A., et al. 2016, AJ, 151, 144

Giannini, T., Nisini, B., Antoniucci, S., et al. 2019, A\&A, 631, A44

Glebocki, R., \& Gnacinski, P. 2005, VizieR Online Data Catalog: III/244

Grandjean, A., Lagrange, A. M., Keppler, M., et al. 2020, A\&A, 633, A44

Gregory, S. G., Jardine, M., Simpson, I., \& Donati, J. F. 2006, MNRAS, 371, 999

Hartigan, P., Edwards, S., \& Ghandour, L. 1995, ApJ, 452, 736

Hartmann, L., Calvet, N., Gullbring, E., \& D’Alessio, P. 1998, ApJ, 495, 385

Hartmann, L., D’Alessio, P., Calvet, N., \& Muzerolle, J. 2006, ApJ, 648, 484

Hartmann, L., Herczeg, G., \& Calvet, N. 2016, ARA\&A, 54, 135

Henden, A. A., Levine, S., Terrell, D., et al. 2018, Am. Astron. Soc. Meet. Abstr. 232, 223.06

Herczeg, G. J., \& Hillenbrand, L. A. 2008, ApJ, 681, 594

Herczeg, G. J., \& Hillenbrand, L. A. 2014, ApJ, 786, 97

Hernández, J., Hartmann, L., Megeath, T., et al. 2007a, ApJ, 662, 1067

Hernández, J., Calvet, N., Briceño, C., et al. 2007b, ApJ, 671, 1784

Hernández, J., Calvet, N., Perez, A., et al. 2014, ApJ, 794, 36

Hillenbrand, L. A., Strom, S. E., Vrba, F. J., \& Keene, J. 1992, ApJ, 397, 613

Hojjatpanah, S., Figueira, P., Santos, N. C., et al. 2019, A\&A, 629, A80

Houdebine, E. R., Mullan, D. J., Paletou, F., \& Gebran, M. 2016, ApJ, 822, 97

Ingleby, L., Calvet, N., Bergin, E., et al. 2009, ApJ, 703, L137

Ingleby, L., Calvet, N., Hernández, J., et al. 2011, AJ, 141, 127

Ingleby, L., Calvet, N., Hernández, J., et al. 2014, ApJ, 790, 47

Jayawardhana, R., Coffey, J., Scholz, A., Brandeker, A., \& van Kerkwijk, M. H 2006, ApJ, 648, 1206

Jeffers, S. V., Schöfer, P., Lamert, A., et al. 2018, A\&A, 614, A76
Jeffries, R. D., Maxted, P. F. L., Oliveira, J. M., \& Naylor, T. 2006, MNRAS, 371, L6

Johns, C. M., \& Basri, G. 1995a, AJ, 109, 2800

Johns, C. M., \& Basri, G. 1995b, ApJ, 449, 341

Jones, M. G., Pringle, J. E., \& Alexander, R. D. 2012, MNRAS, 419, 925

Kausch, W., Noll, S., Smette, A., et al. 2015, A\&A, 576, A78

Kenyon, S. J., \& Hartmann, L. 1995, ApJS, 101, 117

Kóspál, Á., Szabó, Z. M., Ábrahám, P., et al. 2020, ApJ, 889, 148

Kounkel, M., Covey, K., Suárez, G., et al. 2018, AJ, 156, 84

Kurosawa, R., Harries, T. J., \& Symington, N. H. 2006, MNRAS, 370, 580

Landolt, A. U. 2009, AJ, 137, 4186

Lindgren, S., \& Heiter, U. 2017, A\&A, 604, A97

Lindegren, L., Bastian, U., Biermann, M., et al. 2021, A\&A, 649, A4

Lodato, G., Scardoni, C. E., Manara, C. F., \& Testi, L. 2017, MNRAS, 472, 4700

Luck, R. E. 2017, AJ, 153, 21

Luck, R. E. 2018, AJ, 155, 111

Luhman, K. L., Stauffer, J. R., Muench, A. A., et al. 2003, ApJ, 593, 1093

Lynden-Bell, D., \& Pringle, J. E. 1974, MNRAS, 168, 603

Manara, C. F., Robberto, M., Da Rio, N., et al. 2012, ApJ, 755, 154

Manara, C. F., Beccari, G., Da Rio, N., et al. 2013a, A\&A, 558, A114

Manara, C. F., Testi, L., Rigliaco, E., et al. 2013b, A\&A, 551, A107

Manara, C. F., Testi, L., Natta, A., et al. 2014, A\&A, 568, A18

Manara, C. F., Fedele, D., Herczeg, G. J., \& Teixeira, P. S. 2016a, A\&A, 585, A136

Manara, C. F., Rosotti, G., Testi, L., et al. 2016b, A\&A, 591, L3

Manara, C. F., Testi, L., Herczeg, G. J., et al. 2017a, A\&A, 604, A127

Manara, C. F., Frasca, A., Alcalá, J. M., et al. 2017b, A\&A, 605, A86

Manara, C. F., Mordasini, C., Testi, L., et al. 2019, A\&A, 631, L2

Manara, C. F., Natta, A., Rosotti, G. P., et al. 2020, A\&A, 639, A58

Maucó, K., Hernández, J., Calvet, N., et al. 2016, ApJ, 829, 38

Maucó, K., Briceño, C., Calvet, N., et al. 2018, ApJ, 859, 1

McDonald, I., Zijlstra, A. A., \& Watson, R. A. 2017, MNRAS, 471, 770

McGinnis, P., Dougados, C., Alencar, S. H. P., Bouvier, J., \& Cabrit, S. 2018, A\&A, 620, A87

Mendigutía, I., Brittain, S., Eiroa, C., et al. 2013, ApJ, 776, 44

Mishenina, T. V., Soubiran, C., Kovtyukh, V. V., Katsova, M. M., \& Livshits, M. A. 2012, A\&A, 547, A106

Modigliani, A., Goldoni, P., Royer, F., et al. 2010, in Observatory Operations: Strategies, Processes, and Systems III, eds. D. R. Silva, A. B. Peck, \& B. T. Soifer, SPIE Conf. Ser., 7737, 773728

Mohanty, S., Jayawardhana, R., \& Basri, G. 2005, ApJ, 626, 498

Montes, D., González-Peinado, R., Tabernero, H. M., et al. 2018, MNRAS, 479, 1332

Morbidelli, A., \& Raymond, S. N. 2016, J. Geophys. Res., 121, 1962

Moultaka, J., Ilovaisky, S. A., Prugniel, P., \& Soubiran, C. 2004, PASP, 116, 693

Mulders, G. D., Pascucci, I., Manara, C. F., et al. 2017, ApJ, 847, 31

Muzerolle, J., Calvet, N., \& Hartmann, L. 1998, ApJ, 492, 743

Muzerolle, J., Calvet, N., \& Hartmann, L. 2001, ApJ, 550, 944

Muzerolle, J., Hillenbrand, L., Calvet, N., Briceño, C., \& Hartmann, L. 2003, ApJ, 592, 266

Najita, J. R., Strom, S. E., \& Muzerolle, J. 2007, MNRAS, 378, 369

Najita, J. R., Andrews, S. M., \& Muzerolle, J. 2015, MNRAS, 450, 3559

Natta, A., Testi, L., \& Randich, S. 2006, A\&A, 452, 245

Natta, A., Testi, L., Alcalá, J. M., et al. 2014, A\&A, 569, A5

Nisini, B., Antoniucci, S., Alcalá, J. M., et al. 2018, A\&A, 609, A87

Padoan, P., Kritsuk, A., Norman, M. L., \& Nordlund, Å. 2005, ApJ, 622, L61

Passegger, V. M., Wende-von Berg, S., \& Reiners, A. 2016, A\&A, 587, A19

Passegger, V. M., Reiners, A., Jeffers, S. V., et al. 2018, A\&A, 615, A6

Passegger, V. M., Schweitzer, A., Shulyak, D., et al. 2019, A\&A, 627, A161

Pecaut, M. J., \& Mamajek, E. E. 2013, ApJS, 208, 9

Pepe, F., Cristiani, S., Rebolo, R., et al. 2021, A\&A, 645, A96

Pérez-Blanco, A., Maucó, K., Hernández, J., et al. 2018, ApJ, 867, 116

Press, W. H., Teukolsky, S. A., Vetterling, W. T., \& Flannery, B. P. 1992, Numerical Recipes in FORTRAN. The Art of Scientific Computing (Cambridge: Cambridge University Press)

Pringle, J. E. 1981, ARA\&A, 19, 137

Proffitt, C. R., Roman-Duval, J., Taylor, J. M., et al. 2021, Res. Notes Am. Astron. Soc., 5, 36

Pudritz, R. E., \& Ray, T. P. 2019, Front. Astron. Space Sci., 6, 54

Rajpurohit, A. S., Allard, F., Teixeira, G. D. C., et al. 2018, A\&A, 610, A19

Reiners, A., Joshi, N., \& Goldman, B. 2012, AJ, 143, 93

Reiners, A., Zechmeister, M., Caballero, J. A., et al. 2018, A\&A, 612, A49

Rich, E. A., Wisniewski, J. P., McElwain, M. W., et al. 2017, MNRAS, 472, 1736

Ricker, G. R., Winn, J. N., Vanderspeck, R., et al. 2015, J. Astron. Telesc. Instrum. Syst., 1, 014003

Rigliaco, E., Natta, A., Randich, S., Testi, L., \& Biazzo, K. 2011, A\&A, 525, A47 
Rigliaco, E., Natta, A., Testi, L., et al. 2012, A\&A, 548, A56

Rigliaco, E., Pascucci, I., Gorti, U., Edwards, S., \& Hollenbach, D. 2013, ApJ, 772,60

Robinson, C. E., \& Espaillat, C. C. 2019, ApJ, 874, 129

Rojas-Ayala, B., Covey, K. R., Muirhead, P. S., \& Lloyd, J. P. 2012, ApJ, 748, 93

Roman-Duval, J., Proffitt, C. R., Taylor, J. M., et al. 2020, Res. Notes Am. Astron. Soc. 4, 205

Rosotti, G. P., \& Clarke, C. J. 2018, MNRAS, 473, 5630

Rosotti, G. P., Clarke, C. J., Manara, C. F., \& Facchini, S. 2017, MNRAS, 468, 1631

Rugel, M., Fedele, D., \& Herczeg, G. 2018, A\&A, 609, A70

Schweitzer, A., Passegger, V. M., Cifuentes, C., et al. 2019, A\&A, 625, A68

Sellek, A. D., Booth, R. A., \& Clarke, C. J. 2020a, MNRAS, 492, 1279

Sellek, A. D., Booth, R. A., \& Clarke, C. J. 2020b, MNRAS, 498, 2845

Sicilia-Aguilar, A., Henning, T., \& Hartmann, L. W. 2010, ApJ, 710, 597

Sicilia-Aguilar, A., Kóspál, Á., Setiawan, J., et al. 2012, A\&A, 544, A93

Sicilia-Aguilar, A., Fang, M., Roccatagliata, V., et al. 2015, A\&A, 580, A82

Siess, L., Dufour, E., \& Forestini, M. 2000, A\&A, 358, 593

Simon, M. N., Pascucci, I., Edwards, S., et al. 2016, ApJ, 831, 169

Siwak, M., Ogloza, W., Moffat, A. F. J., et al. 2018, MNRAS, 478, 758

Smette, A., Sana, H., Noll, S., et al. 2015, A\&A, 576, A77

Somigliana, A., Toci, C., Lodato, G., Rosotti, G., \& Manara, C. F. 2020, MNRAS, 492, 1120

Sousa, A. P., Alencar, S. H. P., Bouvier, J., et al. 2016, A\&A, 586, A47

Stauffer, J., Cody, A. M., Baglin, A., et al. 2014, AJ, 147, 83

Stetson, P. B. 2000, PASP, 112, 925

Thanathibodee, T., Calvet, N., Herczeg, G., et al. 2018, ApJ, 861, 73

Thanathibodee, T., Calvet, N., Muzerolle, J., et al. 2019, ApJ, 884, 86

Tokovinin, A., Petr-Gotzens, M. G., \& Briceño, C. 2020, AJ, 160, 268

Torres, C. A. O., Quast, G. R., da Silva, L., et al. 2006, A\&A, 460, 695

Valenti, J. A., \& Fischer, D. A. 2005, ApJS, 159, 141

Venuti, L., Bouvier, J., Flaccomio, E., et al. 2014, A\&A, 570, A82

Venuti, L., Stelzer, B., Alcalá, J. M., et al. 2019, A\&A, 632, A46

Vernet, J., Dekker, H., D’Odorico, S., et al. 2011, A\&A, 536, A105

Vorobyov, E. I., \& Basu, S. 2009, ApJ, 703, 922

Walter, F. M., Wolk, S. J., Freyberg, M., \& Schmitt, J. H. M. M. 1997, Mem. Soc. Astron. It., 68, 1081

Weber, M. L., Ercolano, B., Picogna, G., Hartmann, L., \& Rodenkirch, P. J. 2020, MNRAS, 496, 223

White, R. J., \& Basri, G. 2003, ApJ, 582, 1109

Winter, A. J., Clarke, C. J., Rosotti, G., et al. 2018, MNRAS, 478, 2700

Woolf, V. M., \& Wallerstein, G. 2005, MNRAS, 356, 963

Yee, S. W., Petigura, E. A., \& von Braun, K. 2017, ApJ, 836, 77

Zboril, M., \& Byrne, P. B. 1998, MNRAS, 299, 753

1 European Southern Observatory, Karl-Schwarzschild-Strasse 2, 85748 Garching bei München, Germany e-mail: cmanara@eso.org

${ }^{2}$ INAF - Osservatorio Astrofisico di Catania, Via S. Sofia, 78, 95123 Catania, Italy

${ }^{3}$ NASA Ames Research Center, Moffett Blvd, Mountain View, CA 94035, USA

${ }^{4}$ Konkoly Observatory, Research Centre for Astronomy and Earth Sciences, Eötvös Loránd Research Network (ELKH), KonkolyThege Miklós út 15-17, 1121 Budapest, Hungary

5 Kavli Institute for Astronomy and Astrophysics, Peking University, Yiheyuan 5, Haidian Qu 100871, Beijing, PR China

${ }^{6}$ Department of Astronomy, University of Michigan, 1085 S. University Ave., Ann Arbor, MI 48109, USA

7 Instituto de Astronomía, UNAM, Campus Ensenada, Carretera Tijuana-Ensenada km 103, 22860 Ensenada, BC, Mexico

8 INAF - Osservatorio Astronomico di Roma, Via di Frascati 33, 00078 Monte Porzio Catone, Italy

9 INAF - Osservatorio Astronomico di Capodimonte, Via Moiariello 16, 80131 Napoli, Italy

10 Space Telescope Science Institute, 3700 San Martin Drive, Baltimore, MD 21218, USA

11 Cerro Tololo Inter-American Observatory/NSF's NOIRLab, Casilla 603, La Serena, Chile

12 SUPA, School of Science and Engineering, University of Dundee, Nethergate, Dundee DD1 4HN, UK
13 School of Cosmic Physics, Dublin Institute for Advanced Studies, 31 Fitzwilliam Place, Dublin 2, Ireland

14 INAF - Osservatorio Astrofisico di Torino, Via Osservatorio 20, 10025 Pino Torinese, Italy

15 INAF - Osservatorio Astrofisico di Arcetri, Largo Enrico Fermi, 5, 50125 Firenze, Italy

16 Max Planck Institute for Astronomy, Königstuhl 17, 69117 Heidelberg, Germany

17 ELTE Eötvös Loránd University, Institute of Physics, Pázmány Péter sétány 1/A, 1117 Budapest, Hungary

18 Department of Physics, Texas State University, 749 N Comanche Street, San Marcos, TX 78666, USA

19 Univ. Grenoble Alpes, CNRS, IPAG, 38000 Grenoble, France

20 Observatoire de Paris, PSL University, Sorbonne University, CNRS, LERMA, 61 Av. de l'Observatoire, 75014 Paris, France

21 School of Physics, University College Dublin, Belfield, Dublin 4, Ireland

22 Thüringer Landessternwarte, Sternwarte 5, 07778 Tautenburg, Germany

23 Universität Sternwarte München, Ludwig-Maximillian-Universität, Scheinerstrasse 1, 81679 München, Germany

24 Institute for Astrophysical Research, Department of Astronomy, Boston University, 725 Commonwealth Avenue, Boston, MA 02215, USA

25 Purple Mountain Observatory, Chinese Academy of Sciences, 10 Yuanhua Road, Nanjing 210023, PR China

26 Laboratory for Atmospheric and Space Physics, University of Colorado, Boulder, CO 80309, USA

27 Instituto de Astrofísica e Ciências do Espaço, CAUP \& DFA/FCUP, Universidade do Porto, Rua das Estrelas, 4150-762 Porto, Portugal

28 Sterrenkundig Instituut Anton Pannekoek, Science Park 904, 1098 XH Amsterdam, The Netherlands

29 Crimean Astrophysical Observatory, 298409 Nauchny, Crimea

30 MIT Kavli Institute for Astrophysics and Space Research, 77 Massachusetts Avenue, Cambridge, MA 02139, USA

31 California Institute of Technology, 1200 East California Blvd, Pasadena, CA 91125, USA

32 European Space Agency (ESA), European Space Research and Technology Centre (ESTEC), Keplerlaan 1, 2201 AZ Noordwijk, The Netherlands

33 Institute for Astronomy, University of Hawaii at Manoa, Honolulu, USA

34 Dipartimento di Fisica, Universitá degli Studi di Milano, Via Giovanni Celoria 16, 20133 Milano, Italy

35 Instituto de Física y Astronomía, Facultad de Ciencias, Universidad de Valparaíso, Av. Gran Bretaña 1111, 5030 Casilla, Valparaíso, Chile

36 Núcleo Milenio de Formación Planetaria - NPF, Universidad de Valparaíso, Av. Gran Bretaña 1111, Valparaíso, Chile

37 Centro de Astrobiología (CSIC-INTA), Departamento de Astrofísica, ESA-ESAC Campus, PO Box 78, 28691 Villanueva de la Cañada, Madrid, Spain

38 School of Physics and Astronomy, University of Leeds, Leeds LS2 9JT, UK

39 INAF - Osservatorio Astronomico di Padova, Vicolo dell'Osservatorio 5, 35122 Padova, Italy

40 Leiden Observatory, Leiden University, PO Box 9513, 2300 RA Leiden, The Netherlands

41 School of Physics and Astronomy, University of Leicester, Leicester LE1 7RH, UK

42 Hamburger Sternwarte, Gojenbergsweg 112, 21029 Hamburg, Germany

43 Eberhard-Karls Universität Tübingen, Sand 1, 72076 Tübingen, Germany

44 INF - Osservatorio Astronomico di Palermo, Piazza del Parlamento 1, 90134 Palermo, Italy

45 Armagh Observatory and Planetarium, College Hill, Armagh BT61 9DG, UK

46 Stony Brook University, Stony Brook, NY 11794, USA 


\section{Appendix A: Information on the targets from the literature}

Membership and location of the targets within the Orion OB1 association have been thoroughly discussed in Briceño et al. (2019). The subassociation to which each target belongs, as well as spectral types and extinctions from Briceño et al. (2019) are given in Table A.1. The extinctions were determined from the $V-I_{\mathrm{c}}$ color, using standard colors from Kenyon \& Hartmann (1995). Masses and luminosities of the star and accretion luminosities and mass accretion rates have been determined for a subset of the targets. Ingleby et al. (2014) used a combination of low and medium resolution spectrographs (MagE and MIKE on the Magellan observatory) to obtain spectra from $3400 \AA$ to $9000 \AA$ for a sample of CVSO stars, which included CVSO 58, CVSO 90, CVSO 107 and CVSO 109. They determined spectral types, veiling and extinction following procedures similar to Manara et al. (2013b). They fitted the excess flux above the photosphere with multicolumn accretion shock models, and obtained accretion values given in Table A.1. Another subset of the targets, CVSO 104, CVSO 107, and CVSO 109, was included in Maucó et al. (2018), who studied the disks of CVSO stars detected by the PACS instrument on board Herschel. Maucó et al. (2018) used spectral types and extinctions from Briceño et al. (2019) and determined accretion luminosities and mass accretion rates (Table A.1) from the $\mathrm{H} \alpha$ luminosity, estimated from the equivalent width of the line and the $R_{\mathrm{c}}$ magnitude in Briceño et al. (2019). A comparison of Tables 1 and A.1 indicates general agreement between the results of this study and especially those of Ingleby et al. (2014), who used similar methods. The differences may be due to intrinsic variability in CVSO 58, and maybe mismatch of spectral types in CVSO 107.

Young stars located in the field of view of the $\sigma$ Ori cluster belong to one of the two stellar groups kinematically separated in radial velocities (Jeffries et al. 2006). One group
( $\mathrm{RV} \sim 27-37 \mathrm{~km} \mathrm{~s}^{-1}$ ) is associated with the $\sigma$ Ori cluster, and the other group ( $\mathrm{RV} \sim 20-27 \mathrm{~km} \mathrm{~s}^{-1}$ ) includes stars that belong to a sparse stellar population located in front of the $\sigma$ Orionis cluster (e.g., Pérez-Blanco et al. 2018). Based on radial velocity measurements, the kinematic membership of the stars SO 518 and SO 583 was confirmed by Hernández et al. (2014). The radial velocity of $33.4 \mathrm{~km} \mathrm{~s}^{-1}$ obtained from the Sloan Digital Sky Survey Sky Archive Server (SDDS-SAS ${ }^{10}$ ), suggests that the star SO1183 is also a bona fide member of the $\sigma$ Ori cluster. This radial velocity was measured from high-resolution $\mathrm{H}$-band spectra using the APOGEE Stellar Parameter and Chemical Abundance Pipeline (ASPCAP; García Pérez et al. 2016). The radial velocities obtained in this work (Tables 2-4) are in agreement with these previous estimates.

The youth of SO 518, SO 583, and SO 1153 is also confirmed by lithium in absorption, infrared excesses, and strong $\mathrm{H} \alpha$ in emission (Hernández et al. 2014; Maucó et al. 2016). In addition, they have been detected by Spitzer (Hernández et al. 2007a), Herschel (Maucó et al. 2016), and ALMA (Ansdell et al. 2017). Stellar and accretion properties from the literature are shown in Table A.2. For stars SO 518 and SO 583, spectral types from Hernández et al. (2014) agree within the uncertainties with the values used in this work; however, for SO 1153 there is a difference of 1.5 sub-types. The spectral energy distribution (SED) of this star suggest that it is a Class I object (Hernández et al. 2007b), with variability amplitude of $0.53 \mathrm{mag}$ (Cody \& Hillenbrand 2010) that could be due to disk obscuration. These conditions could make it difficult to obtain more precise stellar parameters. Extinction values and stellar parameters were estimated by Maucó et al. (2016), as well as mass accretion rates, from the luminosity of the $\mathrm{H} \alpha$ line. Significant differences exist between estimates of $A_{V}$, stellar luminosities, and accretion rates from the literature, Table A.2, and those in this work, Table 1 . They are mostly due to including veiling estimates in the determinations, variability, as well as to the different methods for obtaining the extinction.

Table A.1. Stellar and accretion properties from the literature for the targets in the Orion OB1a and OB1b Associations.

\begin{tabular}{|c|c|c|c|c|c|c|c|c|c|c|c|}
\hline Target & 2MASS & Dist. & $\mathrm{SpT}^{(a)}$ & $\mathrm{SpT}^{(b)}$ & $A_{V}{ }^{(a)}$ & $A_{V}{ }^{(b)}$ & $M_{\star}^{(b)}$ & $L_{\star}^{(b)}$ & $\log \left(\dot{M}_{\mathrm{acc}}\right)^{(b)}$ & $\log \left(\dot{M}_{\mathrm{acc}}\right)^{(c)}$ & Loc \\
\hline CVSO-17 & $\mathrm{J} 05230470+0137148$ & 330 & M3 & - & 0.0 & - & - & - & - & - & $1 \mathrm{a}$ \\
\hline CVSO-36 & $\mathrm{J} 05255035+0149370$ & 330 & M3 & - & 0.0 & - & - & - & - & - & $1 \mathrm{a}$ \\
\hline CVSO-58 & J05292326-0125153 & 440 & K7 & K7.5 & 1.2 & 0.8 & 0.8 & 0.8 & -7.80 & - & $1 b$ \\
\hline CVSO-90 & J05312062-0049197 & 440 & K7 & M0.5 & 0.0 & 0.0 & 0.4 & 0.3 & -8.00 & - & $1 b$ \\
\hline CVSO-104 & J05320638-0111000 & 440 & K7 & - & 0.3 & - & - & - & - & -8.25 & $1 b$ \\
\hline CVSO-107 & J05322578-0036533 & 440 & K7 & K7.5 & 1.4 & 0.7 & 0.8 & 0.9 & -8.60 & -8.53 & $1 b$ \\
\hline CVSO-109 & J05323265-0113461 & 440 & M0 & K7.5 & 0.4 & 0.8 & 0.6 & 1.5 & -7.52 & -8.17 & $1 b$ \\
\hline CVSO-146 & J05354600-0057522 & 440 & K6 & - & 0.2 & - & - & - & - & - & $1 b$ \\
\hline CVSO-165 & J05390257-0120323 & 440 & K6 & - & 0.6 & - & - & - & - & - & $1 b$ \\
\hline CVSO-176 & J05402414-0031213 & 440 & M3 & - & 0.8 & - & - & - & - & - & $1 b$ \\
\hline
\end{tabular}

Notes. ${ }^{(a)}$ Briceño et al. (2019), ${ }^{(b)}$ Ingleby et al. (2014), ${ }^{\left({ }^{(c)}\right.}$ Maucó et al. (2018). Distances are reported in parsec.

\footnotetext{
$\overline{10}$ https://dr16.sdss.org/infrared/spectrum/search
} 
Table A.2. Stellar and accretion properties from the literature for the targets in the $\sigma$ Ori cluster.

\begin{tabular}{lccccccccc}
\hline \hline Target & 2MASS & $\mathrm{RV}^{(a)}$ & $\mathrm{RV}^{(b)}$ & $\mathrm{SpT}^{(a)}$ & $A_{V}{ }^{(a)}$ & $M_{\star}{ }_{\star}^{(c)}$ & $L_{\star}{ }^{(c)}$ & $\operatorname{Disk}_{\text {type }}^{(c)}$ & $\log \left(\dot{M}_{\mathrm{acc}}\right)^{(c)}$ \\
\hline SO 518 & J05382725-0245096 & $28.8 \pm 0.6$ & 30.3 & $\mathrm{~K} 6.0 \pm 1.0$ & 0.0 & 0.75 & 0.44 & $\mathrm{II}$ & -8.54 \\
SO 583 & J05383368-0244141 & $29.5 \pm 0.5$ & 31.5 & $\mathrm{~K} 4.5 \pm 1.5$ & 0.0 & 1.09 & 2.57 & II & -8.13 \\
SO 1153 & J05393982-0231217 & $\ldots$ & 33.4 & $\mathrm{~K} 5.5 \pm 1.0$ & 0.15 & 0.88 & 0.52 & $\mathrm{I}$ & -8.38 \\
\hline
\end{tabular}

Notes. ${ }^{(a)}$ Hernández et al. (2014), ${ }^{(b)}$ SDSS-SAS, ${ }^{(c)}$ Maucó et al. (2018). Radial Velocities are reported in $\mathrm{km} \mathrm{s}^{-1}$, stellar masses are reported in solar masses, and ages are reported in Myr.

\section{Appendix B: Observation log}

The weather conditions at the time of the observations were always extremely good in this campaign, with clear (CLR) or photometric (PHO) sky conditions. There was no major issue observed in the data, apart from the saturation of the $\mathrm{H} \alpha$ line in the third epoch of the UVES observations of CVSO 109. Typically, image quality - seeing corrected for airmass of the observations - was about $1^{\prime \prime}$, as reported in Table B.1-B.3.

Figures B.1 and B.2 show the time at which the targets have been observed, together with highlights of the time of start and end of the HST observations.

Table B.1. X-shooter observations log.

\begin{tabular}{|c|c|c|c|c|c|c|}
\hline \multirow[t]{2}{*}{ Name } & \multirow[t]{2}{*}{ Date of observation [UT] } & \multirow{2}{*}{$\begin{array}{c}\text { Exp. time } \\
{[N \exp \times(s)]}\end{array}$} & \multicolumn{3}{|c|}{ Slit width ["] } & \multirow{2}{*}{$\begin{array}{l}\text { I.Q. } \\
{\left[{ }^{\prime \prime}\right]}\end{array}$} \\
\hline & & & UVB & VIS & NIR & \\
\hline CVSO 17 & 2020-12-05Т02:39:59.716 & $2 \times 900$ & 1.0 & 0.4 & 0.4 & 1.06 \\
\hline CVSO 36 & 2020-12-03Т02:48:38.197 & $2 \times 900$ & 1.0 & 0.4 & 0.4 & 0.95 \\
\hline CVSO 58 & 2020-12-02Т06:06:48.094 & $2 \times 460$ & 1.0 & 0.4 & 0.4 & 0.91 \\
\hline CVSO 90 & 2020-12-15T01:19:38.340 & $2 \times 460$ & 1.0 & 0.4 & 0.4 & 1.18 \\
\hline CVSO 104 & 2020-11-27Т03:06:13.092 & $2 \times 370$ & 1.0 & 0.4 & 0.4 & 1.15 \\
\hline CVSO 107 & 2020-12-04T01:54:01.513 & $2 \times 460$ & 1.0 & 0.4 & 0.4 & 0.99 \\
\hline CVSO 109 & 2020-11-28Т03:36:03.569 & $2 \times 470$ & 1.0 & 0.4 & 0.4 & 0.89 \\
\hline CVSO 146 & 2020-12-09T01:50:50.818 & $2 \times 390$ & 1.0 & 0.4 & 0.4 & 1.05 \\
\hline CVSO 165 & 2020-12-14T01:50:09.831 & $2 \times 390$ & 1.0 & 0.4 & 0.4 & 1.16 \\
\hline CVSO 176 & 2020-12-02Т04:09:15.119 & $2 \times 900$ & 1.0 & 0.4 & 0.4 & 1.11 \\
\hline SO 518 & 2020-12-02Т03:21:40.090 & $2 \times 300$ & 0.5 & 0.4 & 0.4 & 1.21 \\
\hline SO 583 & 2020-12-02T03:44:41.430 & $2 \times 300$ & 0.5 & 0.4 & 0.4 & 1.11 \\
\hline SO 1153 & 2020-12-07Т04:47:11.315 & $2 \times 490$ & 0.5 & 0.4 & 0.4 & 0.79 \\
\hline
\end{tabular}

Notes. Typical resolutions are: in the UVB arm $R \sim 9700$ and $R \sim 5400$ for the $0.5^{\prime \prime}$ and $1.0^{\prime \prime}$ wide slit, respectively; in the VIS arm $R \sim 18400$ and $R \sim 8900$ for the $0.4^{\prime \prime}$ and $0.9^{\prime \prime}$ wide slit, respectively; in the NIR arm $R \sim 11600$ and $R \sim 5600$ for the $0.4^{\prime \prime}$ and $0.9^{\prime \prime}$ wide slit, respectively. Exposure times are reported in the UVB arm, similar to the NIR arms within $\sim 5-10 \mathrm{~s}$, whereas the corresponding VIS exposures are $\sim 60-90 \mathrm{~s}$ shorter. I.Q. is the airmass corrected seeing. 
Table B.2. UVES observations log.

\begin{tabular}{llcc}
\hline \hline Name & Date of observation [UT] & $\begin{array}{c}\text { Exp. time } \\
{[\text { Nexp } \times(\mathrm{s})]}\end{array}$ & $\begin{array}{c}\text { I.Q. } \\
{\left[{ }^{\prime \prime}\right]}\end{array}$ \\
\hline CVSO 17 & $2020-12-04 T 04: 02: 13.793$ & $2 \times 1800$ & 0.81 \\
CVSO 17 & $2020-12-05 T 02: 16: 35.606$ & $2 \times 1800$ & 0.97 \\
CVSO 17 & $2020-12-06 T 01: 53: 46.058$ & $2 \times 1800$ & 1.15 \\
\hline CVSO 36 & $2020-12-02 T 03: 59: 25.264$ & $2 \times 1800$ & 1.04 \\
CVSO 36 & $2020-12-03 T 02: 32: 58.168$ & $2 \times 1800$ & 0.88 \\
CVSO 36 & $2020-12-04 T 02: 56: 47.849$ & $2 \times 1800$ & 0.99 \\
\hline CVSO 58 & $2020-11-30 T 02: 23: 51.801$ & $2 \times 1300$ & 1.24 \\
CVSO 58 & $2020-12-01 T 02: 22: 57.299$ & $2 \times 1300$ & 1.04 \\
CVSO 58 & $2020-12-02 T 02: 09: 45.709$ & $2 \times 1300$ & 1.23 \\
\hline CVSO 104 & $2020-11-25 T 03: 09: 25.349$ & $2 \times 1100$ & 1.12 \\
CVSO 104 & $2020-11-26 T 02: 48: 15.196$ & $2 \times 1100$ & 1.13 \\
CVSO 104 & $2020-11-27 T 02: 22: 14.815$ & $2 \times 1100$ & 1.10 \\
\hline CVSO 107 & $2020-12-03 T 03: 38: 39.578$ & $2 \times 1250$ & 1.31 \\
CVSO 107 & $2020-12-04 T 02: 10: 09.193$ & $2 \times 1250$ & 1.00 \\
CVSO 107 & $2020-12-05 T 03: 22: 18.754$ & $2 \times 1250$ & 1.23 \\
\hline CVSO 109 & $2020-11-26 T 03: 29: 45.199$ & $2 \times 1050$ & 0.91 \\
CVSO 109 & $2020-11-27 T 03: 02: 45.220$ & $2 \times 1050$ & 0.98 \\
CVSO 109 & $2020-11-28 T 03: 59: 35.437$ & $2 \times 1050$ & 0.88 \\
\hline CVSO 176 & $2020-11-28 T 02: 52: 44.394$ & $2 \times 1800$ & 0.98 \\
CVSO 176 & $2020-11-29 T 02: 35: 28.950$ & $2 \times 1800$ & 0.88 \\
CVSO 176 & $2020-11-30 T 03: 30: 00.408$ & $2 \times 1800$ & 1.15 \\
\hline SO 518 & $2020-11-29 T 03: 40: 36.060$ & $2 \times 1100$ & 0.86 \\
SO 518 & $2020-11-30 T 04: 35: 15.871$ & $2 \times 1100$ & 1.07 \\
SO 518 & $2020-12-01 T 03: 28: 58.125$ & $2 \times 1100$ & 0.91 \\
\hline SO 583 & $2020-11-29 T 04: 22: 04.942$ & $2 \times 420$ & 0.69 \\
SO 583 & $2020-11-30 T 03: 11: 49.882$ & $2 \times 420$ & 1.06 \\
SO 583 & $2020-12-01 T 03: 10: 36.338$ & $2 \times 420$ & 0.84 \\
\hline
\end{tabular}

Notes. I.Q. is the airmass corrected seeing.

Table B.3. ESPRESSO observations log.

\begin{tabular}{lccc}
\hline \hline Name & Date of observation [UT] & $\begin{array}{c}\text { Exp. time } \\
{[\text { Nexp } \times(\mathrm{s})]}\end{array}$ & $\begin{array}{c}\text { I.Q. } \\
{\left[{ }^{\prime \prime}\right]}\end{array}$ \\
\hline CVSO 146 & $2020-12-09 T 02: 42: 01.462$ & $2 \times 1205$ & 1.26 \\
CVSO 146 & $2020-12-10 T 02: 09: 50.465$ & $2 \times 1205$ & 1.17 \\
CVSO 146 & $2020-12-11$ T01:55:43.819 & $2 \times 1205$ & 1.21 \\
\hline CVSO 165 & $2020-12-13 T 01: 35: 50.344$ & $2 \times 1080$ & \\
CVSO 165 & $2020-12-14 T 02: 18: 48.659$ & $2 \times 1080$ & \\
CVSO 165 & $2020-12-15 T 02: 30: 53.897$ & $2 \times 1080$ & \\
\hline CVSO 90 & $2020-12-15 T 03: 11: 36.839$ & $2 \times 1670$ & \\
\hline SO 1153 & $2020-12-08 T 07: 38: 54.786$ & $2 \times 1330$ & \\
SO 1153 & $2020-12-09 T 01: 52: 07.779$ & $2 \times 1330$ & 0.98 \\
SO 1153 & $2020-12-10 T 02: 55: 38.503$ & $2 \times 1330$ & 0.89 \\
\hline
\end{tabular}

Notes. I.Q. is the airmass corrected seeing. 

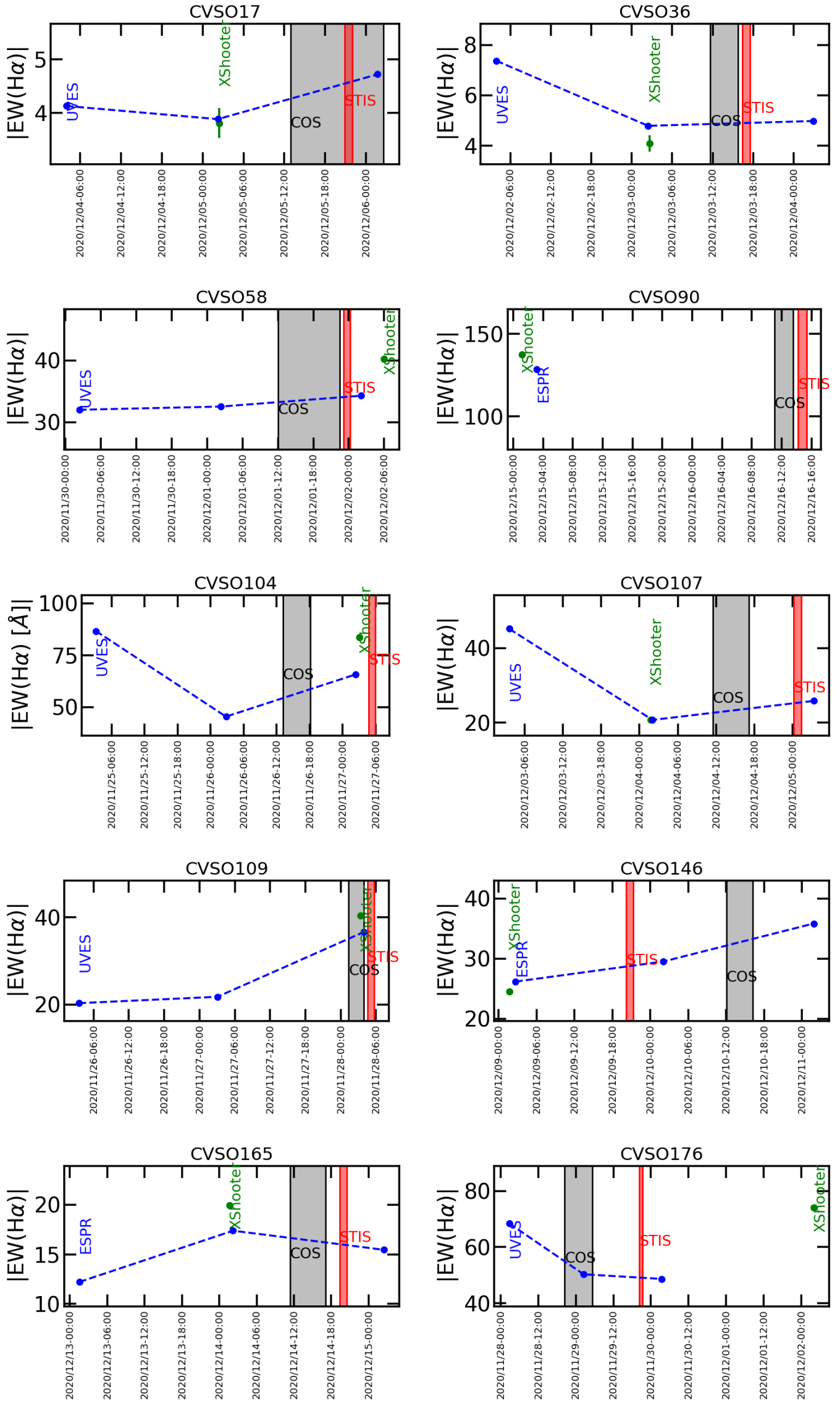

Fig. B.1. Variability of the $\mathrm{H} \alpha$ equivalent width for the Orion OB1 targets as measured by EPRESSO or UVES (blue) and X-shooter (green), together with the timining of the COS (grey) and STIS (red) HST observations. 

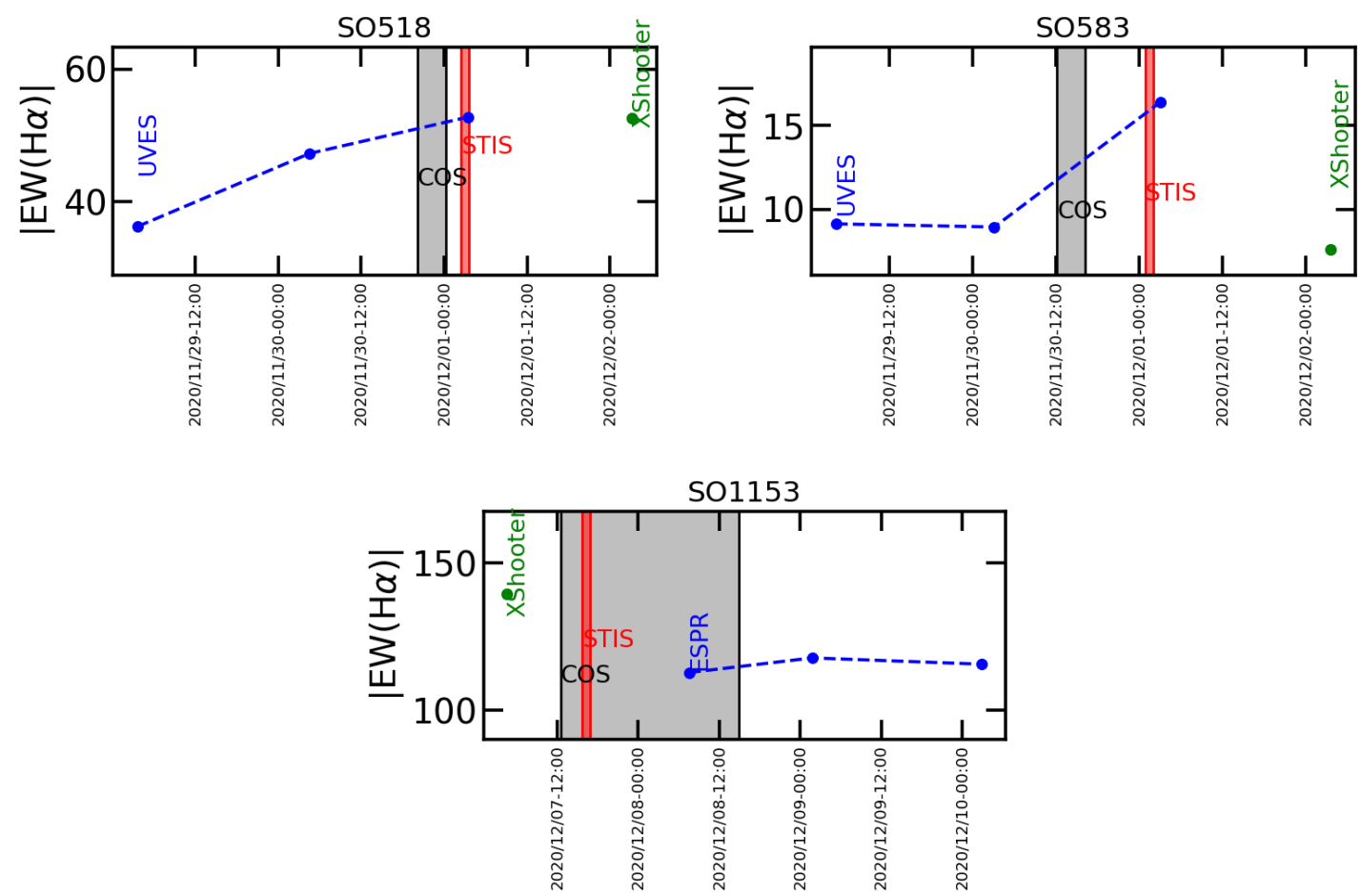

Fig. B.2. Variability of the $\mathrm{H} \alpha$ equivalent width for the $\sigma$-Orionis targets as measured by EPRESSO or UVES (blue) and X-shooter (green), together with the timining of the COS (grey) and STIS (red) HST observations. 


\section{Appendix C: Templates used for the ROTFIT analysis}

As described in Sect. 2.5.2, a number of main sequence stars observed with the HARPS spectrograph are used to fit the high- resolution spectra of our targets. Table C.1 reports the information about these targets, collected from Grandjean et al. (2020).

Table C.1. HARPS templates.

\begin{tabular}{|c|c|c|c|c|c|c|c|}
\hline \multirow[t]{2}{*}{ Name } & \multirow[t]{2}{*}{$\mathrm{SpT}$} & \multirow{2}{*}{$\begin{array}{c}T_{\mathrm{eff}} \\
{[\mathrm{K}]}\end{array}$} & $\log g$ & {$[\mathrm{Fe} / \mathrm{H}]$} & RV & $v \sin i$ & \multirow[t]{2}{*}{ References } \\
\hline & & & \multicolumn{2}{|c|}{ [dex] } & \multicolumn{2}{|c|}{$\left[\mathrm{km} \mathrm{s}^{-1}\right]$} & \\
\hline HD4628 & $\mathrm{K} 2 \mathrm{~V}$ & 5035 & 4.60 & -0.27 & -10.165 & 0.7 & Lu2018, Br2016 \\
\hline HD16160 & K3V & 4858 & 4.89 & -0.18 & 25.874 & 0.9 & Ri2017, Mi2012 \\
\hline HD32147 & $\mathrm{K} 3 \mathrm{~V}$ & 4780 & 4.56 & 0.37 & 21.624 & 1.7 & Lu2018, Va2005 \\
\hline HD24916 & $\mathrm{K} 4 \mathrm{~V}$ & 4696 & 4.50 & -0.12 & 3.64 & 3.5 & Mo2018, G12005 \\
\hline HD35171 & $\mathrm{K} 4 \mathrm{~V}$ & 4576 & 4.70 & 0.02 & 38.267 & 3.4 & Mi2012, Mi2012 \\
\hline HD154363 & $\mathrm{K} 5 \mathrm{~V}$ & 4373 & 4.66 & -0.32 & 34.146 & 2.5 & $\mathrm{Lu} 2017$ \\
\hline HD218511 & $\mathrm{K} 5.5 \mathrm{~V}$ & 4361 & 4.61 & -0.09 & 4.654 & 1.0 & Lu2018 \\
\hline HD200779 & K6V & 4406 & 4.62 & 0.08 & -66.85 & 2.0 & Lu2018, Hou2016 \\
\hline HD35650 & K6V & 4269 & 4.65 & 0.06 & 32.264 & 4.0 & Lu2018, To2006 \\
\hline HIP116384 & K7V & 4180 & 4.70 & -0.10 & -10.3 & $<2.0$ & Zb1998, Нoj2019 \\
\hline HIP17157 & $\mathrm{K} 7 \mathrm{~V}$ & 4128 & 4.28 & 0.08 & 23.90 & 5.0 & Mc2017, Gr2020 \\
\hline BD-08 2582 & K8V & 4044 & 4.66 & 0.13 & 37.035 & $<2.0$ & Li2017, Hoj2019 \\
\hline GJ488 & MOV & 3989 & 4.66 & 0.24 & 5.041 & 2.7 & Ye2017, Hoj2019 \\
\hline HD209290 & $\mathrm{M} 0.5 \mathrm{~V}$ & 3914 & 4.69 & 0.05 & 18.363 & $<2.0$ & Pa2018, Je2018 \\
\hline HD42581 & M1V & 3822 & 4.71 & 0.06 & 4.734 & 2.6 & Pa2018, Hou 2016 \\
\hline GJ514 & M1.0V & 3727 & 4.78 & 0.07 & 14.606 & 2.1 & Li2017, Hou2016 \\
\hline HD165222 & M1.5V & 3664 & 4.87 & -0.21 & 33.045 & 1.6 & Pa2018, Hou2016 \\
\hline HD119850 & M1.5V & 3677 & 4.79 & -0.04 & 15.570 & 1.0 & Pa2018, Hou 2016 \\
\hline HD217987 & $\mathrm{M} 2 \mathrm{~V}$ & 3680 & 4.88 & -0.22 & 8.809 & 1.0 & Wo2005, To2006 \\
\hline HIP51317 & $\mathrm{M} 2 \mathrm{~V}$ & 3575 & 4.89 & -0.09 & 8.35 & 0.1 & $\mathrm{~Pa} 2018, \mathrm{Ga} 2016$ \\
\hline GJ2066 & $\mathrm{M} 2 \mathrm{~V}$ & 3589 & 4.86 & -0.06 & 62.215 & $<2.0$ & Pa2018, Je2018 \\
\hline GJ250B & $\mathrm{M} 2.5 \mathrm{~V}$ & 3569 & 4.84 & 0.01 & -5.40 & $<2.5$ & Ro2012, Re2012 \\
\hline GJ752A & M3V & 3557 & 4.86 & 0.00 & 35.737 & 1.2 & Pa2018, Hou2016 \\
\hline GJ581 & $\mathrm{M} 3.5 \mathrm{~V}$ & 3441 & 4.98 & -0.08 & -9.662 & $<2.0$ & Sc2019, Re2018 \\
\hline GJ105B & M4V & 3392 & 4.81 & 0.05 & 26.815 & 2.4 & Pa2019, Hou2016 \\
\hline GJ699 & $\mathrm{M} 4.5 \mathrm{~V}$ & 3278 & 5.10 & -0.12 & -110.506 & 2.0 & Pa2018, Hou2016 \\
\hline GJ447 & $\mathrm{M} 4.5 \mathrm{~V}$ & 3251 & 5.10 & -0.04 & -31.087 & 2.2 & Pa2018, Hou 2016 \\
\hline GJ83.1 & M5.0V & 3185 & 5.15 & -0.18 & -28.832 & $<2.0$ & Pa2018, Je2018 \\
\hline GJ1002 & M5.5V & 3038 & 5.04 & -0.10 & -40.058 & $<2.0$ & Pa2019, Je2018 \\
\hline GJ551 & M5.5V & 2927 & 5.02 & -0.07 & -20.471 & 2.7 & Pa2016, То2016 \\
\hline Wolf359 & M6.5V & 2900 & 5.40 & 0.18 & 19.413 & $<2.0$ & Ra2018, Je2018 \\
\hline
\end{tabular}

Notes. Br2016= Brewer et al. (2016); Ga2016=Gagné et al. (2016); Gl2005=Glebocki \& Gnacinski (2005); Gr2020=Grandjean et al. (2020); Hoj2019 = Hojjatpanah et al. (2019); Hou2016 = Houdebine et al. (2016); Je2018 = Jeffers et al. (2018); Li2017= Lindgren \& Heiter (2017); Lu2017 = Luck (2017); Lu2018 = Luck (2018); Mc2017 = McDonald et al. (2017); Mi2012= Mishenina et al. (2012); Mo2018= Montes et al. (2018); Pa2016 = Passegger et al. (2016); Pa2018 = Passegger et al. (2018); Pa2019= Passegger et al. (2019); Ra2018=Rajpurohit et al. (2018); Re2012 = Reiners et al. (2012); Re2018 = Reiners et al. (2018); Ri2017=Rich et al. (2017); Ro2012=Rojas-Ayala et al. (2012); Sc2019 = Schweitzer et al. (2019); To2006 = Torres et al. (2006); Va2005 = Valenti \& Fischer (2005); Wo2005 = Woolf \& Wallerstein (2005); Ye2017 = Yee et al. (2017); Zb1998 = Zboril \& Byrne (1998). 


\section{Appendix D: Light curves from multi-band photometry}

We show in Fig. D.1 the plots with the light curves from OACT, $\mathrm{CrAO}$ and AAVSO from mid-November to mid-December. The epochs of HST and VLT observations are also marked as vertical lines in the boxes with $I_{\mathrm{C}}$ light curves. The synthetic photom- etry made on the flux-calibrated X-shooter spectra is overlaid with purple asterisks. For SO 583 and SO 518, we display in the upper panels the index $\mathrm{H} \alpha_{18}-\mathrm{H} \alpha_{9}$, which measures the intensity of the $\mathrm{H} \alpha$ emission, from OACT narrow-band photometry and from UVES (green asterisks) and X-shooter (purple asterisks) spectra.
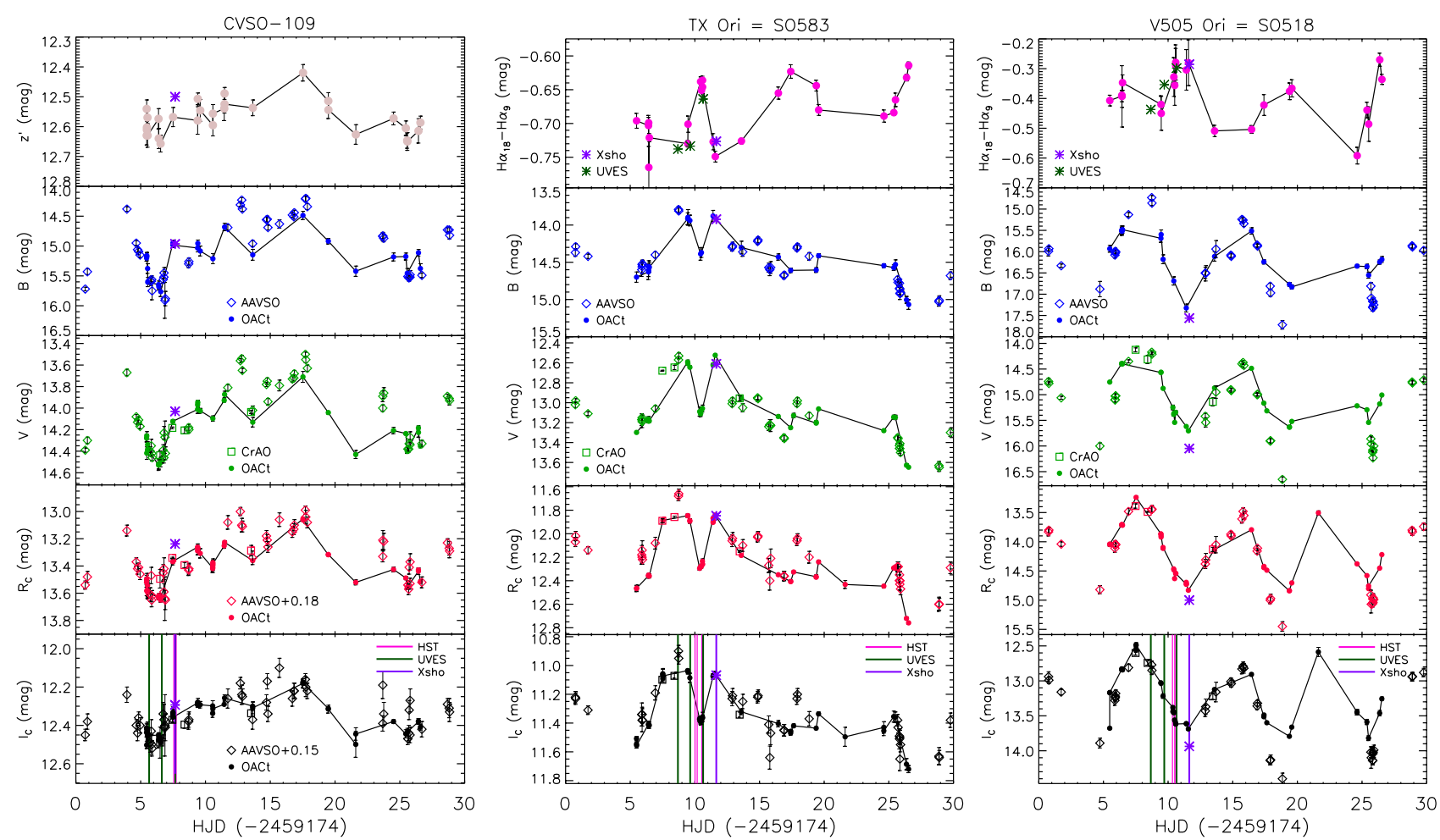

Fig. D.1. Light curves for our targets around the time of our VLT observations. Synthetic photometry on the X-shooter spectra is shown with purple asterisks. 


\section{Appendix E: Plots of the Balmer jump fits}

Here, we show the best fit of the X-shooter spectra of our targets obtained with the method by Manara et al. (2013a) described in
Sect. 2.5.1. The Balmer jump region of the spectra is shown in Figs. E.1 and E.2, while the whole best fit spectra are shown in Figs. E. 3 and E.4.
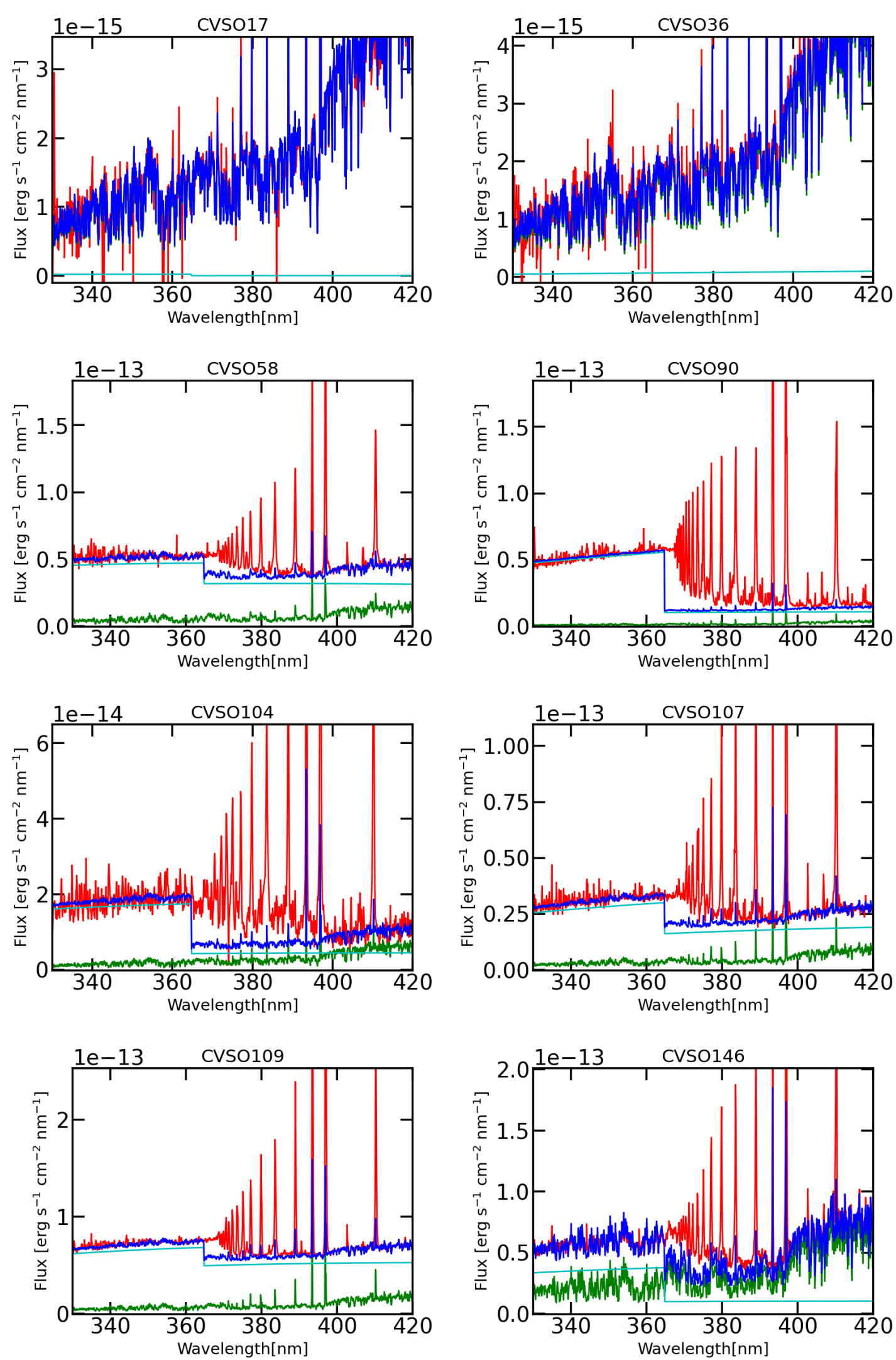

Fig. E.1. Best fit for the Balmer continuum region for the targets in the OB1 association. 

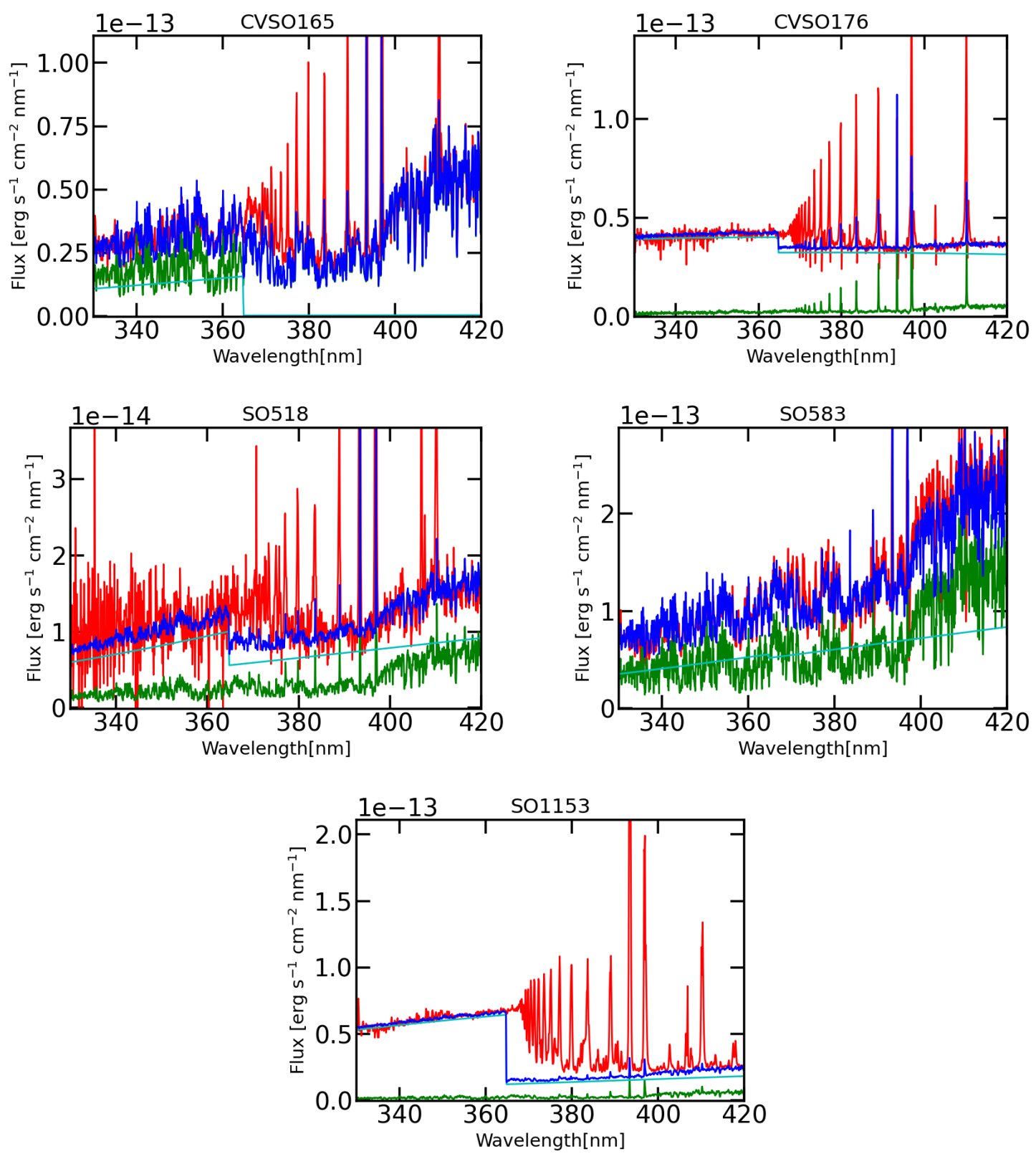

Fig. E.2. Best fit for the Balmer continuum region for the targets in the $\mathrm{OB} 1$ association and $\sigma$-Orionis cluster. 

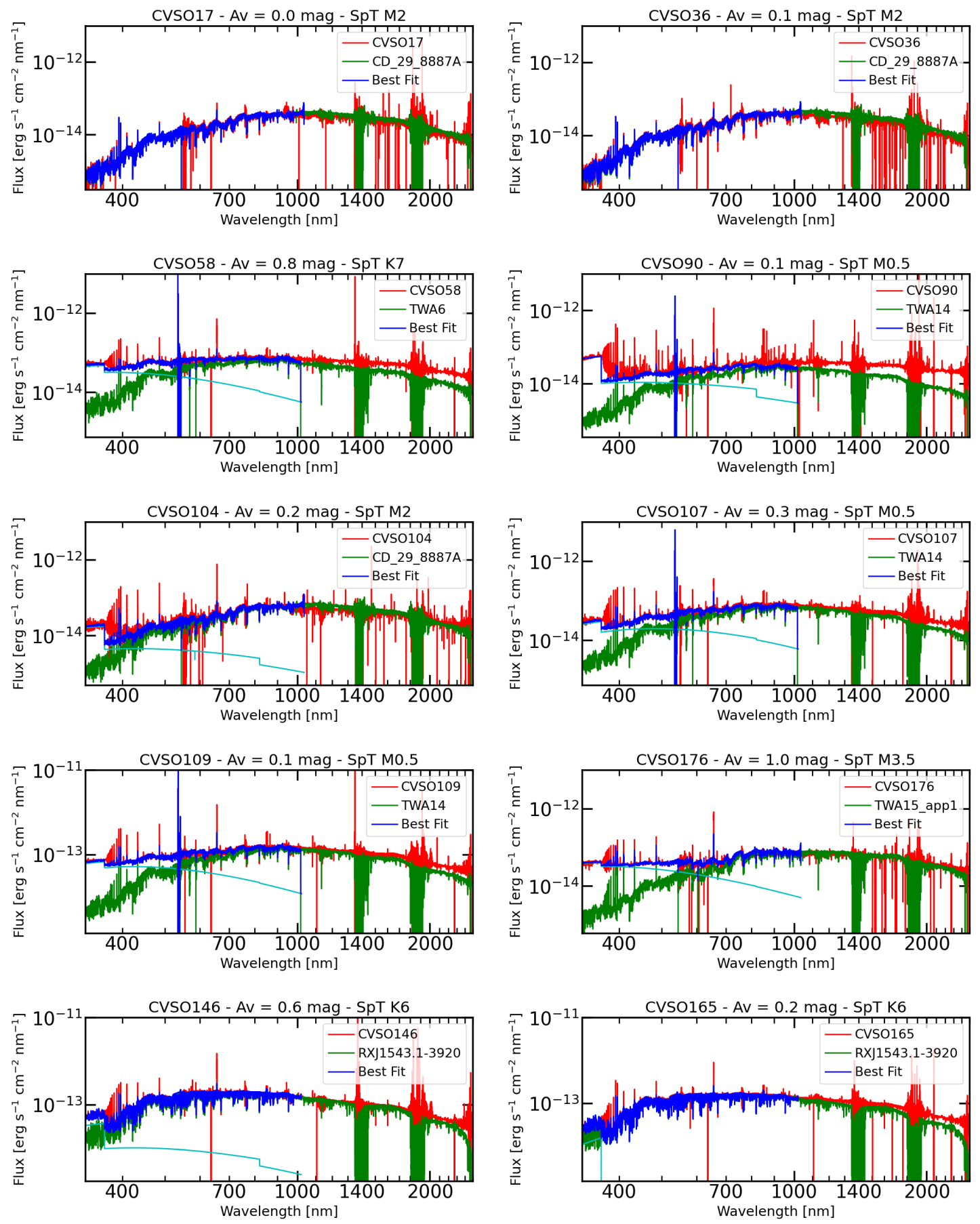

Fig. E.3. X-shooter spectra of Orion OB1 targets (red) with the best fit, composed of a photospheric template (green) and a slab model for the accretion spectrum (cyan). 
C. F. Manara et al.: PENELLOPE. I. Survey presentation and Orion
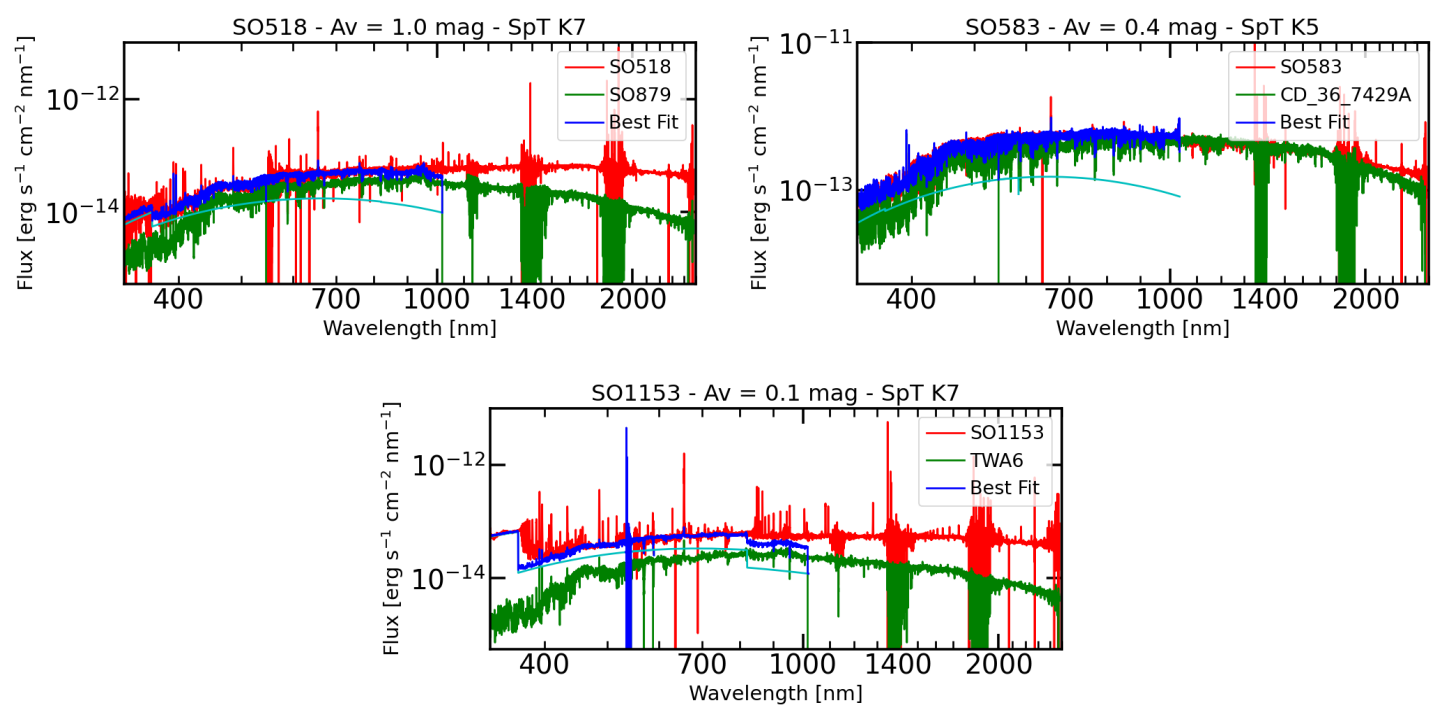

Fig. E.4. X-shooter spectra of $\sigma$-Orionis targets (red) with the best fit, composed of a photospheric template (green) and a slab model for the accretion spectrum (cyan). 


\section{Appendix F: Comparison between HST and VLT/X-shooter spectra}

The comparison between the observed VLT/X-shooter spectra and the HST/STIS ones is shown in Fig. F.1.
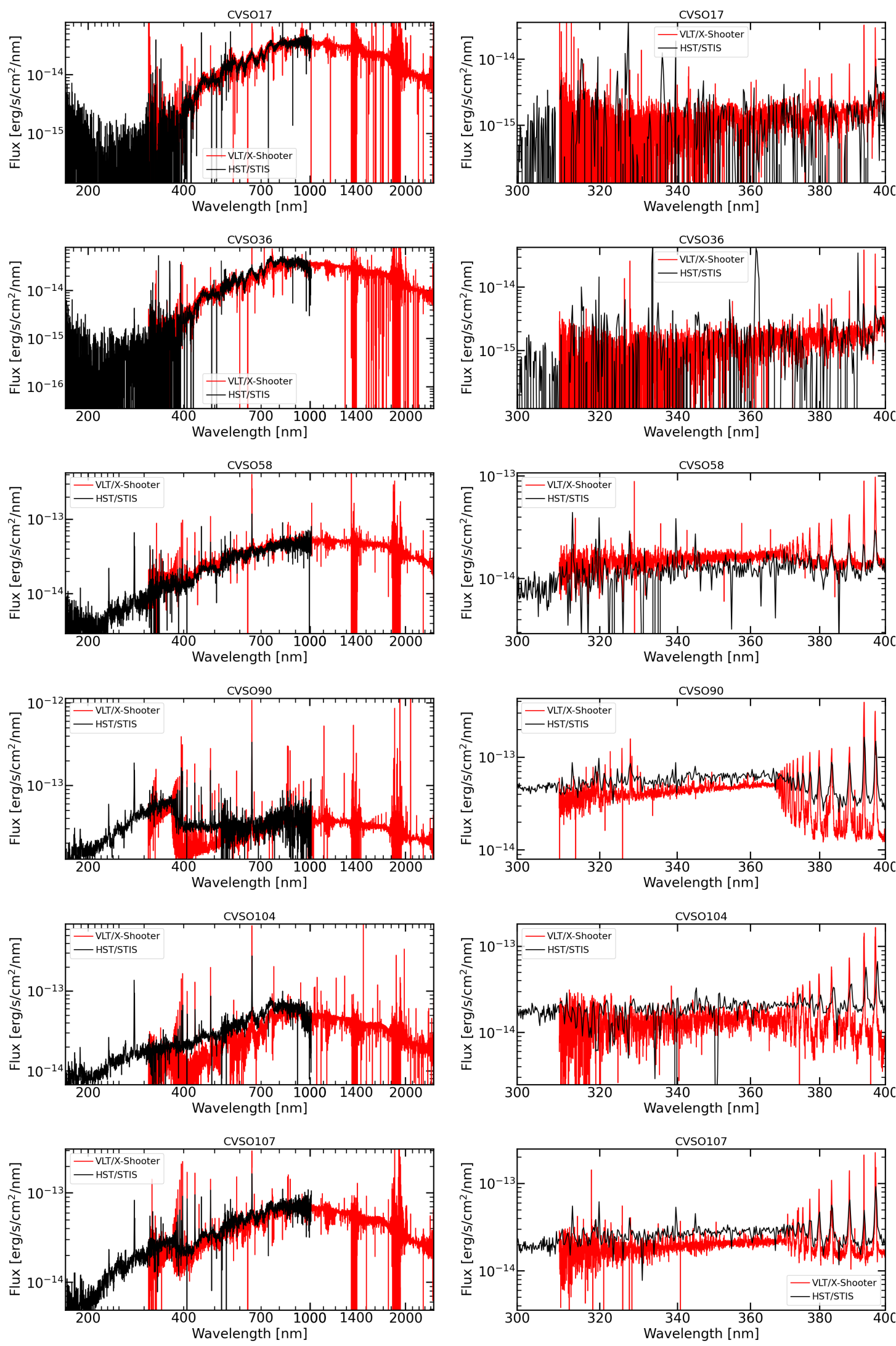

Fig. F.1. Comparison between the observed VLT/X-shooter (red) and HST/STIS (black) spectra. 
C. F. Manara et al.: PENELLOPE. I. Survey presentation and Orion
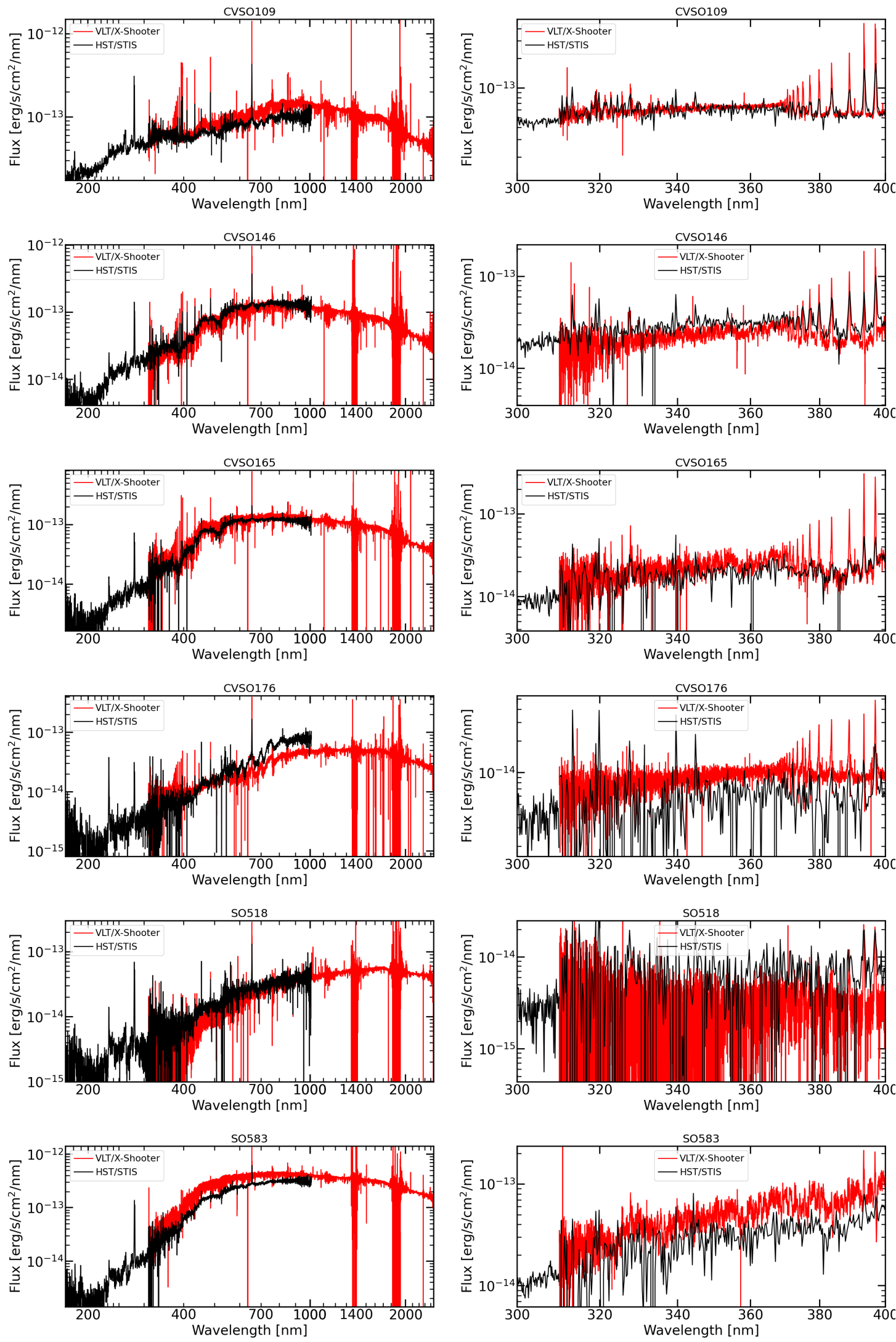

Fig. F.1. continued. 
Appendix G: Line profiles for the observed Orion targets

The profile of the $\mathrm{H} \alpha, \mathrm{H} \beta, \mathrm{HeI} 5876 \AA$, and [OI] $\lambda 6300 \AA$ lines are shown in Figs. G.1-G.13. The X-shooter spectra, shown in blue in all figures, have lower resolution $(R \sim 10000-20000)$ than the UVES $(R \sim 70000)$ and ESPRESSO $(R \sim 140000)$ ones.
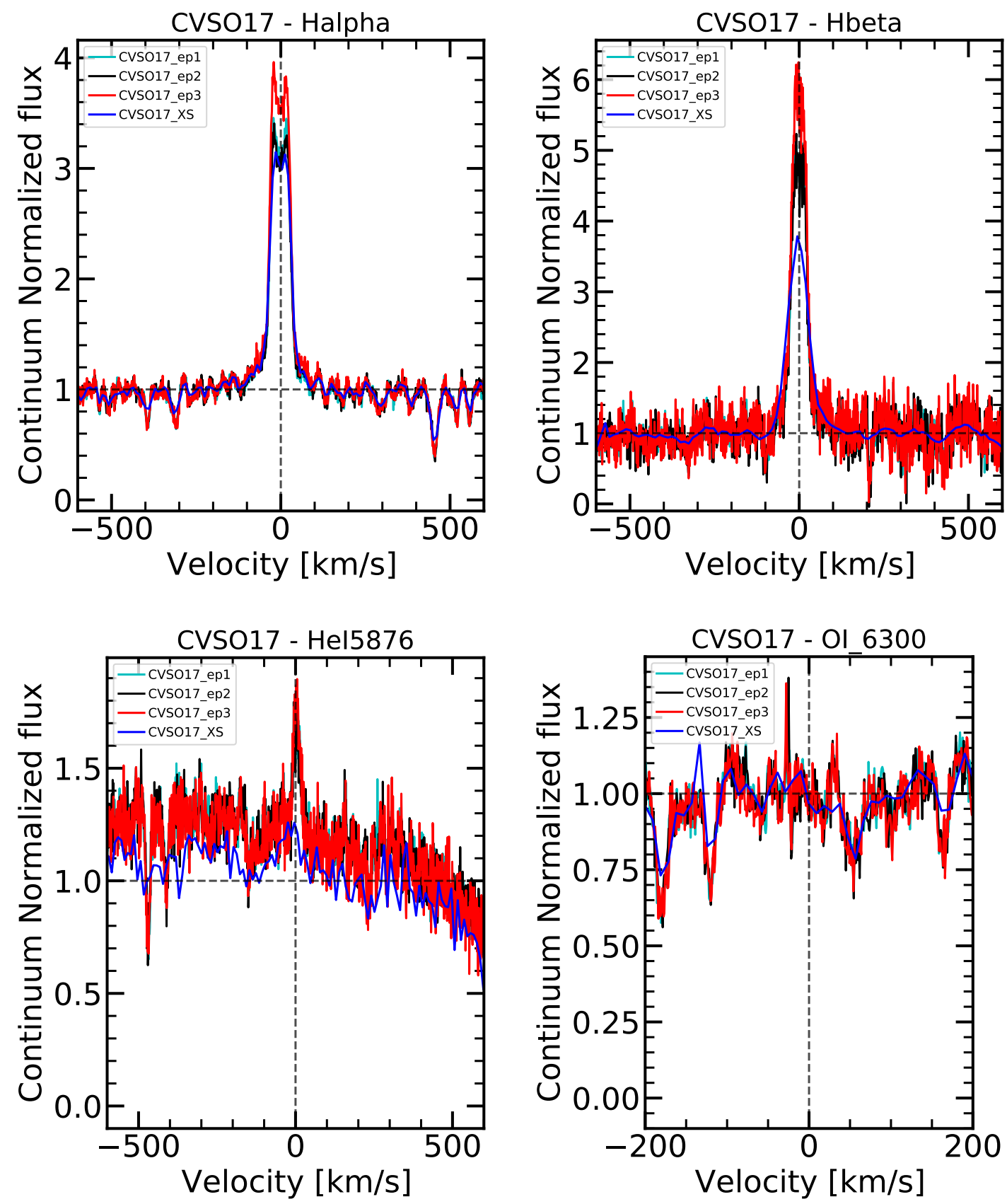

Fig. G.1. Emission lines of the target CVSO 17 observed with UVES and X-shooter. 

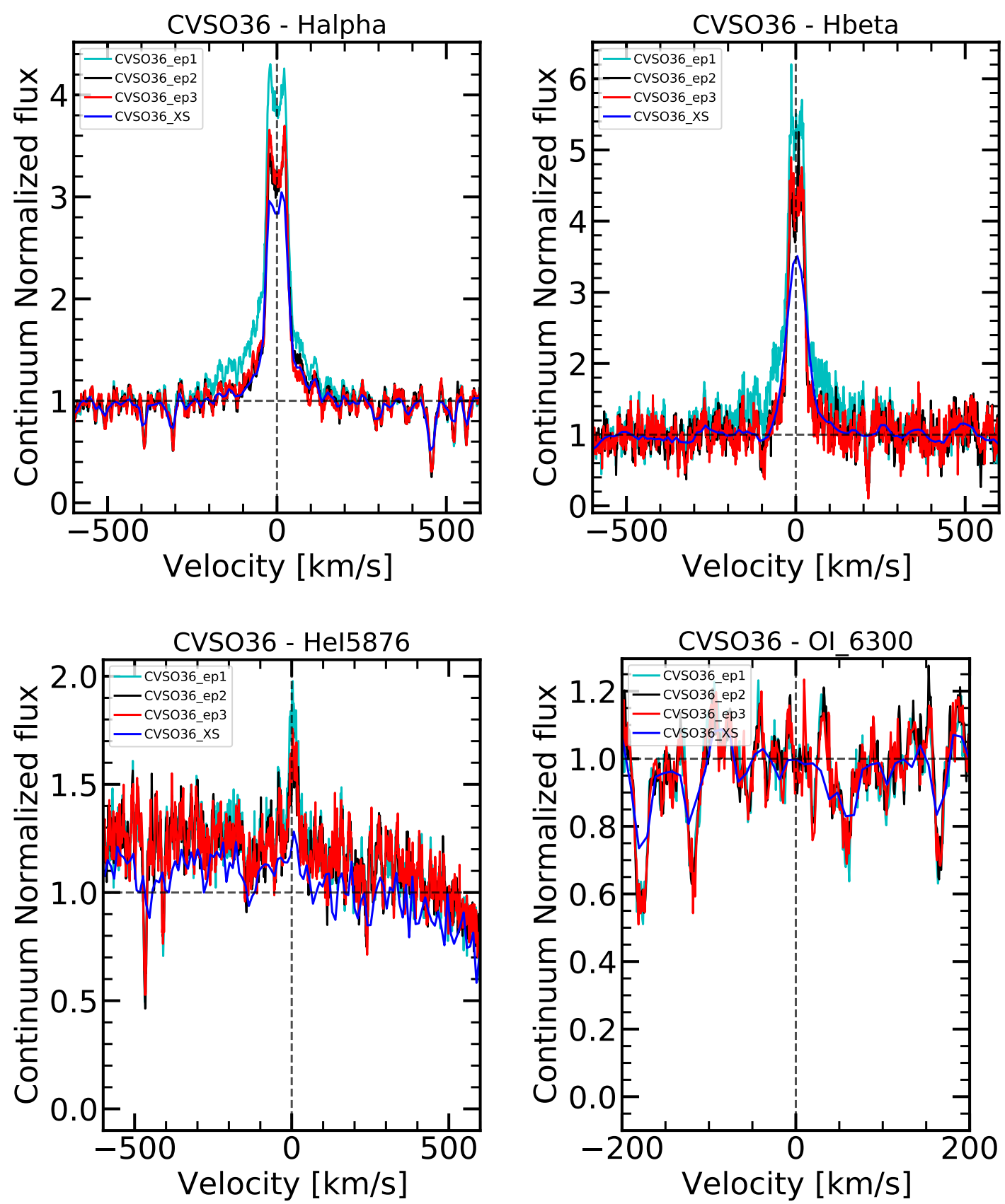

Fig. G.2. Emission lines of the target CVSO 36 observed with UVES and X-shooter. 

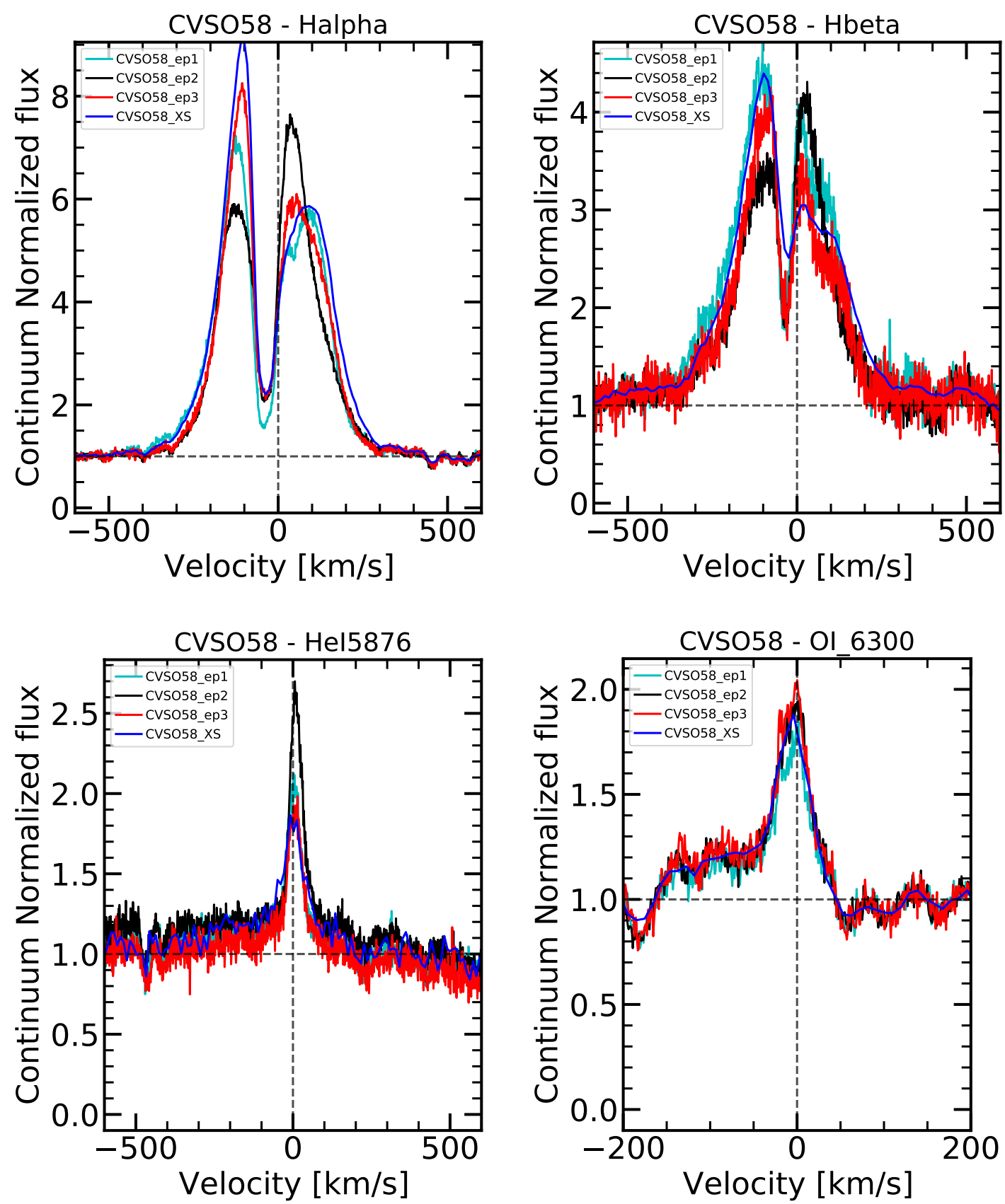

Fig. G.3. Emission lines of the target CVSO 58 observed with UVES and X-shooter. 

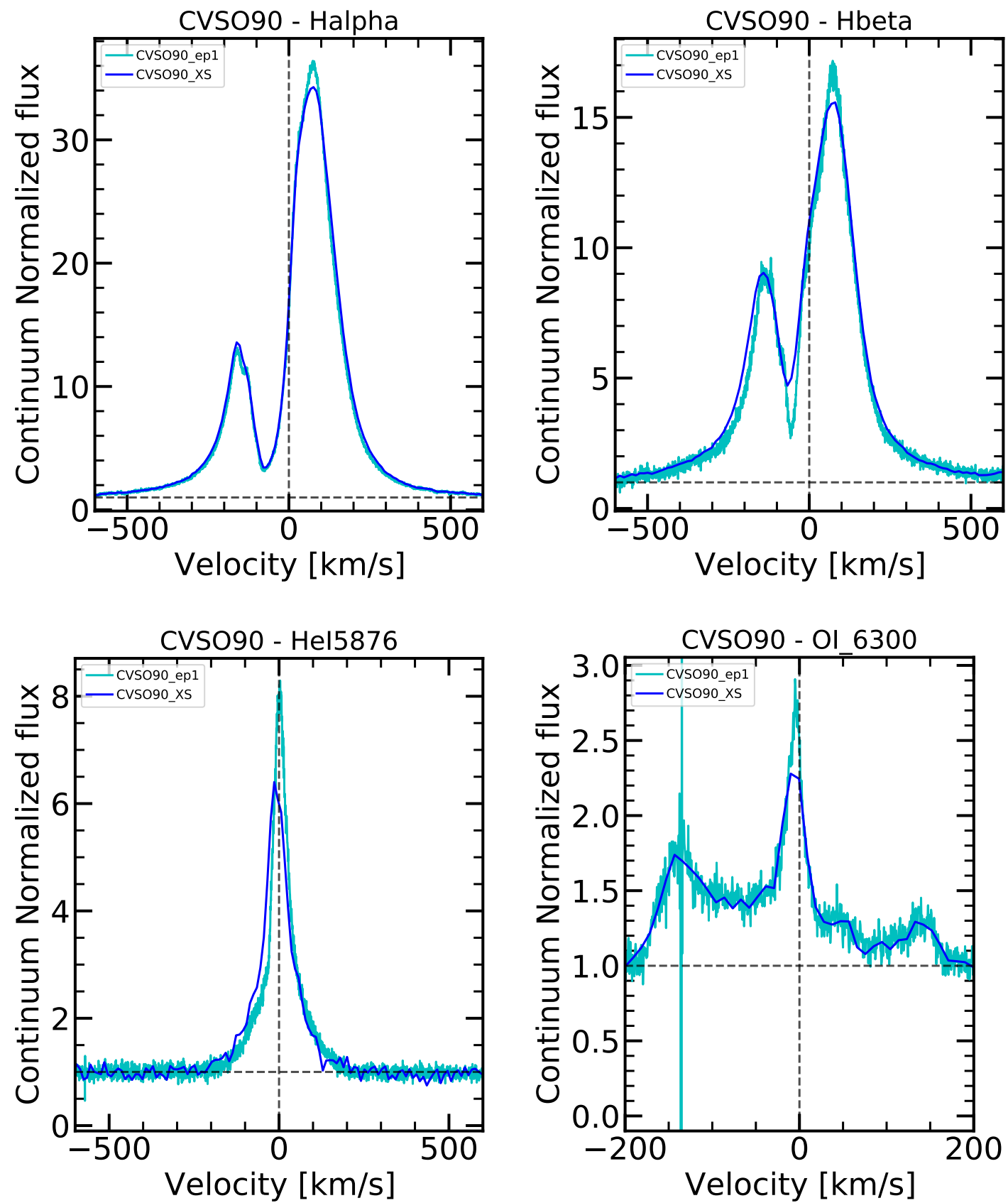

Fig. G.4. Emission lines of the target CVSO 90 observed with ESPRESSO and X-shooter. Only one epoch of ESPRESSO data was taken. 

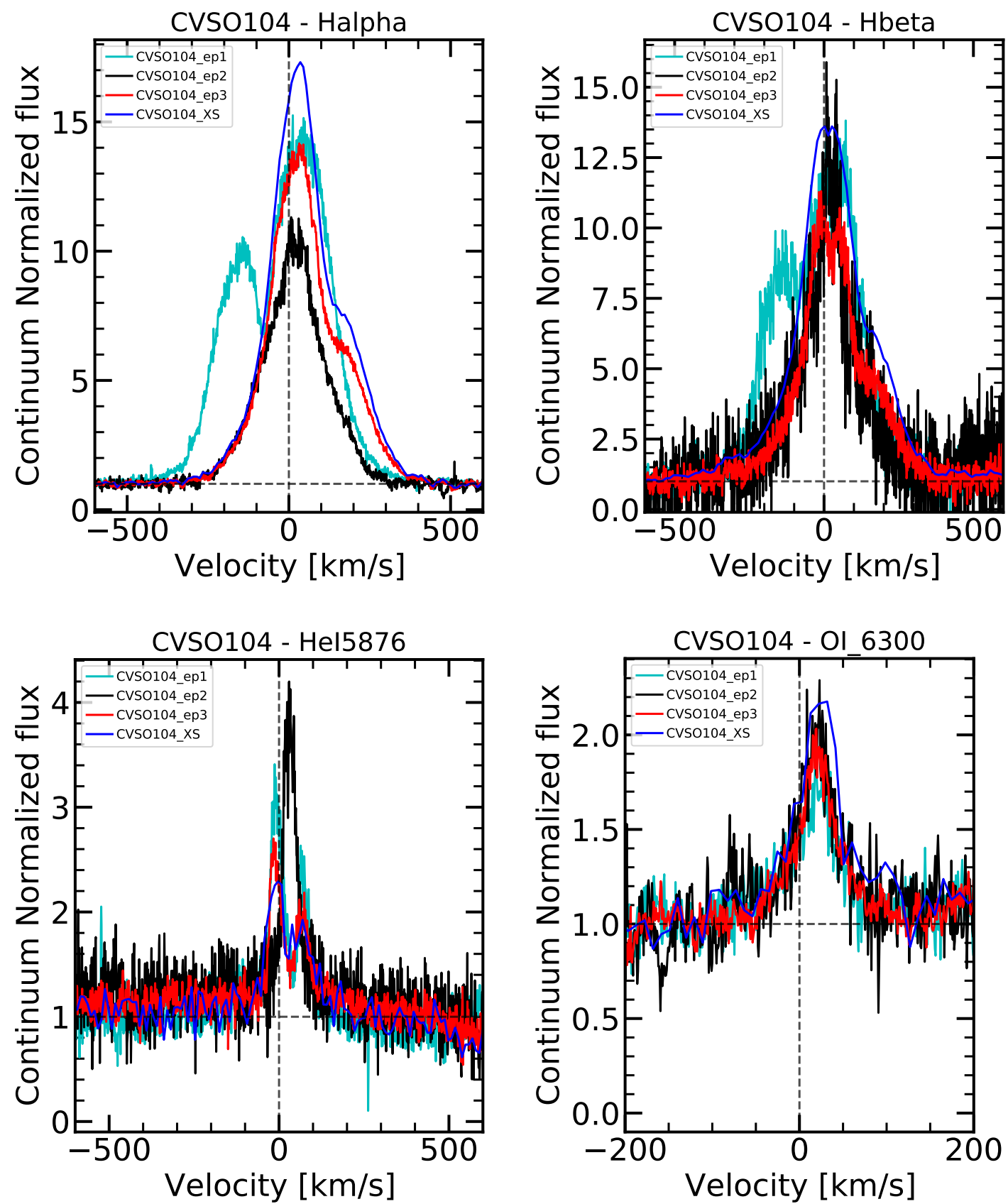

Fig. G.5. Emission lines of the target CVSO 104 observed with UVES and X-shooter. This target is a spectroscopic binary. 
C. F. Manara et al.: PENELLOPE. I. Survey presentation and Orion
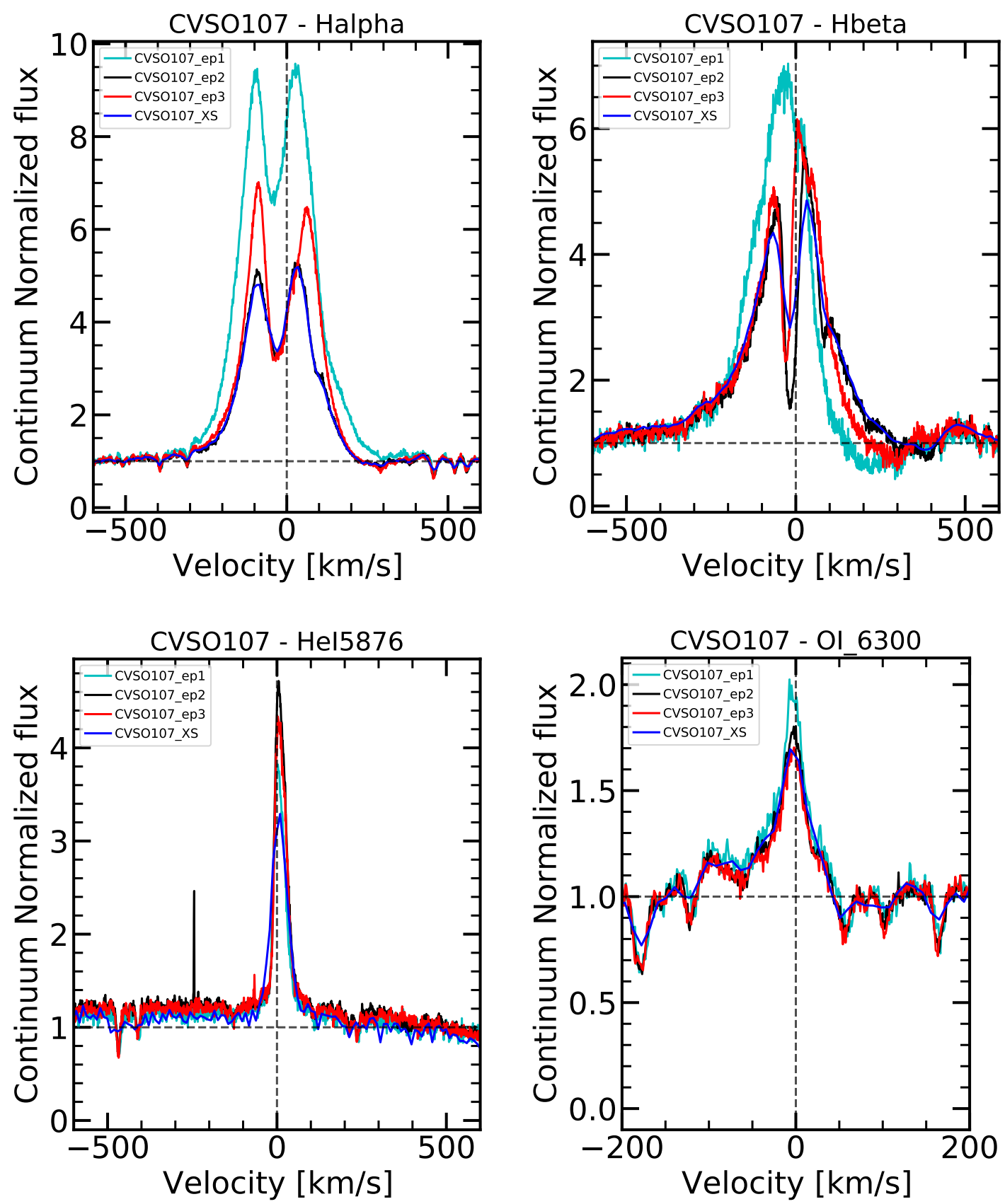

Fig. G.6. Emission lines of the target CVSO 107 observed with UVES and X-shooter. 

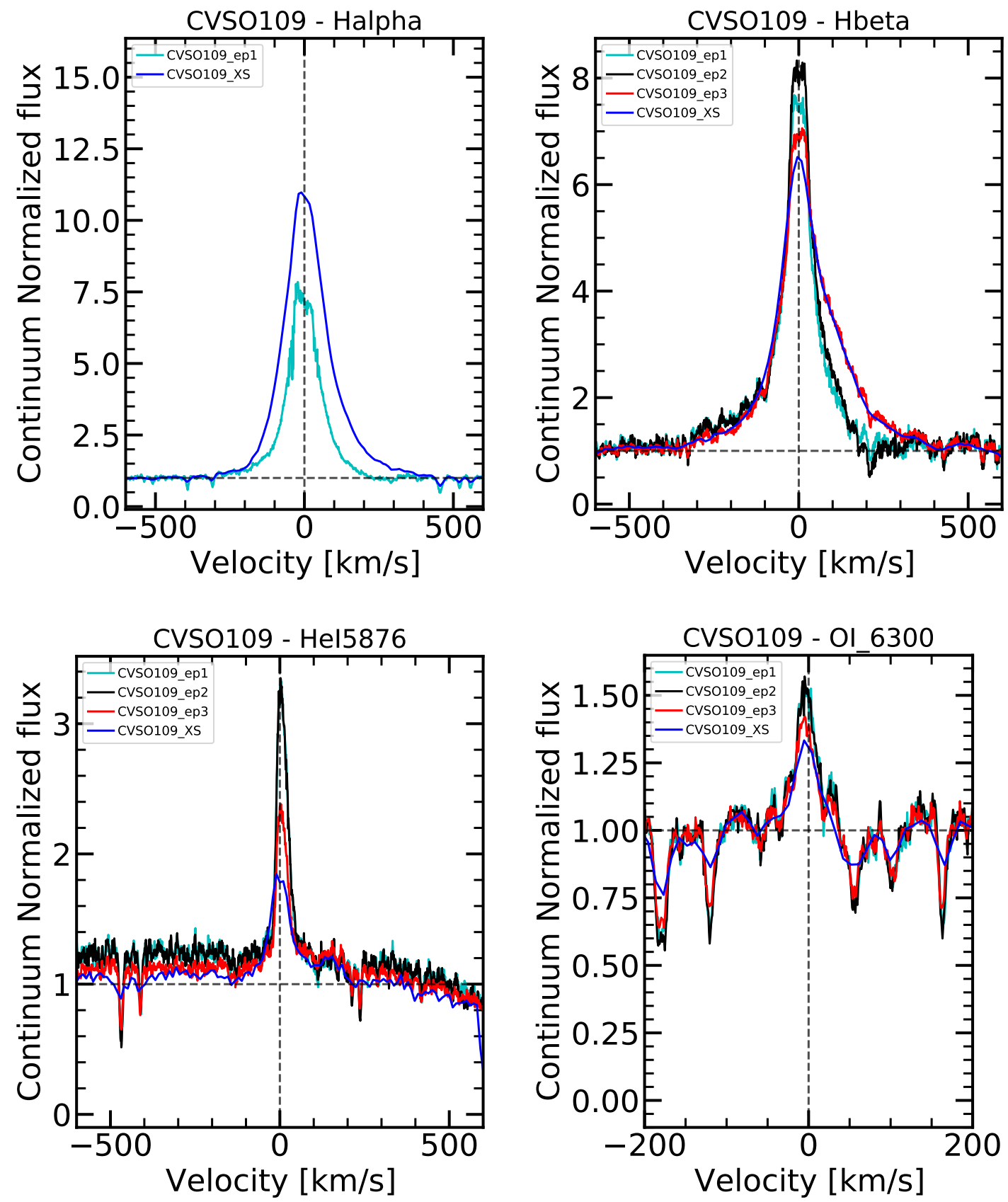

Fig. G.7. Emission lines of the target CVSO 109 observed with UVES and X-shooter. In the second and third epoch of UVES observations of CVSO109 the $\mathrm{H} \alpha$ line is saturated, and this is therefore not shown here. 

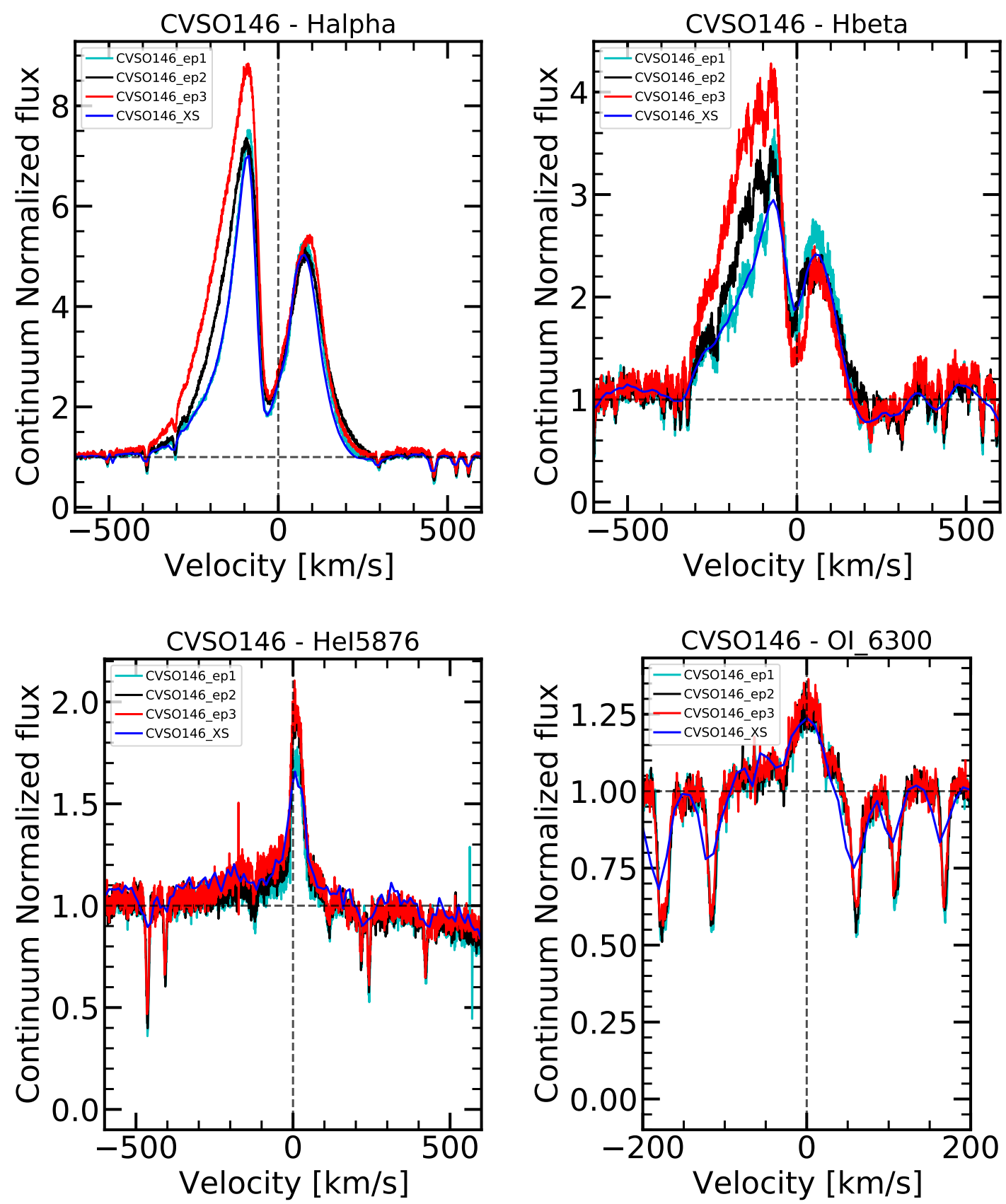

Fig. G.8. Emission lines of the target CVSO 146 observed with ESPRESSO and X-shooter. 

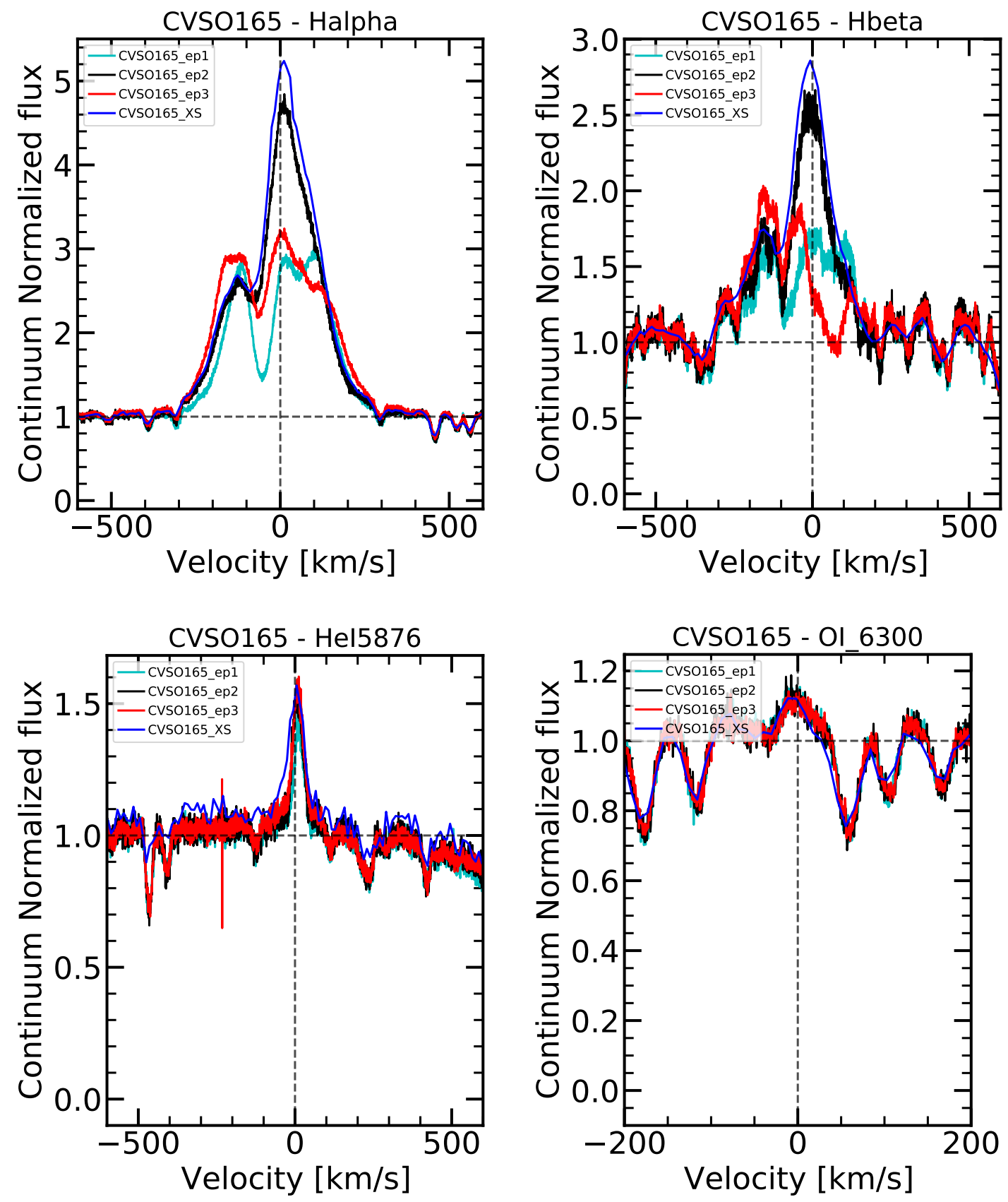

Fig. G.9. Emission lines of the target CVSO 165 observed with ESPRESSO and X-shooter. 

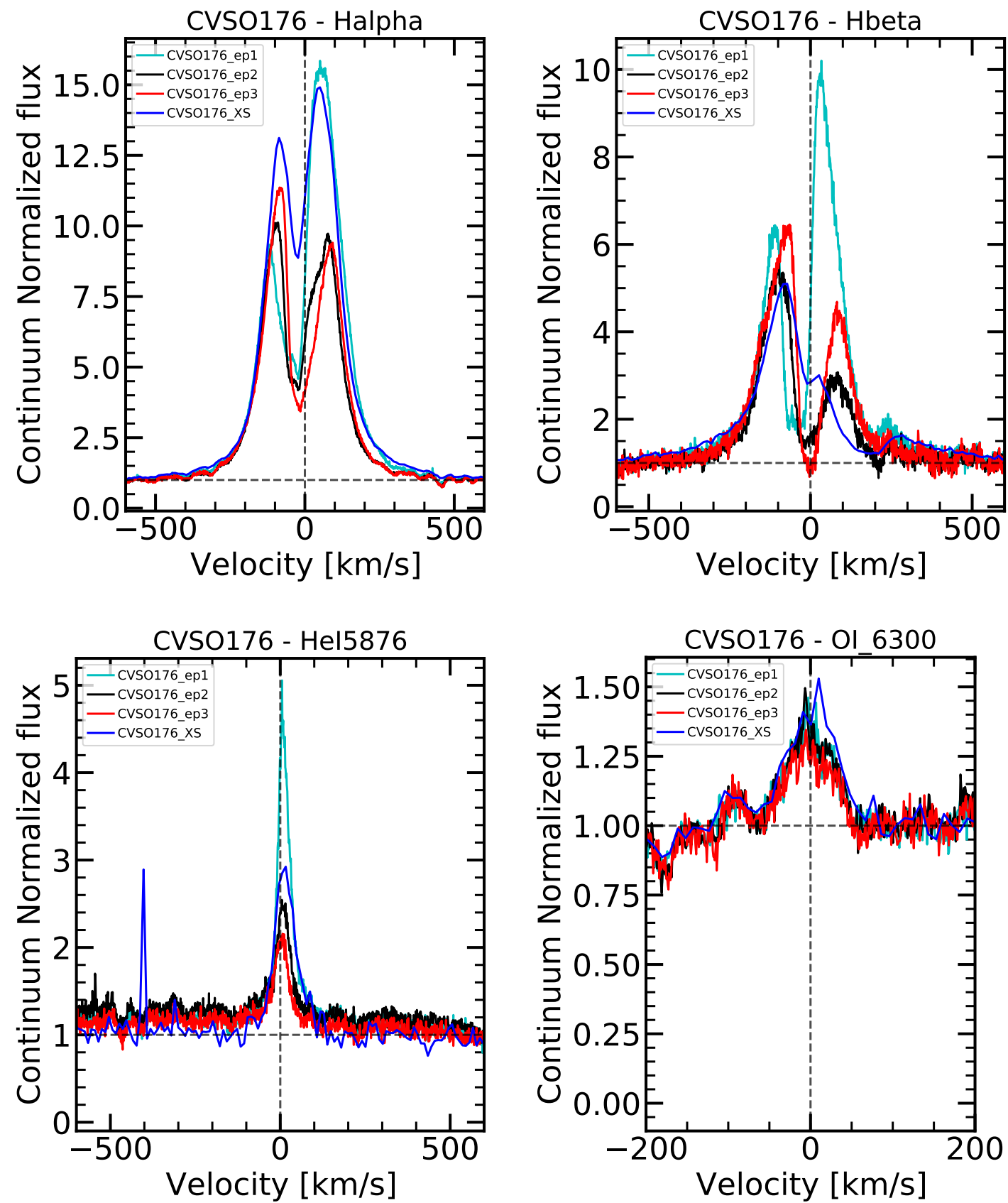

Fig. G.10. Emission lines of the target CVSO 176 observed with UVES and X-shooter. 

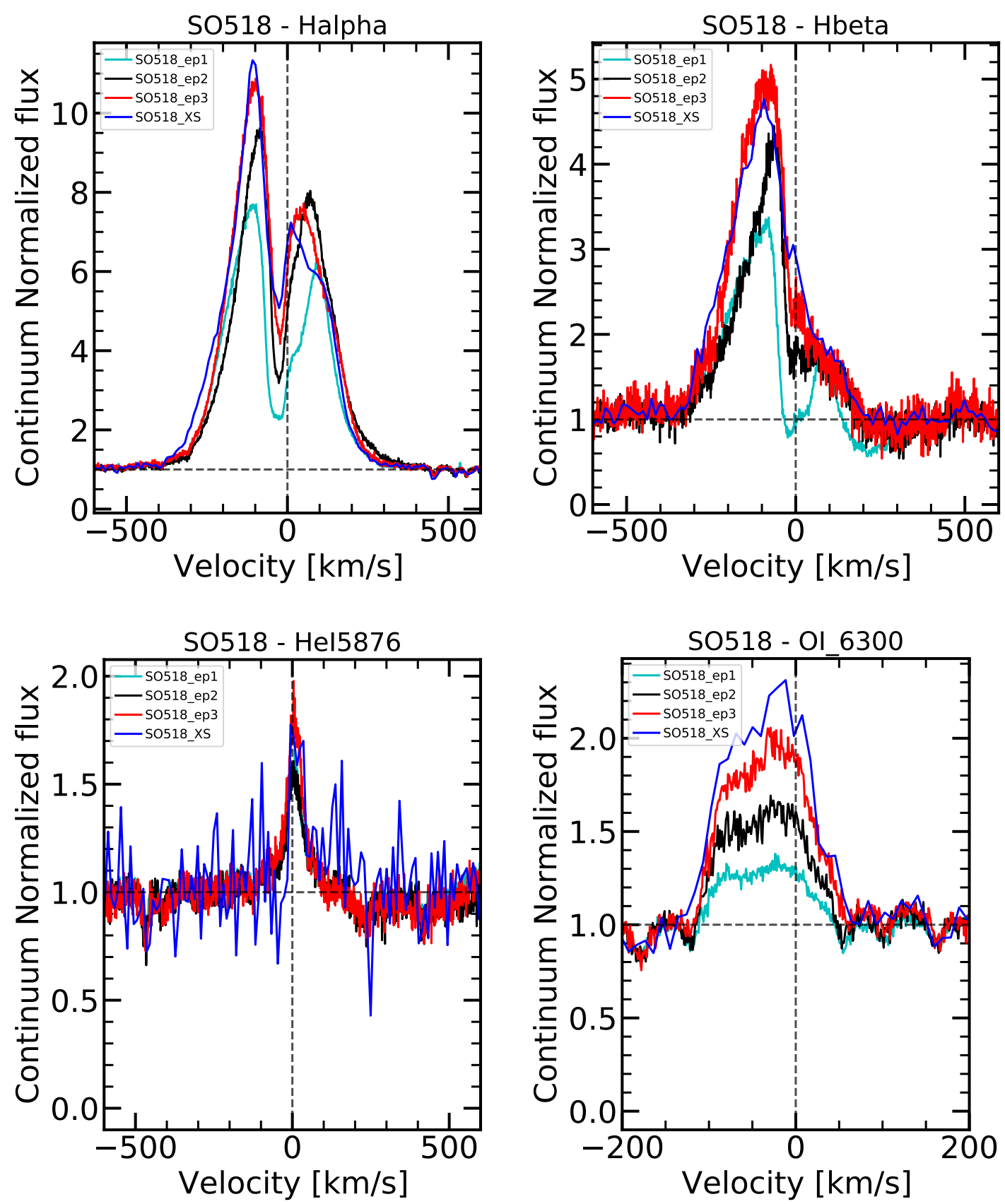

Fig. G.11. Emission lines of the target SO 518 observed with UVES and X-shooter. 

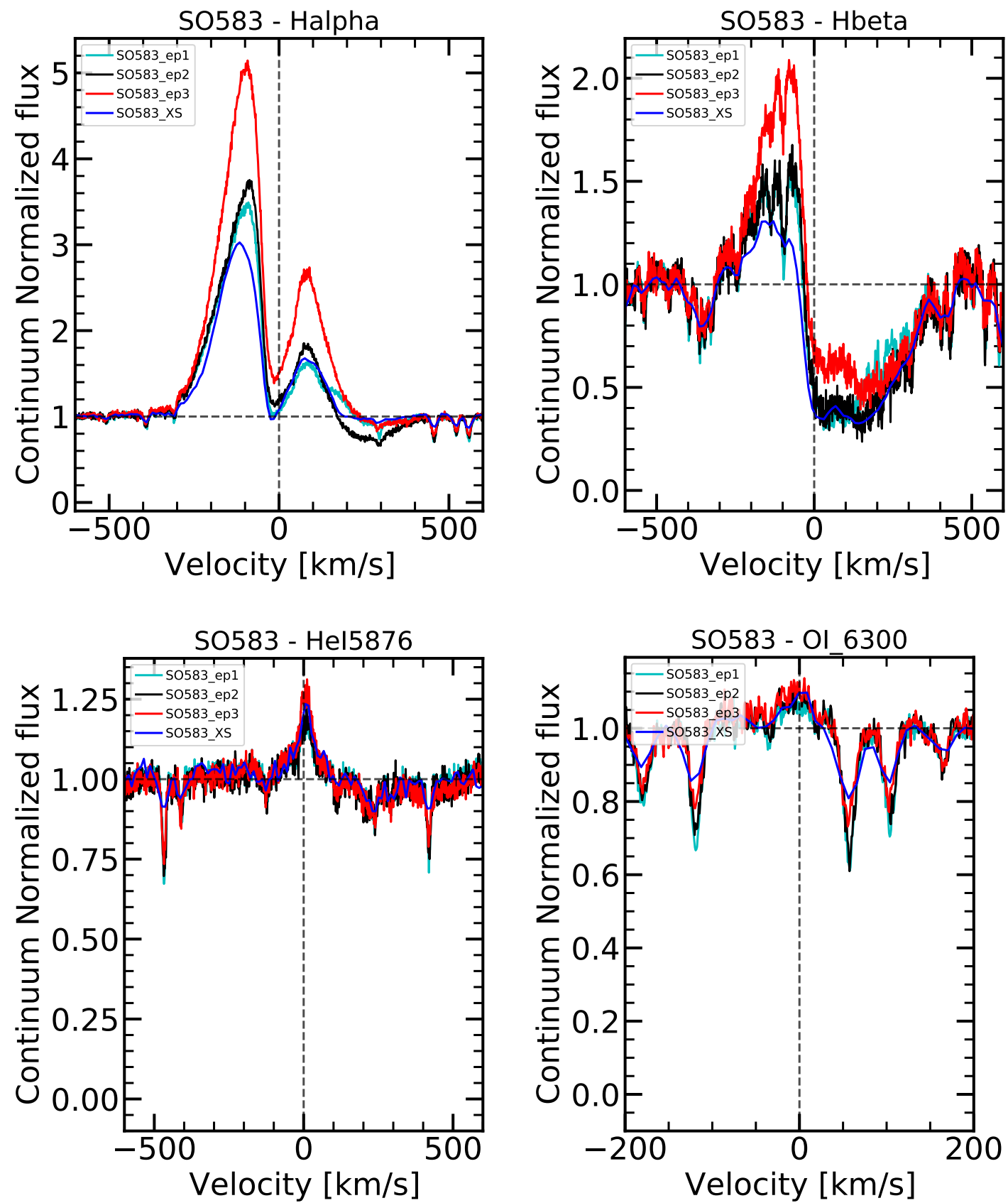

Fig. G.12. Emission lines of the target SO 583 observed with UVES and X-shooter. 

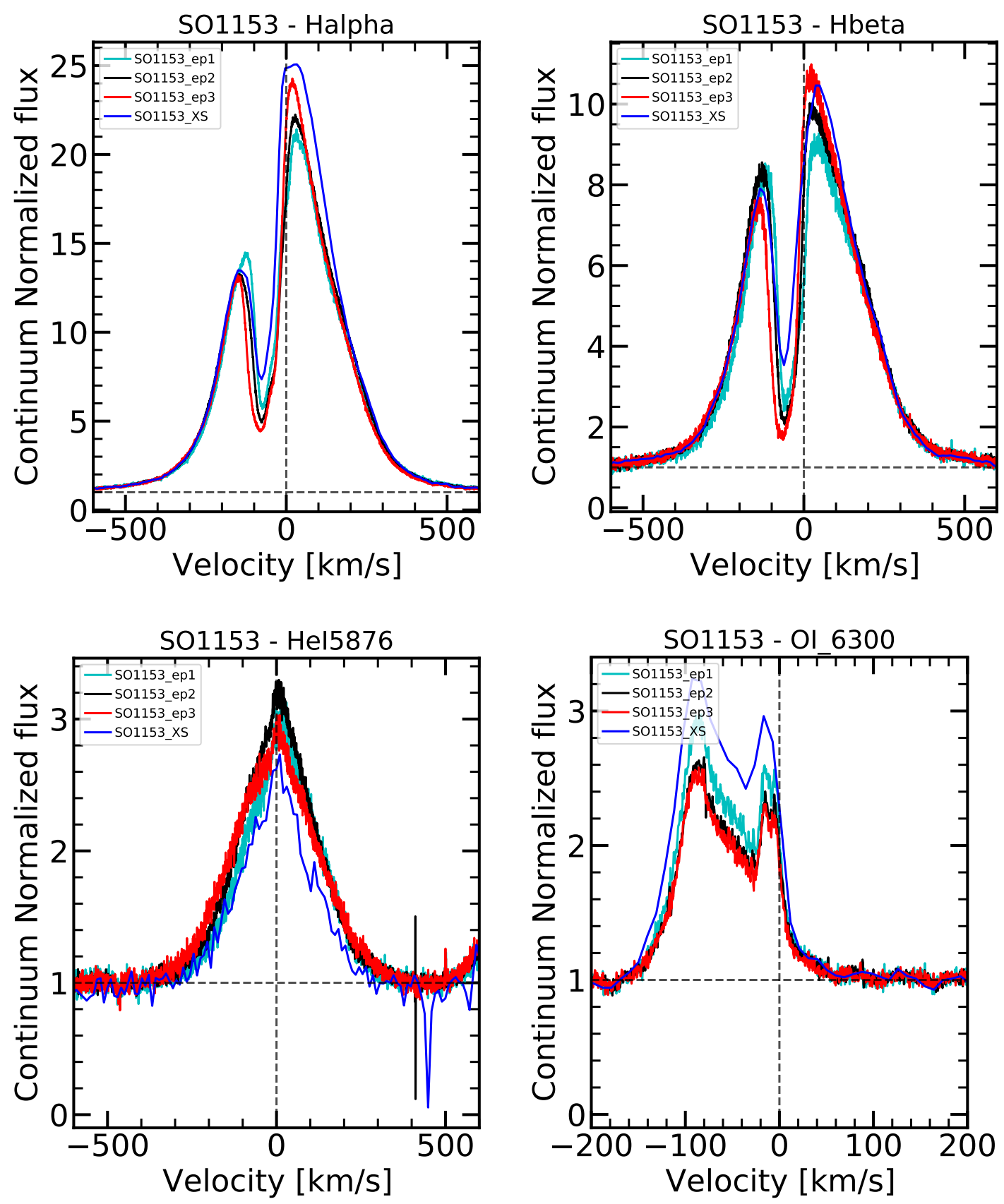

Fig. G.13. Emission lines of the target SO 1153 observed with UVES and X-shooter. 


\section{Appendix H: Examples of spectral subtraction with \\ ROTFIT}

Two examples of spectral subtraction for two stars with different mass accretion rate observed with UVES and ESPRESSO are shown in the five spectral regions in Fig. H.1.

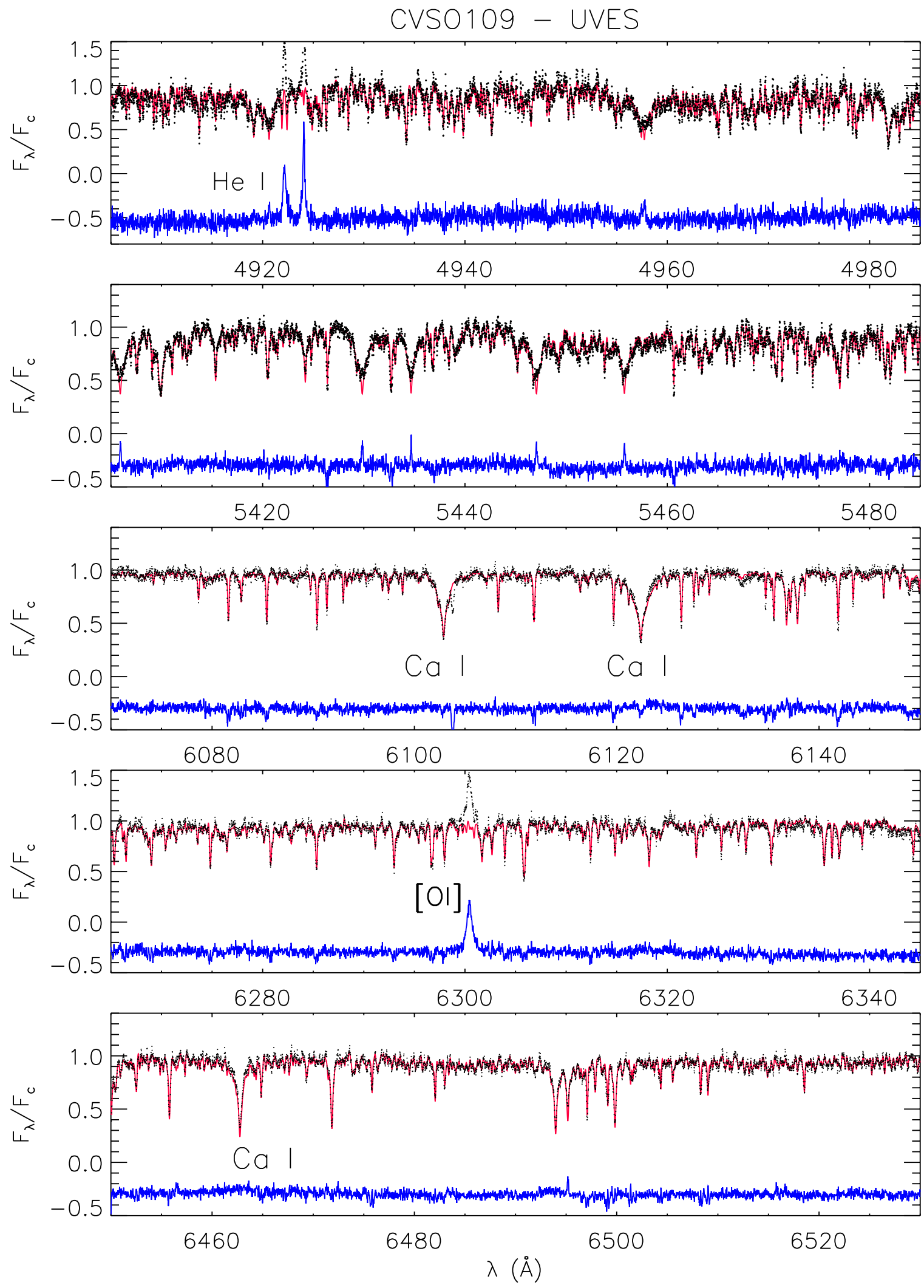

Fig. H.1. Subtraction of the non-active, lithium-poor template (red lines) from the spectrum of CVSO 109 (black dots) in the five different spectral regions. The most prominent emission and absorption lines have been indicated. 

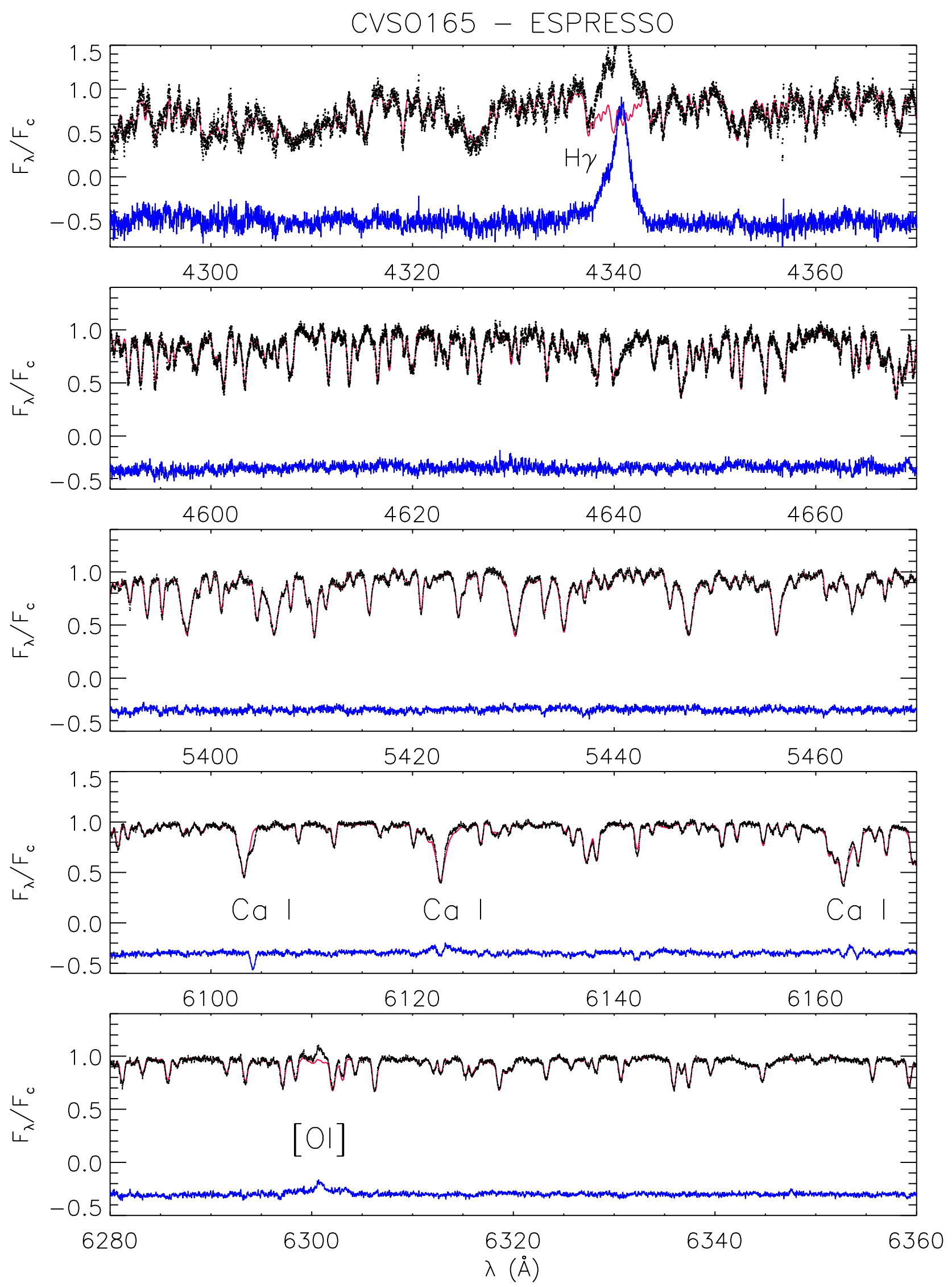

Fig. H.2. Subtraction of the non-active, lithium-poor template (red lines) from the spectrum of CVSO 165 (black dots) in five different spectral regions. The most prominent emission and absorption lines have been indicated. 